\title{
Approximately Counting Triangles in Sublinear Time
}

\author{
Talya Eden* Amit Levi ${ }^{\dagger} \quad$ Dana Ron ${ }^{\ddagger} \quad$ C. Seshadhri ${ }^{\S}$
}

\begin{abstract}
We consider the problem of estimating the number of triangles in a graph. This problem has been extensively studied in both theory and practice, but all existing algorithms read the entire graph. In this work we design a sublinear-time algorithm for approximating the number of triangles in a graph, where the algorithm is given query access to the graph. The allowed queries are degree queries, vertex-pair queries and neighbor queries.

We show that for any given approximation parameter $0<\epsilon<1$, the algorithm provides an estimate $\widehat{t}$ such that with high constant probability, $(1-\epsilon) \cdot t<\widehat{t}<(1+\epsilon) \cdot t$, where $t$ is the number of triangles in the graph $G$. The expected query complexity of the algorithm is $\left(\frac{n}{t^{1 / 3}}+\min \left\{m, \frac{m^{3 / 2}}{t}\right\}\right) \cdot \operatorname{poly}(\log n, 1 / \epsilon)$, where $n$ is the number of vertices in the graph and $m$ is the number of edges, and the expected running time is $\left(\frac{n}{t^{1 / 3}}+\frac{m^{3 / 2}}{t}\right) \cdot \operatorname{poly}(\log n, 1 / \epsilon)$. We also prove that $\Omega\left(\frac{n}{t^{1 / 3}}+\min \left\{m, \frac{m^{3 / 2}}{t}\right\}\right)$ queries are necessary, thus establishing that the query complexity of this algorithm is optimal up to polylogarithmic factors in $n$ (and the dependence on $1 / \epsilon)$.
\end{abstract}

${ }^{*}$ School of Computer Science, Tel Aviv University, talyaa01@gmail.com

${ }^{\dagger}$ School of Electrical Engineering, Tel Aviv University, amitlev3@post.tau.ac.il

${ }^{\ddagger}$ School of Electrical Engineering, Tel Aviv University, danaron@tau.ac.il. This research was partially supported by the Israel Science Foundation grant No. 671/13 and by a grant from the Blavatnik fund.

${ }^{\S}$ University of California, Santa Cruz, scomandu@ucsc.edu 


\section{Contents}

1 Introduction $\quad 2$

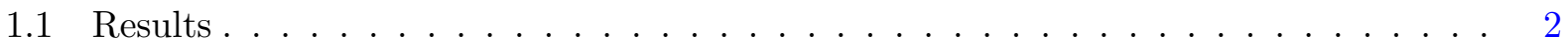

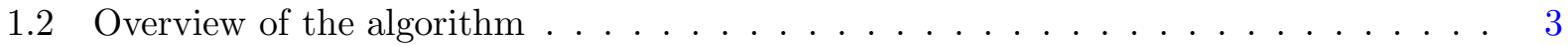

1.2.1 A simple oracle-based procedure for a $1 / 3$-estimate . . . . . . . . . . . . 3

1.2.2 Assigning weights to triangles so as to improve the estimate . . . . . . . . . 3

1.2.3 Deciding whether a vertex is heavy . . . . . . . . . . . . . . 3

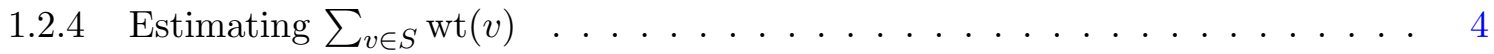

1.3 A high level discussion of the lower bound . . . . . . . . . . . . . . . 5

1.4 Related Work . . . . . . . . . . . . . . . . . . . . . . 5

1.4.1 Approximating graph parameters in sublinear time . . . . . . . . . . 5

1.4 .2 Triangle counting . . . . . . . . . . . . . . . 5

2 Preliminaries $\quad 6$

3 The Algorithm $\quad 7$

3.1 Heavy and light vertices . . . . . . . . . . . . . . . 7

3.2 A procedure for deciding whether a vertex is heavy . . . . . . . . . . . . 9

3.3 Estimating the number of triangles given $\bar{m}$ and $\bar{t} \ldots \ldots \ldots \ldots \ldots$

3.4 The final algorithm . . . . . . . . . . . . . . . . . . . . . 15

4 A Lower Bound $\quad 17$

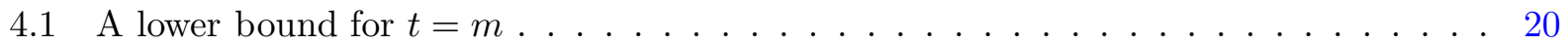

4.1 .1 The lower-bound construction . . . . . . . . . . . . . . 20

4.1.2 Definition of the processes $P_{1}$ and $P_{2} \ldots \ldots \ldots \ldots \ldots \ldots$

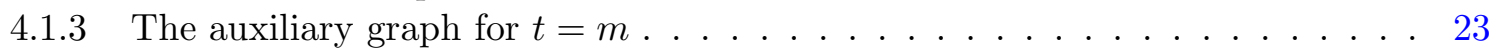

4.1 .4 Statistical distance . . . . . . . . . . . . . . . . . 24

4.2 A lower bound for $m<t<m^{3 / 2} \ldots \ldots \ldots \ldots \ldots \ldots$

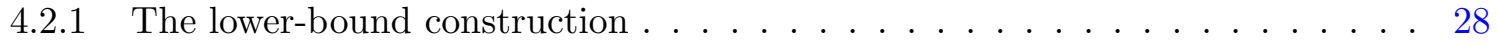

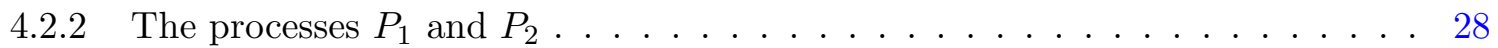

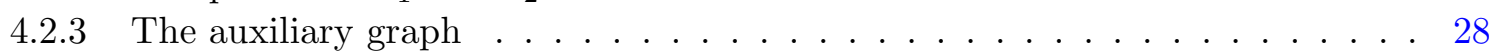

4.2 .4 Statistical distance . . . . . . . . . . . . . . . . . . . . . 29

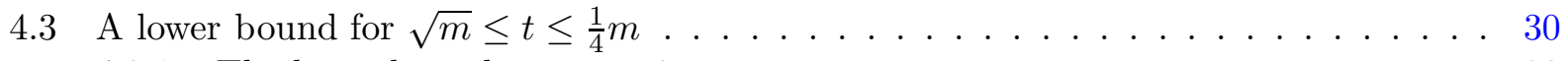

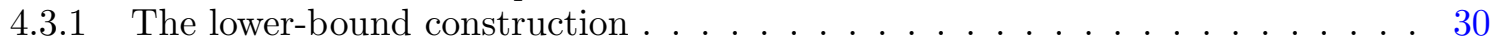

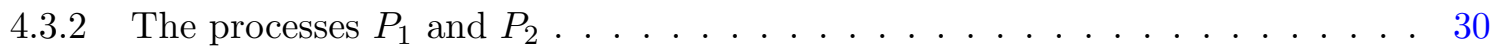

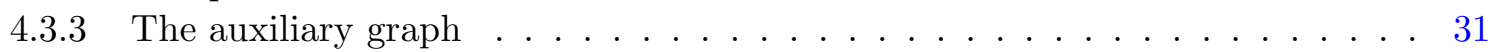

4.3 .4 Statistical distance . . . . . . . . . . . . . . . . . . . . 32

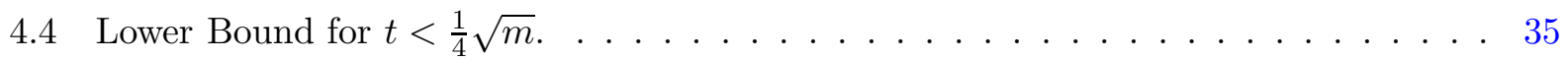

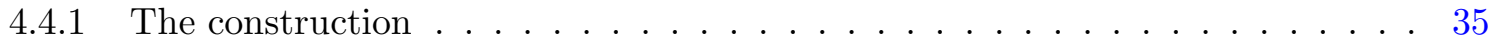

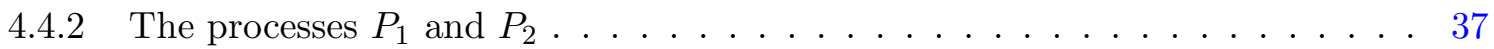

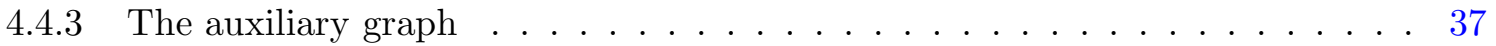

4.4 .4 Statistical distance . . . . . . . . . . . . . . . . . . . 38

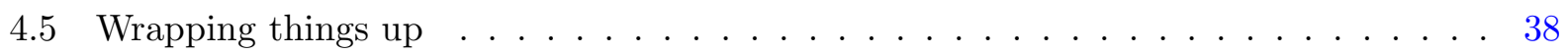




\section{Introduction}

Counting the number of triangles in a graph is a fundamental algorithmic problem. In the study of complex networks and massive real-world graphs, triangle counting is a key operation in graph analysis for bioinformatics, social networks, community analysis, and graph modeling [HL70, Col88, Por00, EM02, $\mathrm{MSOI}^{+}$02, Bur04, BBCG08, FWVDC10, BHLP11, SKP12]. In the theoretical computer science community, the primary tool for counting the number of triangles is fast matrix multiplication [IR78, AYZ97, BPWZ14]. On the more applied side, there is a plethora of provable and practical algorithms that employ clever sampling methods for approximate triangle counting [CN85, SW05b, SW05a, Tso08, TKMF09, Avr10, KMPT12, CC11, SV11, TKM11, AKM13, SPK13, TPT13]. Triangle counting has also been a popular problem in the streaming setting [BYKS02, JG05, BFL+06, AGM12, KMSS12, JSP13, PTTW13, TPT13, ADNK14].

All these algorithms read the entire graph, which may be time consuming when the graph is very large. In this work, we focus on sublinear algorithms for triangle counting. We assume the following query access to the graph, which is standard for sublinear algorithms that approximate graph parameters. The algorithm can make: (1) Degree queries, in which the algorithm can query the degree $d_{v}$ of any vertex $v$. (2) Neighbor queries, in which the algorithm can query what vertex is the $i^{\text {th }}$ neighbor of a vertex $v$, for any $i \leq d_{v}$. (3) Vertex-pair queries, in which the algorithm can query for any pair of vertices $v$ and $u$ whether $(u, v)$ is an edge.

Gonen et al. [GRS11], who studied the problem of approximating the number of stars in a graph in sublinear time, also considered the problem of approximating the number of triangles in sublinear time. They proved that there is no sublinear approximation algorithm for the number of triangles when the algorithm is allowed to perform degree and neighbor queries (but not pair queries). ${ }^{1}$

They asked whether a sublinear algorithm exists when allowed vertex-pair queries in addition to degree and neighbor queries. We show that this is indeed the case.

\section{$1.1 \quad$ Results}

Let $G$ be a graph with $n$ vertices, $m$ edges, and $t$ triangles. We describe an algorithm that, given an approximation parameter $0<\epsilon<1$ and query access to $G$, outputs an estimate $\widehat{t}$, such that with high constant probability (over the randomness of the algorithm), $(1-\epsilon) \cdot t \leq \widehat{t} \leq(1+\epsilon) \cdot t$. The expected query complexity of the algorithm is

$$
\left(\frac{n}{t^{1 / 3}}+\min \left\{m, \frac{m^{3 / 2}}{t}\right\}\right) \cdot \operatorname{poly}(\log n, 1 / \epsilon),
$$

and its expected running time is $\left(\frac{n}{t^{1 / 3}}+\frac{m^{3 / 2}}{t}\right) \cdot \operatorname{poly}(\log n, 1 / \epsilon)$. We show that this result is almost optimal by proving that the number of queries performed by any multiplicative-approximation algorithm for the number of triangles in a graph is

$$
\Omega\left(\frac{n}{t^{1 / 3}}+\min \left\{m, \frac{m^{3 / 2}}{t}\right\}\right) .
$$

\footnotetext{
${ }^{1}$ To be precise, they showed that there exist two families of graphs over $m=\Theta(n)$ edges, such that all graphs in one family have $\Theta(n)$ triangles, all graphs in the other family have no triangles, but in order to distinguish between a random graph in the first family and random graph in the second family, it is necessary to perform $\Omega(n)$ degree and neighbor queries.
} 


\subsection{Overview of the algorithm}

For the sake of clarity, we suppress any dependencies on the approximation parameter $\epsilon$ and on $\log n$ using the notation $O^{*}(\cdot)$.

\subsubsection{A simple oracle-based procedure for a 1/3-estimate}

First, let us assume access to an oracle that, given a vertex $v$, returns $t_{v}$, the number of triangles that $v$ is incident to. Note that $t=\sum_{v} t_{v} / 3$. An unbiased estimate is obtained by sampling, uniformly at random, a (multi-)set $S$ of $s$ vertices, and outputting $t_{S}=\frac{1}{3} \cdot \frac{n}{s} \cdot \sum_{v \in S} t_{v}$. Yet this estimate can have extremely large variance (consider the "wheel" graph where there is one vertex with $t_{v}=\Theta(n)$ and all other $t_{v}$ 's are constant). Inspired by work on estimating the average degree [Fei06, GR08], we can reduce the variance by simply "cutting off" the contribution of vertices $v$ for which $t_{v}$ is above a certain threshold. Call such vertices heavy, and denote the remaining light. If the threshold is set to $\Theta\left(t^{2 / 3} / \epsilon^{1 / 3}\right)$, then the number of heavy vertices is $O\left((\epsilon t)^{1 / 3}\right)$. This implies that the total number of triangles in which all three endpoints are heavy is $O(\epsilon t)$.

Hence, suppose we define $\widetilde{t}_{v}$ to be $t_{v}$ if $t_{v} \leq t^{2 / 3} / \epsilon^{1 / 3}$ and 0 otherwise, and consider $\widetilde{t}_{S}=$ $\frac{1}{3} \cdot \frac{n}{s} \cdot \sum_{v \in S} \widetilde{t}_{v}$. We can argue that $\mathbf{E}\left[\widetilde{t}_{S}\right] \in[(1 / 3-\epsilon) t, t]$, since (roughly speaking) every triangle that contains at least one light vertex is counted at least once. Since $\widetilde{t}_{v}$ ranges between 0 and $t^{2 / 3} / \epsilon^{1 / 3}$, by applying the multiplicative Chernoff bound, a sample of size $s=O^{*}\left(\frac{n}{t^{1 / 3}}\right)$ is sufficient to ensure that with high constant probability $\widetilde{t}_{S}$ is in the range $\left[\left(\frac{1}{3}-2 \epsilon\right) \cdot t,(1+\epsilon) \cdot t\right]$.

\subsubsection{Assigning weights to triangles so as to improve the estimate}

To improve the approximation, we assign weights to triangles inversely proportional to the number of their light endpoints (rather than assigning a uniform weight of $\frac{1}{3}$ as is done when defining $\left.\widetilde{t}_{S}=\frac{n}{s} \cdot \sum_{v \in S} \frac{1}{3} \cdot \widetilde{t}_{v}\right)$. If for each light vertex $v$ we let $\operatorname{wt}(v)$ be the sum over the weights of all triangles that $v$ participates in and for each heavy vertex $v$ we let $\operatorname{wt}(v)=\widetilde{t}_{v}=0$, then the expected value of $\frac{n}{s} \cdot \sum_{v \in S} \operatorname{wt}(v)$ is in $[(1-O(\epsilon)) \cdot t,(1+O(\epsilon)) \cdot t]$.

To get rid of the fictitious oracle, we must resolve two issues. The first issue is efficiently deciding whether a vertex is heavy or light, and the second is approximating $\frac{n}{s} \cdot \sum_{v \in S} \operatorname{wt}(v)$, assuming we have a procedure for deciding whether a vertex is heavy or light. We next discuss each of these two issues. For convenience, we will assume that the algorithm already has constant factor estimates for $m$ and $t$. This can be removed by approximating $m$ and performing a geometric search on $t$.

\subsubsection{Deciding whether a vertex is heavy}

Let $v$ be a fixed vertex with degree $d_{v}$. Consider an edge $e$ incident to $v$, and let $u$ be the other endpoint of this edge. Let $t_{e}$ denote the number of triangles that $e$ belongs to. Consider the random variable $Y$ defined by selecting, uniformly at random, a neighbor $w$ of $u$, and setting $Y=d_{u}$ if $(v, w)$ is an edge (so that $(v, u, w)$ is a triangle) and $Y=0$ otherwise. Since the number of neighbors of $u$ that form a triangle with $v$ is $t_{e}$, the expected value of $Y$ is $\frac{t_{e}}{d_{u}} \cdot d_{u}=t_{e}$. Now consider selecting (uniformly at random) several edges incident to $v$, denoted $e_{1}, \ldots, e_{r}$, and for each edge $e_{j}$ selected, defining the corresponding random variable $Y_{j}$. Then the expected value of $\frac{1}{r} \sum_{j=1}^{r} Y_{j}$

is $\frac{1}{d_{v}} \cdot \sum_{e=(v, u)} t_{e}=\frac{2}{d_{v}} \cdot t_{v}$. If we multiply by $d_{v} / 2$, then we get an unbiased estimator for $t_{v}$, which in particular can indicate whether $v$ is heavy or light.

However, once again the difficulty is with the variance of this estimator and the implication on the complexity of the resulting decision procedure. To address these difficulties we modify 
the procedure described above as follows. First, if $d_{v}$ is above a certain threshold, then $v$ is also considered heavy (where this threshold is of order $O\left(m /(\epsilon t)^{1 / 3}\right)$, so that the total number of triangles in which all three endpoints are heavy remains $O(\epsilon t)$. Second, observe that when trying to estimate the number of triangles that an edge $e_{j}=\left(v, x_{j}\right)$ participates in, we can either select a random neighbor $w$ of $v$ and check whether $\left(x_{j}, w\right) \in E$, or we can select a random neighbor $w$ of $x_{j}$ and check whether $(v, w) \in E$. Since it is advantageous for the sake of the complexity to consider the endpoint that has a smaller degree, we do the following. Each time we select an edge $e_{j}=\left(v, x_{j}\right)$ incident to $v$, we let $u_{j}$ be the endpoint of $e_{j}$ that has smaller degree. If $d_{u_{j}}$ is relatively large (larger than $\sqrt{m}$ ), then we select $k=\left\lceil d_{u_{j}} / \sqrt{m}\right\rceil$ neighbors of $u_{j}$ and let $Y_{j}$ equal $d_{u_{j}}$ times the fraction among these neighbors that close a triangle with $e_{j}$. The setting of $k$ implies a bound on the variance of $Y_{j}$ (conditioned on the choice of $e_{j}$ ), which is $\sqrt{m}$ times its expected value, $t_{e_{j}}$. Third, in order to bound the variance due to the random choice of edges $e_{j}$ incident to $v$, we do the following. We assign each triangle that $v$ participates in to a unique edge incident to $v$ and modify the definition of $t_{e}$ to be the number of such triangles that are assigned to $e$. The assignment is such that $t_{e}$ is always upper bounded by $O(\sqrt{m})$. Finally, we perform a standard median selection over $O(\log n)$ repetitions of the procedure.

Our analysis shows that it suffices to set $r$ (the number of random edges incident to $v$ that are selected) to be $O^{*}\left(\frac{m^{3 / 2}}{t}\right)$ so as to ensure the correctness of the procedure (with high probability). In the analysis of the expected query complexity and running time of the procedure we have to take into account the number of iterations $k=\left\lceil d_{u_{j}} / \sqrt{m}\right\rceil$ for each selected (lower degree endpoint) $u_{j}$ and argue that for every vertex $v$, the expected number of these iterations is a constant.

\subsubsection{Estimating $\sum_{v \in S} \mathbf{w t}(v)$}

Suppose we have a (multi-)set $S$ of vertices such that $\frac{n}{s} \cdot \sum_{v \in S} \operatorname{wt}(v)$ is indeed in $[(1-O(\epsilon))$. $t,(1+O(\epsilon)) \cdot t]$ (which we know occurs with high probability if we select $s=O^{*}\left(\frac{n}{t^{1 / 3}}\right)$ vertices uniformly at random). Consider the set of edges incident to vertices in $S$, where we view edges as directed, so that if there is an edge between $v$ and $v^{\prime}$ that both belong to $S$, then $\left(v, v^{\prime}\right)$ and $\left(v^{\prime}, v\right)$ are considered as two different edges. We denote this set of edges by $E_{S}$, and their number by $d_{S}$, where $d_{S}=\sum_{v \in S} d_{v}$. Suppose that for each edge $e=(v, x)$ we assign a weight $\operatorname{wt}(e)$, which is the sum of the weights of all triangles that $v$ participates in and are assigned to $e$ (where the weight of a triangle is as defined previously based on the number of light endpoints that it has). Then $\sum_{e \in E_{S}} \operatorname{wt}(e)=\sum_{v \in S} \operatorname{wt}(v)$.

The next idea is to sample edges in $E_{S}$ uniformly at random, and for each selected edge $e=(v, u)$ to estimate $\mathrm{wt}(e)$. An important observation is that since we can query the degrees of all vertices in $S$, we can efficiently select uniform random edges in $E_{S}$ (as opposed to the more difficult task of selecting random edges from the entire graph). Similarly to what was described in the decision procedure for heavy vertices, given an edge $e \in E_{S}$ we let $u$ be its endpoint that has smaller degree. We then select $\left\lceil\sqrt{m} / d_{u}\right\rceil$ random neighbors of $u$ and for each check whether it closes a triangle with $e$. For each triangle found that is assigned to $e$, we check how many heavy endpoints it has (using the aforementioned procedure for detecting heavy vertices) so as to compute the weight of the triangle. In this manner we can obtain random variables whose expected value is $\frac{1}{d_{S}} \sum_{v \in S} \operatorname{wt}(v)$, and whose variance is not too large (upper bounded by $\sqrt{m}$ times this expected value). We can now take an average over sufficiently many $\left(O^{*}\left(\frac{m^{3 / 2}}{t}\right)\right)$ such random variables and multiply by $d_{S} \cdot n$. By upper bounding the probability that $d_{S}$ is much larger than its expected value we can prove that the output of the algorithm is as desired. The expected query complexity and running 
time of the algorithm are shown to be $O^{*}\left(\frac{n}{t^{1 / 3}}+\frac{m^{3 / 2}}{t}\right)$.

Finally we note that if $t<m^{1 / 2}$ so that $\frac{m^{3 / 2}}{t}>m$, then we can replace $\frac{m^{3 / 2}}{t}$ with $m$ in the upper bound on the query complexity since we can store all queried edges so that no edge needs to be queried more than twice (once from each endpoint).

\subsection{A high level discussion of the lower bound}

Proving that every multiplicative-approximation algorithm must perform $\Omega\left(\frac{n}{t^{1 / 3}}\right)$ queries is fairly straightforward, and our main focus is on proving that $\Omega\left(\min \left\{m, \frac{m^{3 / 2}}{t}\right\}\right)$ queries are necessary as well. In order to prove this claim we define, for every $n$, every $1 \leq m \leq\left(\begin{array}{l}n \\ 2\end{array}\right)$ and every $1 \leq t \leq \min \left\{\left(\begin{array}{l}n \\ 3\end{array}\right), m^{3 / 2}\right\}$, a graph $G_{1}$ and a family of graphs $\mathcal{G}_{2}$ for which the following holds: (1) The graph $G_{1}$ and all the graphs in $\mathcal{G}_{2}$ have $n$ vertices and $m$ edges. (2) In $G_{1}$ there are no triangles, while the number of triangles in each graph $G \in \mathcal{G}_{2}$ is $\Theta(t)$. We prove that for values of $t$ such that $t \geq \sqrt{m}$, at least $\Omega\left(\frac{m^{3 / 2}}{t}\right)$ queries are required in order to distinguish with high constant probability between $G_{1}$ and a random graph in $\mathcal{G}_{2}$. We then prove that for values of $t$ such that $t<\sqrt{m}$, at least $\Omega(m)$ queries are required for this task. We give three different constructions for $G_{1}$ and $\mathcal{G}_{2}$ depending on the value of $t$ as a function of $m$ (where two of the constructions are for subcases of the case that $t \geq \sqrt{m}$ ). For further discussion of the lower bound, see Section 4 .

\subsection{Related Work}

\subsubsection{Approximating graph parameters in sublinear time}

We build on previous work on approximating the average degree of a graph and the number of stars [Fei06, GR08, GRS11]. Feige [Fei06] investigated the problem of estimating the average degree of a graph, denoted $\bar{d}$, when given query access to the degrees of the vertices. By performing a careful variance analysis, Feige proved that $O(\sqrt{n / \bar{d}} / \epsilon)$ queries are sufficient in order to obtain a $\left(\frac{1}{2}-\epsilon\right)$-approximation of $\bar{d}$. He also proved that a better approximation ratio cannot be achieved in sublinear time using only degree queries. The same problem was considered by Goldreich and Ron [GR08]. Goldreich and Ron proved that a $(1+\epsilon)$-approximation can be achieved with $O(\sqrt{n / \sqrt{\bar{d}}}) \cdot \operatorname{poly}(\log n, 1 / \epsilon)$ queries, if neighbor queries are also allowed.

Building on these ideas, Gonen et al. [GRS11] considered the problem of approximating the number of $s$-stars in a graph. Their algorithm only used neighbor and degree queries. A major difference between stars and triangles is that the former are non-induced subgraphs, while the latter are. Additional work on sublinear algorithms for estimating other graph parameters include those for approximating the size of the minimum weight spanning tree [CRT05, CS09, $\mathrm{CEF}^{+} 05$ ], maximum matching [NO08, YYI09] and of the minimum vertex cover [PR07, NO08, MR09, YYI09, HKNO09, ORRR12].

\subsubsection{Triangle counting}

Triangle counting has a rich history. A classic result of Itai and Rodeh showed that triangles can be enumerated in $O\left(\mathrm{~m}^{3 / 2}\right)$ time, and a more elegant algorithm was given by Chiba and Nishizeki [CN85]. The connections to matrix multiplication have been exploited for faster theoretical algorithms [IR78, AYZ97, BPWZ14]. In practice, there is a diverse body on work on 
counting triangles using different techniques, for different models. There are serial algorithms based on eigenvalue methods [Tso08, Avr10], graph sparsification [TDM+09, KMPT12, TKM11, PT12], and sampling paths [SW05b, SPK13]. Triangle counters have been given for MapReduce [Coh09, SV11, $\mathrm{KPP}^{+} 13$ ]; external memory models [CC11]; distributed settings [AKM13]; semi-streaming models [BBCG08, KMPT12]; one-pass streaming [BYKS02, JG05, BFL ${ }^{+}$06, AGM12, KMSS12, JSP13, PTTW13, TPT13, ADNK14]. It is worth noting that across the board, all these algorithms required reading the entire graph.

Most relevant to our work are various sampling algorithms, that set up a random variable whose expectation is directly related to the triangle count [SW05b, KMPT12, JG05, BFL ${ }^{+} 06$, SPK13, JSP13, PTTW13, TPT13, ADNK14]. Typically, this involves sampling some set of vertices or edges to get a set of three vertices. The algorithm checks whether the sampled set induces a triangle, and uses the probability of success to estimate the triangle count. We follow the basic same philosophy. But it is significantly more challenging to set up the "right" random experiment, since we cannot read the entire graph.

\section{Preliminaries}

Let $G=(V, E)$ be a simple graph with $|V|=n$ vertices and $|E|=m$ edges. For a vertex $v \in V$, we denote by $d_{v}$ the degree of the vertex, by $\Gamma_{v}$ the set of $v$ 's neighbors, and by $E_{v}$ the set of edges incident to $v$. We denote by $T_{v}$ the set of triangles incident to the vertex $v$, and let $t_{v}=\left|T_{v}\right|$. Similarly, the set of triangles in the graph $G$ is denoted by $T$, and the number of triangles in the graph in denote by $t$. We use $c, c_{1}, \ldots$ to denote sufficiently large constants.

We consider algorithms that can sample uniformly in $V$ and perform three types of queries:

1. Degree queries, in which the algorithm may query for the degree $d_{v}$ of any vertex $v$ of its choice.

2. Neighbor queries, in which the algorithm may query for the $i^{\text {th }}$ neighbor of any vertex $v$ of its choice. If $i>d_{v}$, then a special symbol (e.g. †) is returned. No assumption is made on the order of the neighbors of any vertex.

3. Pair queries, in which the algorithm may ask if there is an edge $(u, v) \in E$ between any pair of vertices $u$ and $v$.

We sometimes use set notations for operations on multisets. We use the notation $O^{*}(\cdot)$ to suppress dependencies on the approximation parameter $\epsilon$ or on $\log n$.

We use the following variant of the multiplicative Chernoff bound. Let $\chi_{1}, \ldots, \chi_{r}$ be $r$ independent random variables, such that $\chi_{i} \in[0, B]$ for some $B>0$ and $\mathbf{E}\left[\chi_{i}\right]=b$ for every $1 \leq i \leq r$. For every $\gamma \in(0,1]$ the following holds:

$$
\operatorname{Pr}\left[\frac{1}{r} \sum_{i=1}^{r} \chi_{i}>(1+\gamma) b\right]<\exp \left(-\frac{\gamma^{2} \cdot b \cdot r}{3 B}\right),
$$

and

$$
\operatorname{Pr}\left[\frac{1}{r} \sum_{i=1}^{r} \chi_{i}<(1-\gamma) b\right]<\exp \left(-\frac{\gamma^{2} \cdot b \cdot r}{2 B}\right) .
$$

We will also make an extensive use of Chebyshev's inequality: For a random variable $X$ and for $\gamma>0$

$$
\operatorname{Pr}[|X-\mathbf{E}[X]| \geq \gamma] \leq \frac{\operatorname{Var}[X]}{\gamma^{2}}
$$


We fix a total order on vertices denoted by $\prec$ as follows: $u \prec v$ if $d_{u}<d_{v}$ or $d_{u}=d_{v}$ and $u<v$ (in terms of id number). Given $u$ and $v$, two degree queries suffice to decide their ordering.

Claim 1. Fix any vertex $v$. The number of neighbors $w$ of $v$ such that $v \prec w$ is at most $\sqrt{2 m}$.

Proof. Let $S=\left\{w \mid w \in \Gamma_{v}, v \prec w\right\}$. Naturally, $d_{v} \geq|S|$. By definition of $\prec, \forall w \in S, d_{w} \geq d_{v} \geq|S|$. Thus, $\sum_{w \in S} d_{w} \geq|S|^{2}$ and $|S| \leq \sqrt{2 m}$.

\section{The Algorithm}

We start by introducing the notions of heavy and light vertices and how they can be utilized in the context of estimating the number of triangles. We then give a procedure for deciding (approximately) whether a vertex is heavy or light. Using this procedure we give an algorithm for estimating the number of triangles based on the following assumption (which is later removed).

Assumption 1. Our initial algorithm takes as input estimates $\bar{t}$ and $\bar{m}$ on the number of edges and triangles in the graph respectively, such that

1. $t / 4 \leq \bar{t} \leq t$.

2. $m / 6 \leq \bar{m}$.

Assumption 1 can be easily removed by performing a geometric search on $t$ and using the algorithm from [Fei06] to approximate $m$, as explained precisely in the proof of Theorem 13 .

For every vertex $v$, we view the set of edges $E_{v}$ as directed edges originating from $v$. We then associate each triangle $(v, x, w) \in T_{v}$ with a unique edge $e \in E_{v}$, as defined next.

Definition 1. We say that a triangle $(v, x, w) \in T_{v}$ is associated with the directed edge $\overrightarrow{(v, x)}$ if $x \prec w$, and to $\overrightarrow{(v, w)}$ otherwise. For a directed edge $\vec{e}=\overrightarrow{(v, x)}$ we let $T_{\vec{e}}$ denote the set of triangles that it is associated with, that is, the set of triangles $(v, x, w)$ such that $x \prec w$.

Since it will always be clear from the context from which vertex an edge we consider originates, for the sake of succinctness, we drop the directed notation and use the notation $T_{e}$. We let $t_{e}=\left|T_{e}\right|$, and for a fixed vertex $v$, we get $t_{v}=\sum_{e \in E_{v}} t_{e}$.

In all the follows we assume that $\epsilon<1 / 2$, and otherwise we run the algorithm with $\epsilon=1 / 2$.

\subsection{Heavy and light vertices}

Definition 2. We say that a vertex $v$ is heavy if $d_{v}>\frac{2 \bar{m}}{(\epsilon \bar{t})^{1 / 3}}$ or if $t_{v}>\frac{2 \bar{t}^{2 / 3}}{\epsilon^{1 / 3}}$. If $v$ is such that $d_{v} \leq \frac{2 \bar{m}}{(\epsilon \bar{t})^{1 / 3}}$ and $t_{v} \leq \frac{\bar{t}^{2 / 3}}{2 \epsilon^{1 / 3}}$, then we say that $v$ is light.

We shall say that a partition $(H, L)$ of $V$ is appropriate (with respect to $\bar{m}$ and $\bar{t}$ ) if every heavy vertex belongs to $H$ and every light vertex belongs to $L$.

Note that for an appropriate partition $(H, L)$ both $H$ and $L$ may contain vertices that are neither heavy nor light (but no light vertex belongs to $H$ and no heavy vertex belongs to $L$ ).

For a fixed partition $(H, L)$ we associate with each triangle $\Delta$ a weight depending on the number of its endpoints that belong to $L$. 
Definition 3. For a triangle $\Delta$ we define its weight $w t_{L}(\Delta)$ to be

$$
w t_{L}(\Delta)= \begin{cases}0 & \text { if no endpoints of } \Delta \text { belong to } L \\ 1 / \ell & \text { if } \Delta \text { has } \ell>0 \text { endpoints that belong to } L .\end{cases}
$$

Whenever it is clear for the context, we drop the subscript $L$ and use the notation wt $(\cdot)$ instead of $\mathrm{wt}_{L}(\cdot)$.

Claim 2. If $(H, L)$ is appropriate and Assumption 1 holds, then the number of triangles with weight 0 is at most $c_{H} \cdot \epsilon t$ for some constant $c_{H}$.

Proof. By Assumption 1, the number of vertices $v$ such that $d_{v}$ is greater than $\left(2 \bar{m} /(\epsilon \bar{t})^{1 / 3}\right.$, is at most $2 m /\left(2 \bar{m} /(\epsilon \bar{t})^{1 / 3}\right) \leq 6(\epsilon t)^{1 / 3}$, and the number of vertices $v$ such that $t_{v}>2 \bar{t}^{2 / 3} / \epsilon^{1 / 3}$ is at most $3 t /\left(2 \bar{t}^{2 / 3} / \epsilon^{1 / 3}\right) \leq 6(\epsilon t)^{1 / 3}$. Therefore, there are at most $\left(\begin{array}{c}12(\epsilon t)^{1 / 3} \\ 3\end{array}\right)<2000 \epsilon t$ triangles with all three endpoints in $H$. Setting $c_{H}=2000$ completes the proof.

Definition 4. For any set $T$ of triangles we define $w t(T)=\sum_{\Delta \in T} w t(\Delta)$. For a vertex $v \in L$ we define $w t(v)=\sum_{\Delta \in T_{v}} w t(\Delta)$, and $w t(v)=0$ for $v \in H$.

Lemma 3. For any partition $(H, L), \sum_{v \in L} w t(v) \leq t$. If $(H, L)$ is appropriate and Assumption 1 holds, then $\sum_{v \in L} w t(v) \in\left[t\left(1-c_{H} \cdot \epsilon\right), t\right]$.

Proof. Let $\chi(v, \Delta)$ be an indicator variable such that $\chi(v, \Delta)=1$ if $\Delta$ contains the vertex $v$, and $\chi(v, \Delta)=0$ otherwise. Consider a triangle $\Delta$ that contains $\ell>0$ light vertices. Then

$$
\sum_{v \in L} \chi(v, \Delta)=\ell=1 / \operatorname{wt}(\Delta)
$$

If $\ell=\operatorname{wt}(\Delta)=0$, then the above expression equals 0. By interchanging summations,

$$
\sum_{v \in L} \operatorname{wt}(v)=\sum_{v \in L} \sum_{\Delta \in T_{v}} \operatorname{wt}(\Delta)=\sum_{\Delta \in T} \operatorname{wt}(\Delta) \sum_{v \in L} \chi(v, \Delta)=t-|\{\Delta \mid \operatorname{wt}(\Delta)=0\}| .
$$

Clearly for any partition $(H, L)$ the above expression is at most $t$. On the other hand, If $(H, L)$ is appropriate and Assumption 1 holds, then by Claim 2 we have that $|\{\Delta \mid \operatorname{wt}(\Delta)=0\}| \leq c_{H} \cdot \epsilon t$, and the lemma follows.

Theorem 4. Let $s=\left(c \log (n / \epsilon) / \epsilon^{3}\right) n / \bar{t}^{1 / 3}$ where $c$ is a constant, and let $S$ be a sample of $s$ vertices $v_{1}, v_{2}, \ldots, v_{s}$ that are selected uniformly, independently at random. Then

$$
\mathbf{E}\left[\frac{1}{s} \sum_{i=1}^{s} w t\left(v_{i}\right)\right] \leq \frac{t}{n} .
$$

Furthermore, if $(H, L)$ is appropriate and Assumption 1 holds, then

$$
\mathbf{E}\left[\frac{1}{s} \sum_{i=1}^{s} w t\left(v_{i}\right)\right] \in\left[t\left(1-c_{H} \cdot \epsilon\right) / n, t / n\right]
$$

and for a sufficiently large constant $c$,

$$
\operatorname{Pr}\left[\frac{1}{s} \sum_{i=1}^{s} w t\left(v_{i}\right)<t\left(1-2 c_{H} \cdot \epsilon\right) / n\right]<\epsilon^{2} / n .
$$


Proof. Let $Y$ denote the random variable $Y=\frac{1}{s} \sum_{i=1}^{s} \mathrm{wt}\left(v_{i}\right)$. By the first part of Lemma 3 , $\mathbf{E}\left[\frac{1}{s} \sum_{i=1}^{s} \operatorname{wt}\left(v_{i}\right)\right] \leq t / n$. Now assume that $(H, L)$ is appropriate and Assumption 1 holds. The claim regarding the expected value of $Y$ follows from the second part of Lemma 3, so it remains to prove the claim regarding the deviation from the expected value. Note that $\operatorname{wt}(v) \leq t_{v}$ for every vertex $v$, which for $v \in L$ is at most $\frac{2 \bar{t}^{2 / 3}}{\epsilon^{1 / 3}}$. By the multiplicative Chernoff bound and by Item 1 in Assumption 1,

$$
\operatorname{Pr}[Y<(1-\epsilon) \mathbf{E}[Y]]<\exp \left(-\frac{\epsilon^{2} \mathbf{E}[Y] s}{4 t^{2 / 3} / \epsilon^{1 / 3}}\right)<\exp \left(-\frac{\epsilon^{2} \cdot c \log (n / \epsilon)\left(n / \epsilon \bar{t}^{1 / 3}\right) \cdot t /(2 n)}{4 \bar{t}^{2 / 3} / \epsilon^{1 / 3}}\right)<\frac{\epsilon^{2}}{n},
$$

where the last inequality holds for a sufficiently large constant $c$.

\subsection{A procedure for deciding whether a vertex is heavy}

In this subsection we provide a procedure for deciding (approximately) whether a given vertex $v$ is heavy or light. Recall that a high-level description of the procedure appears in Subsection 1.2.3 of the introduction.

$\operatorname{Heavy}(v)$

1. If $d_{v}>2 \bar{m} /(\epsilon \bar{t})^{1 / 3}$, output heavy.

2. For $i=1,2, \ldots, 10 \log n$ :

(a) For $j=1,2, \ldots, s=20 \bar{m}^{3 / 2} / \epsilon^{2} \bar{t}$ :

i. Select an edge $e \in E_{v}$ uniformly, independently and at random, and let $u$ be the smaller endpoint according to the order $\prec$.

ii. For $k=1,2, \ldots, r=\left\lceil d_{u} / \sqrt{\bar{m}}\right\rceil$ :

A. Pick a neighbor $w$ of $u$ uniformly at random. Let $x$ denote the endpoint of $e$ that is not $v$.

B. If $e$ and $w$ form a triangle and $x \prec w$, set $Z_{k}=d_{u}$, else $Z_{k}=0$.

iii. Set $Y_{j}=\frac{1}{r} \sum_{k} Z_{k}$.

(b) Set $X_{i}=\frac{d_{v}}{s} \sum_{j} Y_{j}$.

3. If the median of the $X_{i}$ variables is greater than $\bar{t}^{2 / 3} / \epsilon^{1 / 3}$, output heavy, else output light.

We have three nested loops, with loop variables $i, j, k$ respectively. We refer to these as "iteration $i$ ", "iteration $j$ ", and "iteration $k$ ".

Lemma 5. For any iteration $i, \operatorname{Pr}\left[\left|X_{i}-t_{v}\right|>\epsilon \cdot \max \left(t_{v}, \bar{t} d_{v} / \bar{m}\right)\right]<1 / 4$.

Proof. Recall that we associate each triangle $(v, x, w) \in T_{v}$ with $(v, x)$ if $x \prec w$ and with $(v, w)$ otherwise, so that we have $t_{v}=\sum_{e \in E_{v}} t_{e}$. For an edge $e=(v, x), t_{e}$ is upper bounded by the number of neighbors $w$ of $x$ such that $x \prec w$. By Claim $1, t_{e} \leq \sqrt{2 m}$.

Fix an iteration $j$ and let $e_{j}$ denote the edge chosen in the $j^{\text {th }}$ iteration and $u_{j}$ denote its smaller degree endpoint. We use $\mathcal{E}_{j}$ to denote the event of $e_{j}$ being chosen. Conditioned on the event $\mathcal{E}_{j}$, the probability that $Z_{k}$ is non-zero is is $t_{e_{j}} / d_{u_{j}}$. Hence,

$$
\mathbf{E}\left[Z_{k} \mid \mathcal{E}_{j}\right]=\frac{t_{e_{j}}}{d_{u_{j}}} \cdot d_{u_{j}}=t_{e_{j}}
$$


and

$$
\operatorname{Var}\left[Z_{k} \mid \mathcal{E}_{j}\right] \leq \mathbf{E}\left[Z_{k}^{2} \mid \mathcal{E}_{j}\right] \leq d_{u_{j}} \cdot \mathbf{E}\left[Z_{k} \mid \mathcal{E}_{j}\right]
$$

By linearity of expectation,

$$
\mathbf{E}\left[Y_{j} \mid \mathcal{E}_{j}\right]=\mathbf{E}\left[\frac{1}{r} \sum_{k=1}^{r} Z_{k} \mid \mathcal{E}_{j}\right]=\frac{1}{r} \sum_{k=1}^{r} \mathbf{E}\left[Z_{k} \mid \mathcal{E}_{j}\right]=t_{e_{j}} .
$$

By the independence of the $Z_{k}$ variables,

$$
\begin{aligned}
\operatorname{Var}\left[Y_{j} \mid \mathcal{E}_{j}\right] & =\operatorname{Var}\left[\frac{1}{r} \sum_{k=1}^{r} Z_{k} \mid \mathcal{E}_{j}\right]=\frac{1}{r^{2}} \sum_{k=1}^{r} \operatorname{Var}\left[Z_{k} \mid \mathcal{E}_{j}\right] \leq \frac{1}{r^{2}} \sum_{k=1}^{r} d_{u_{j}} \cdot \mathbf{E}\left[Z_{k} \mid \mathcal{E}_{j}\right] \\
& =\frac{d_{u_{j}}}{r^{2}} \cdot r \cdot t_{e_{j}} \leq \sqrt{\bar{m}} \cdot t_{e_{j}} .
\end{aligned}
$$

The conditioning can be removed to yield

$$
\mathbf{E}\left[Y_{j}\right]=\sum_{e \in E_{v}} \frac{1}{d_{v}} \cdot \mathbf{E}\left[Y_{j} \mid \mathcal{E}_{j}\right]=\frac{1}{d_{v}} \cdot \sum_{e \in E_{v}} t_{e}=\frac{t_{v}}{d_{v}} .
$$

By the law of total variance, the law of total expectation, the bounds $t_{e_{j}} \leq \sqrt{2 m}$ and $m \leq 6 \bar{m}$, and by Equations (3) and (4):

$$
\begin{aligned}
\operatorname{Var}\left[Y_{j}\right] & =\mathbf{E}_{e_{j}}\left[\operatorname{Var}\left[Y_{j} \mid \mathcal{E}_{j}\right]\right]+\operatorname{Var}_{e_{j}}\left[\mathbf{E}\left[Y_{j} \mid \mathcal{E}_{j}\right]\right] \\
& \leq \mathbf{E}_{e_{j}}\left[\sqrt{\bar{m}} \cdot \mathbf{E}\left[Y_{j} \mid \mathcal{E}_{j}\right]\right]+\operatorname{Var}_{e_{j}}\left[t_{e_{j}}\right] \\
& =\sqrt{\bar{m}} \cdot \mathbf{E}\left[Y_{j}\right]+\mathbf{E}_{e_{j}}\left[t_{e_{j}}^{2}\right] \\
& =\sqrt{\bar{m}} \cdot \mathbf{E}\left[Y_{j}\right]+\frac{1}{d_{v}} \cdot \sum_{e_{j} \in E_{v}} t_{e_{j}}^{2} \\
& \leq \sqrt{\bar{m}} \cdot \mathbf{E}\left[Y_{j}\right]+\sqrt{2 m} \cdot \mathbf{E}\left[Y_{j}\right]<5 \sqrt{\bar{m}} \mathbf{E}\left[Y_{j}\right] .
\end{aligned}
$$

Let $\bar{Y}=\frac{1}{s} \sum_{j} Y_{j}$. By Equation $(5), \mathbf{E}[\bar{Y}]=t_{v} / d_{v}$. By Equation (6),

$$
\begin{aligned}
\operatorname{Var}[\bar{Y}] & =\operatorname{Var}\left[\frac{1}{s} \sum_{j=1}^{s} Y_{j}\right]=\frac{1}{s^{2}} \sum_{j=1}^{s} \operatorname{Var}\left[Y_{j}\right]<\frac{1}{s^{2}} \sum_{j=1}^{s} 5 \sqrt{\bar{m}} \cdot \mathbf{E}\left[Y_{j}\right]=\frac{5 \sqrt{\bar{m}}}{s} \cdot \mathbf{E}\left[\frac{1}{s} \sum_{j=1}^{s} Y_{j}\right] \\
& =\frac{5 \sqrt{\bar{m}}}{s} \mathbf{E}[\bar{Y}]=\frac{5 \sqrt{\bar{m}} \cdot\left(t_{v} / d_{v}\right)}{20 \cdot \bar{m}^{3 / 2} / \epsilon^{2} \bar{t}}=\frac{\epsilon^{2}}{4} \cdot \frac{t_{v}}{d_{v}} \cdot \frac{\bar{t}}{\bar{m}} .
\end{aligned}
$$

By Chebyshev's inequality and Equation (7),

$$
\operatorname{Pr}\left[\left|\bar{Y}-\frac{t_{v}}{d_{v}}\right|>\epsilon \cdot \max \left(\frac{t_{v}}{d_{v}}, \frac{\bar{t}}{\bar{m}}\right)\right] \leq \frac{\operatorname{Var}[\bar{Y}]}{\epsilon^{2} \max \left(t_{v} / d_{v}, \bar{t} / \bar{m}\right)^{2}}<\frac{1}{4} .
$$

Since $X_{i}=d_{v} \cdot \bar{Y}$, we have that $\operatorname{Pr}\left[\left|X_{i}-t_{v}\right|>\epsilon \cdot \max \left(t_{v}, \bar{t} d_{v} / \bar{m}\right)\right]<1 / 4$.

Lemma 6. For every vertex $v$, if $v$ is heavy, then a call to Heavy $(v)$ returns heavy with probability at least $1-1 / n^{2}$. If $v$ is light, then a call to Heavy $(v)$ returns light with probability at least $1-1 / n^{2}$. 
Proof. First consider a heavy vertex $v$. Clearly, if $d_{v}>2 \bar{m} /(\epsilon \bar{t})^{1 / 3}$, then $v$ is declared heavy. Therefore, assume that $t_{v}>2 \bar{t}^{2 / 3} / \epsilon^{1 / 3}$ and $d_{v} \leq 2 \bar{m} /(\epsilon \bar{t})^{1 / 3}$, so that $\bar{t} d_{v} / \bar{m} \leq 2 \bar{t}^{2 / 3} / \epsilon^{1 / 3}$, and hence $\max \left(\bar{t} d_{v} / \bar{m}, t_{v}\right)=t_{v}$. By Lemma 5 , and since $\epsilon<\leq 1 / 2$, for any iteration $i, \operatorname{Pr}\left[\left|X_{i}-t_{v}\right|>\epsilon t_{v}\right]<$ 1/4. Therefore, $\operatorname{Pr}\left[X_{i}<\bar{t}^{2 / 3} / \epsilon^{1 / 3}\right]<1 / 4$, and by Chernoff, the probability that the median of the $X_{i}$ variables (where $i=1, \ldots, 10 \log n$ ) will be greater than $\bar{t}^{2 / 3} / \epsilon^{1 / 3}$ is at least $1-1 / n^{2}$. Hence $\operatorname{Heavy}(v)$ outputs heavy with probability at least $1-1 / n^{2}$.

Now consider a light vertex $v$. Since $d_{v} \leq 2 \bar{m} /(\epsilon \bar{t})^{1 / 3}$ and $t_{v} \leq \bar{t}^{2 / 3} / 2 \epsilon^{1 / 3}$, it holds that $\bar{t} d_{v} / \bar{m} \leq$ $2 \bar{t}^{2 / 3} / \epsilon^{1 / 3}$, implying that $\max \left(\bar{t} d_{v} / \bar{m}, t_{v}\right)=t_{v} \leq 2 \bar{t}^{2 / 3} / \epsilon^{1 / 3}$. Therefore, by Lemma $5, \operatorname{Pr}\left[\left|X_{i}-t_{v}\right|>\right.$ $\left.\epsilon\left(2 \bar{t}^{2 / 3} / \epsilon^{1 / 3}\right)\right]<1 / 4$, and the probability that the median will be less than $\bar{t}^{2 / 3} / \epsilon^{1 / 3}$ is at least $1-1 / n^{2}$. Hence $v$ is declared light with probability at least $1-1 / n^{2}$.

The following is a corollary of Lemma 6 .

Corollary 7. Consider running Heavy for all the vertices in the graph. Let $H$ denote the set of vertices that are declared heavy and let $L$ denote the set of vertices that are declared light. Then, with probability at least $1-1 / n$, the partition $(H, L)$ is appropriate (as defined in Definition 2).

We now turn to analyze the running time of Heavy. The proof will be similar to the complexity analysis of the exact triangle counter of Chiba and Nishizeki [CN85].

Lemma 8. If Item $2 \mathrm{in}$ Assumption 1 holds, then for every vertex $v$ the expected running time of $\operatorname{Heavy}(v)$ is $O^{*}\left(\bar{m}^{3 / 2} / \bar{t}\right)$.

Proof. We first argue that the expected time to generate a single sample of $Y_{j}$ is $O(1)$. Our query model allows for selecting an edge in $E_{v}$ uniformly at random by a single query. If $d_{v} \leq \sqrt{\bar{m}}$, then the degree of the smaller endpoint for any $e \in E_{v}$ is at most $\sqrt{\bar{m}}$. Hence a sample is clearly generated in $O(1)$ time. Suppose that $d_{v}>\sqrt{\bar{m}}$. If an edge $e=(v, u)$ is sampled, then the runtime is $O\left(1+\min \left(d_{v}, d_{u}\right) / \sqrt{\bar{m}}\right)$. Hence, the expected runtime to generate $Y_{j}$ is, up to constant factors, at most:

$$
\frac{1}{d_{v}} \sum_{u \in \Gamma_{v}}\left(1+\frac{\min \left\{d_{v}, d_{u}\right\}}{\sqrt{\bar{m}}}\right) \leq 1+\frac{1}{\sqrt{\bar{m}} \cdot d_{v}} \sum_{u \in \Gamma_{v}} d_{u} \leq 1+\frac{1}{\sqrt{\bar{m}} \cdot d_{v}} \sum_{u \in V} d_{u} \leq 1+\frac{2 m}{\sqrt{\bar{m}} \cdot d_{v}} \leq 5,
$$

where the last inequality follows from Item 2 in Assumption 1

By the above, each iteration of the 'for' loop in Step 2a takes $O(1)$ time in expectation. Therefore, together, all iterations of Step 2a take $O\left(\bar{m}^{3 / 2} /(\epsilon \bar{t})\right)$ time in expectation, and since it is repeated $O(\log n)$ times, the expected running time of the procedure is $\left(\bar{m}^{3 / 2} / \bar{t}\right) \cdot \operatorname{poly}(\log n, 1 / \epsilon)$.

\subsection{Estimating the number of triangles given $\bar{m}$ and $\bar{t}$}

We are now ready to present an algorithm Estimate-with-advice that takes $\bar{m}, \bar{t}$ as input ("advice"), and outputs an estimate of $t$. Later, we employ the the average degree approximation algorithm of Feige [Fei06] and a geometric search to get the bonafide algorithm that estimates $t$ without any initial estimates $\bar{m}$ and $\bar{t}$. Recall that a high-level description of the procedure appears in Subsection 1.2.4 of the introduction. In what follows we rely on the following assumption.

Assumption 2. We will assume that the random coins used by Heavy are fixed in advance, and that the partition $(H, L)$ as defined in Corollary 7 is indeed appropriate.

By Corollary 7 this assumption only adds $1 / n$ to the error probability in all subsequent probability bounds. Recall that we use $c, c_{1}, \ldots$ to denote sufficiently large constants.

Recall that $c_{H}$ is the constant defined in Claim 2. 
Estimate-with-advice $(\bar{m}, \bar{t}, \epsilon)$

1. Sample $s_{1}=c_{1} \epsilon^{-3} \log (n / \epsilon)\left(n / t^{1 / 3}\right)$ vertices, uniformly, independently and at random. Denote the chosen multiset $S$.

2. Set up a data structure to enable sampling vertices in $S$ proportional to their degree. 3. For $i=1,2, \ldots, s_{2}=c_{2} \epsilon^{-4}\left(\log ^{2} n\right)\left(\bar{m}^{3 / 2} / \bar{t}\right)$ :

(a) Sample $v \in S$ proportional to $d_{v}$ and sample $e \in E_{v}$ uniformly at random. Let $u$ be the smaller endpoint according to the order $\prec$. Let $x$ be the endpoint of $e$ that is not $v$.

(b) If $d_{u} \leq \sqrt{\bar{m}}$, set $r=1$ with probability $d_{u} / \sqrt{\bar{m}}$ and set $r=0$ otherwise. If $d_{u}>\sqrt{\bar{m}}$, set $r=\left\lceil d_{u} / \sqrt{\bar{m}}\right\rceil$.

(c) Repeat for $j=1,2, \ldots, r$ :

i. Pick a neighbor $w$ of $u$ uniformly at random.

ii. If $e$ and $w$ do not form a triangle, set $Z_{j}=0$.

iii. If $e$ and $w$ form a triangle and $w \prec x$, set $Z_{j}=0$.

iv. If $e$ and $w$ form a triangle $\Delta$ and $x \prec w$ : call Heavy for all vertices in $\Delta$, and let

$$
Z_{j}=\left\{\begin{array}{ll}
0 & \text { if } \operatorname{Heavy}(v) \text { returned heavy } \\
\max \left(d_{u}, \sqrt{\bar{m}}\right) \cdot \operatorname{wt}(\Delta) & \text { otherwise }
\end{array} .\right.
$$

(d) Set $Y_{i}=\frac{1}{r} \sum_{j=1}^{r} Z_{j}$. (If $r=0$, set $Y_{i}=0$.)

4. Output $X=\frac{n}{s_{1} s_{2}} \cdot\left(\sum_{v \in S} d_{v}\right) \cdot\left(\sum_{i=1}^{s_{2}} Y_{i}\right)$

Theorem 9. For $X$ as defined in Step 4 of Estimate-with-advice, $E[X] \leq t$. Moreover, if $(H, L)$ is appropriate and Assumption 1 holds, then $\mathbf{E}[X] \in\left[t\left(1-c_{H} \cdot \epsilon\right), t\right]$ and $\operatorname{Pr}\left[X<t\left(1-3 c_{H} \cdot \epsilon\right)\right]<$ $3 \epsilon / \log n$.

There are three "levels" of randomness. First is the choice of $S$, second is the choice of $e$ (Step 3a), and finally the $Z_{j}$ 's. When analyzing the randomness in any level, we condition on the previous levels. Before proving the theorem, we present the following definition and claim.

Definition 5. Let $S$ be a multiset of $s_{1}$ vertices. We say that $S$ is good if $\sum_{v \in S} w t(v) / s_{1} \geq t(1-$ $\left.2 c_{H} \cdot \epsilon\right) / n$. We say that $S$ is great if, in addition to being good, $d_{S}=\sum_{v \in S} d_{v} \leq s_{1}(2 m / n)(\log n / \epsilon)$.

Claim 10. Fix the choice of the set $S$, and let $d_{S}=\sum_{v \in S} d_{v}$. For every $i, \mathbf{E}\left[Y_{i} \mid S\right]=d_{S}^{-1} \sum_{v \in S} w t(v)$ and $\operatorname{Var}\left[Y_{i} \mid S\right]<5 \sqrt{\bar{m}} \cdot \mathbf{E}\left[Y_{i} \mid S\right]$.

Proof. This is similar to the argument in Lemma 5 . Let $v_{i}$ be the chosen vertex in the $i^{\text {th }}$ iteration of the algorithm, and let $e_{i}$ be the chosen edge. We refer to this event by $\mathcal{E}_{i}$, and condition over the set $S$ being chosen and the event $\mathcal{E}_{i}$. Denote by $u_{i}$ the lower degree endpoint of $e_{i}$. If Heavy $\left(v_{i}\right)=\operatorname{heavy}$, then $\mathbf{E}\left[Y_{i} \mid S, \mathcal{E}_{i}\right]=0$ and $\operatorname{Var}\left[Y_{i} \mid S, \mathcal{E}_{i}\right]=0$. If Heavy $\left(v_{i}\right)=$ light, then there are two possibilities. If $d_{u_{i}} \leq \sqrt{\bar{m}}$ then,

$$
\mathbf{E}\left[Y_{i} \mid S, \mathcal{E}_{i}\right]=\frac{d_{u_{i}}}{\sqrt{\bar{m}}} \sum_{\Delta \in T_{e_{i}}} \frac{1}{d_{u_{i}}} \cdot \sqrt{\bar{m}} \cdot \operatorname{wt}(\Delta)=\operatorname{wt}\left(T_{e_{i}}\right)
$$


Since the maximum value of $Y_{i}$ in this case is at most $\sqrt{\bar{m}}$,

$$
\operatorname{Var}\left[Y_{i} \mid S, \mathcal{E}_{i}\right] \leq \mathbf{E}\left[Y_{i}^{2} \mid S, \mathcal{E}_{i}\right] \leq \sqrt{\bar{m}} \cdot \mathbf{E}\left[Y_{i} \mid S, \mathcal{E}_{i}\right]
$$

Now consider the case that $d_{u_{i}}>\sqrt{\bar{m}}$. In order to bound the variance of the $Y_{i}$ variables we first analyze the expectation and variance of the $Z_{j}$ variables. Note that $Z_{j}$ is non-zero when a triangle $\Delta \in T_{e_{i}}$ is found. It holds that

$$
\mathbf{E}\left[Z_{j} \mid S, \mathcal{E}_{i}\right]=\sum_{\Delta \in T_{e_{i}}} \frac{1}{d_{u_{i}}} \cdot d_{u_{i}} \cdot \operatorname{wt}(\Delta)=\operatorname{wt}\left(T_{e_{i}}\right),
$$

and

$$
\operatorname{Var}\left[Z_{j} \mid S, \mathcal{E}_{i}\right] \leq d_{u_{i}} \cdot \mathbf{E}\left[Z_{j} \mid S, \mathcal{E}_{i}\right]
$$

By linearity of expectation,

$$
\mathbf{E}\left[Y_{i} \mid S, \mathcal{E}_{i}\right]=\operatorname{wt}\left(T_{e_{i}}\right) .
$$

By independence of the $\left(Z_{j} \mid S, \mathcal{E}_{i}\right)$ variables, linearity of expectation and Equation (9),

$$
\begin{aligned}
\operatorname{Var}\left[Y_{i} \mid S, \mathcal{E}_{i}\right] & =\operatorname{Var}\left[\frac{1}{r} \sum_{j=1}^{r} Z_{j} \mid S, \mathcal{E}_{i}\right]=\frac{1}{r^{2}} \sum_{j=1}^{r} \operatorname{Var}\left[Z_{j} \mid S, \mathcal{E}_{i}\right] \leq \frac{1}{r^{2}} \sum_{j=1}^{r} d_{u_{i}} \cdot \mathbf{E}\left[Z_{j} \mid S, \mathcal{E}_{i}\right] \\
& =\frac{d_{u_{i}}}{r} \cdot \mathbf{E}\left[\frac{1}{r} \sum_{j=1}^{r} Z_{j} \mid S, \mathcal{E}_{i}\right] \leq \sqrt{\bar{m}} \cdot \mathbf{E}\left[Y_{i} \mid S, \mathcal{E}_{i}\right] .
\end{aligned}
$$

We remove the conditioning on $\mathcal{E}_{i}$ :

$$
\mathbf{E}\left[Y_{i} \mid S\right]=\sum_{v \in S \cap L} \frac{d_{v}}{d_{S}} \cdot \frac{1}{d_{v}} \sum_{e \in E_{v}} \mathrm{wt}\left(T_{e}\right)=d_{S}^{-1} \sum_{v \in S \cap L} \sum_{e \in E_{v}} \operatorname{wt}\left(T_{e}\right)=d_{S}^{-1} \sum_{v \in S} \mathrm{wt}(v) .
$$

Recall that by Claim $1, \operatorname{wt}(e) \leq \sqrt{2 m}$. Therefore, by the law of total variance, the law of total expectation, the bound $m \leq 6 \bar{m}$, and Equations (8) and (10),

$$
\begin{aligned}
\operatorname{Var}\left[Y_{i} \mid S\right] & =\mathbf{E}_{e_{i}}\left[\operatorname{Var}\left[Y_{i} \mid S, \mathcal{E}_{i}\right]\right]+\operatorname{Var}_{e_{i}}\left[\mathbf{E}\left[Y_{i} \mid S, \mathcal{E}_{i}\right]\right] \\
& \leq \mathbf{E}_{e_{i}}\left[\sqrt{\bar{m}} \cdot \mathbf{E}\left[Y_{i} \mid S, \mathcal{E}_{i}\right]\right]+\mathbf{E}_{e_{i}}\left[\operatorname{wt}\left(T_{e_{i}}\right)^{2}\right] \\
& \sqrt{\bar{m}} \cdot \mathbf{E}\left[Y_{i} \mid S\right]+\sqrt{2 m} \mathbf{E}_{e_{i}}\left[\operatorname{wt}\left(T_{e_{i}}\right)\right]<5 \sqrt{\bar{m}} \cdot \mathbf{E}\left[Y_{i} \mid S\right]
\end{aligned}
$$

This completes the proof of Claim 10.

Proof of Theorem 9: For a fixed set $S$, let $X_{S}$ denote the sum $X_{S}=\frac{n}{s_{1} s_{2}}\left(\sum_{v \in S} d_{v}\right) \cdot\left(\sum_{i=1}^{s_{2}} Y_{i}\right)$ (as defined in Step 4 of Estimate-with-advice), given that the set $S$ in chosen in Step 1. By the definition of $X_{S}$ and by Claim 10,

$$
\mathbf{E}\left[X_{S}\right]=\frac{n d_{S}}{s_{1}} \mathbf{E}\left[Y_{i} \mid S\right]=\frac{n}{s_{1}} \sum_{v \in S} \mathrm{wt}(v)
$$

By Theorem $4, \mathbf{E}_{S}\left[\frac{1}{s_{1}} \sum_{v \in S} w t(v)\right] \in\left[t\left(1-c_{H} \cdot \epsilon\right) / n, t / n\right]$, implying that

$$
\mathbf{E}\left[X_{S}\right] \in\left[t\left(1-c_{H} \cdot \epsilon\right), t\right]
$$


By Theorem 4, Definition 5 and Assumption 2, $S$ is good with probability at least $1-\epsilon^{2} / n$. The expected value, over $S$, of $d_{S}$ is $\mathbf{E}_{s}\left[d_{S}\right]=s_{1} \cdot \frac{2 m}{n}$. By Markov's inequality,

$$
\operatorname{Pr}_{S}\left[d_{S}>s_{1} \cdot \frac{2 m}{n} \cdot \frac{\log n}{\epsilon}\right]<\frac{\epsilon}{\log n} .
$$

By taking a union bound, the probability that $S$ is great is at least $1-2 \epsilon / \log n$. For a fixed choice of $S$, let $Y_{S}=\frac{1}{s_{2}} \sum_{i=1}^{s_{2}} Y_{i}$. By the independence of the $Y_{i}$ variables and by Claim 10,

$$
\operatorname{Var}\left[Y_{S}\right]=\frac{1}{s_{2}^{2}} \sum_{i=1}^{s_{2}} \operatorname{Var}\left[Y_{i} \mid S\right]<\frac{1}{s_{2}^{2}} \sum_{i=1}^{s_{2}} 5 \sqrt{\bar{m}} \cdot \mathbf{E}\left[Y_{i} \mid S\right]=\frac{5 \sqrt{\bar{m}}}{s_{2}} \cdot \mathbf{E}\left[Y_{S}\right] .
$$

By Chebyshev's inequality, the setting of $s_{2}$ and Equation (12), we get that

$$
\begin{aligned}
\operatorname{Pr}\left[\left|Y_{S}-\mathbf{E}\left[Y_{S}\right]\right|>\epsilon \mathbf{E}\left[Y_{S}\right]\right] & <\frac{\operatorname{Var}\left[Y_{S}\right]}{\epsilon^{2} \cdot \mathbf{E}\left[Y_{S}\right]^{2}} \leq \frac{5 \sqrt{\bar{m}} \cdot \mathbf{E}\left[Y_{S}\right]}{\epsilon^{2}\left(c_{2} \epsilon^{-4} \log ^{2} n\right)\left(\bar{m}^{3 / 2} / \bar{t}\right) \cdot \mathbf{E}\left[Y_{S}\right]^{2}} \\
& =\frac{\epsilon^{2}}{c_{2}\left(\log ^{2} n\right)(\bar{m} / \bar{t}) \cdot \mathbf{E}\left[Y_{S}\right]} .
\end{aligned}
$$

By Claim 10, $\mathbf{E}\left[Y_{S}\right]=d_{S}^{-1} \sum_{v \in S} \mathrm{wt}(v)$, which for a great $S$ is at least

$$
\frac{\left.t\left(1-2 c_{H} \cdot \epsilon\right) / n\right) / s_{1}}{s_{1}(2 m / n)(\epsilon / \log n)} \geq \frac{t}{4 m} \cdot \frac{\epsilon}{\log n}
$$

Therefore, by Assumption 1, for a sufficiently large constant $c_{2}$,

$$
\operatorname{Pr}\left[\left|Y_{S}-\mathbf{E}\left[Y_{S}\right]\right|>\epsilon \mathbf{E}\left[Y_{S}\right]\right] \leq \frac{\epsilon}{\log n} .
$$

By the definition of $X_{S}$ in Step 4 of the algorithm, $X_{S}$ is just a scaling of $Y_{S}$. Therefore,

$$
\operatorname{Pr}\left[\left|X_{S}-\mathbf{E}\left[X_{S}\right]\right|>\epsilon \mathbf{E}\left[X_{S}\right]\right] \leq \frac{\epsilon}{\log n} .
$$

By Equation (11), $\mathbf{E}\left[X_{S}\right]=\frac{n}{s_{1}} \sum_{v \in S} \operatorname{wt}(v)$, which for a great $S$ is at least $t\left(1-2 c_{H} \cdot \epsilon\right)$. Hence, for a great $S$,

$$
\operatorname{Pr}\left[X_{S}<\left(1-3 c_{H} \cdot \epsilon\right) \cdot t\right] \leq \frac{\epsilon}{\log n} .
$$

The probability of $S$ not being great is at most $2 \epsilon / \log n$. We apply the union bound to remove the conditioning, so we get

$$
\operatorname{Pr}\left[X<\left(1-3 c_{H} \cdot \epsilon\right) \cdot t\right] \leq \frac{3 \epsilon}{\log n}
$$

which completes the proof.

Theorem 11. If Item 2 in Assumption 1 holds then the expected running time of Estimate-with-advice is $O^{*}\left(n / \bar{t}^{1 / 3}+\bar{m}^{3 / 2} / \bar{t}\right)$. 
Proof. The sampling of $S$ is done in $O^{*}\left(n / \bar{t}^{1 / 3}\right)$ time. Generating the $Z_{j}$ variables, without the calls to Heavy, takes time $O^{*}\left(\bar{m}^{3 / 2} / \bar{t}\right)$ in expectation, by an argument identical to that in the proof of Lemma 8. Therefore, it remains to bound the running time resulting from calls to Heavy.

Let us compute the expected number of triangles found during the run of the algorithm. In each iteration $i$, conditioned on choosing an edge $e$, the expected number of triangles found is at most $2\left(d_{u} / \sqrt{\bar{m}}\right)\left(t_{e} / d_{u}\right)=2 t_{e} / \sqrt{\bar{m}}$. Averaging over the edges, the expected number of triangles found in a single iteration is at most $6 t /(m \cdot \sqrt{\bar{m}})$, which by Item 2 in Assumption 1 is $O\left(\bar{t} / \bar{m}^{3 / 2}\right)$. There are $O\left(\bar{m}^{3 / 2} / \bar{t}\right) \cdot \operatorname{poly}(\log n, 1 / \epsilon)$ iterations, leading to a total of $O^{*}(1)$ expected triangles. Thus, there are $O^{*}(1)$ expected calls to Heavy, each taking $O^{*}\left(\bar{m}^{3 / 2} / \bar{t}\right)$ time by Lemma 8 . Together with the above, we get an expected running time of $O\left(n / \bar{t}^{1 / 3}+\bar{m}^{3 / 2} / \bar{t}\right) \cdot \operatorname{poly}(\log n, 1 / \epsilon)$.

\subsection{The final algorithm}

We are now ready to present an algorithm that requires no prior knowledge regarding $m$ and $t$.

Estimate $(\epsilon)$

1. Let $\epsilon^{\prime}=\epsilon / 3 c_{H}$, where $c_{H}$ is the constant defined in Claim 2 .

2. Invoke Feige's algorithm [Fei06] for approximating the average degree of a graph $10 \log n$ times. Let $\bar{d}$ be the median value of all invocations.

3. Let $\bar{m}=n \bar{d} / 2$.

4. Let $\widetilde{t}=n^{3}$.

5. While $\widetilde{t} \geq 1$

(a) For $\bar{t}=n^{3}, n^{3} / 2, n^{3} / 4, \ldots, \widetilde{t}$ :

i. For $i=1, \ldots, c \epsilon^{-1} \log \log n$ :

A. Let $X_{i}=$ Estimate-with-advice $\left(\epsilon^{\prime}, \bar{m}, \bar{t}\right)$.

ii. Let $X=\min _{i}\left\{X_{i}\right\}$.

iii. If $X \geq \bar{t}$ return $X$.

(b) Let $\tilde{t}=\tilde{t} / 2$.

Before analyzing the correctness and running time of the algorithm, we present the following simple proposition, whose proof we give for the sake of completeness.

Proposition 12. For every graph $G, t \leq \frac{4}{3} m^{3 / 2}$.

Proof.

$$
t=\frac{1}{3} \sum_{v \in V} \frac{1}{2} t_{v} \leq \frac{1}{6}\left(\sum_{v: d_{v}>\sqrt{m}} t_{v}+\sum_{v: d_{v} \leq \sqrt{m}} 2 d_{v}^{2}\right) \leq \frac{1}{6}\left(2 \sqrt{m} \cdot 2 m+2 \sqrt{m} \sum_{v: d_{v} \leq \sqrt{m}} d_{v}\right) \leq \frac{4}{3} m^{3 / 2} .
$$

Theorem 13. Algorithm Estimate $(\epsilon)$ returns a value $X$, such that $(1-\epsilon) t \leq X \leq$ $(1+\epsilon) t$, with probability at least 5/6. The expected query complexity of the algorithm is $O^{*}\left(n / t^{1 / 3}+\max \left\{m, m^{3 / 2} / t\right\}\right)$ and the expected running time of the algorithm is $O^{*}\left(n / t^{1 / 3}+\right.$ $\left.m^{3 / 2} / t\right)$.

Proof. We first prove that the value of $X$ is as stated in the theorem. Let $d_{\text {avg }}$ denote the average degree of vertices in $G$. The algorithm from [Fei06] returns a value $\bar{d}$ such that, with probability at least $2 / 3, \bar{d} \in\left[d_{\text {avg }} /(2+\gamma), d_{\text {avg }}\right]$ for a constant $\gamma$. Since we take the median value of $10 \log n$ 
invocations, it follows from Chernoff's inequality that $\bar{m}$ is as stated in Item 2 of Assumption 1 with probability at least $1-1 / \operatorname{poly}(n)$. Assume that this is indeed the case.

Before analyzing the algorithm Estimate as described above, first consider executing Step 5a with $\widetilde{t}=1$. That is, rather than running both an outer loop over decreasing values of $\widetilde{t}$ and an inner loop over decreasing values of $\bar{t}$, we only run a single loop over decreasing value of $\bar{t}$, starting with $\bar{t}=n^{3}$. By the first part of Theorem 9 and by Markov's inequality, for each value of $\bar{t}$ and for each $i, \operatorname{Pr}\left[X_{i} \leq(1+\epsilon) t\right]>\epsilon / 2$, where $X_{i}$ as defined in Step 5(a)iA. Therefore, for each value of $\bar{t}$, the minimum estimate $X$ (as defined in Step 5(a)iii) is at most $(1+\epsilon) t$, with probability at least $1-1 / \log ^{3} n$. It follows that for each $\bar{t}$ such that $\bar{t}>2 t$, we have that $X<\bar{t}$ with probability at least $1-1 / \log ^{3} n$, and the algorithm will continue with $\bar{t}=\bar{t} / 2$. Once we reach a value of $\bar{t}$ for which $t / 4 \leq \bar{t} \leq t / 2$, Item 1 in Assumption 1, regarding $\bar{t}$, holds. By the second part of Theorem 9 , $X_{i} \in[(1-\epsilon) t,(1+\epsilon) t]$ for every $i$ with probability at least $1-c / \log n$. Hence, we have that

$$
\bar{t} \leq \frac{1}{2} t \leq(1-\epsilon) t \leq X \leq(1+\epsilon) t
$$

with probability at least $1-c / \log n$. Therefore, we halt and return correct $X$.

If however we do reach a value $\bar{t}$ such that $\bar{t} \leq t / 4$, since Assumption 1 does not hold, we cannot lower bound $X$, implying that we can no longer bound the probability that $X<\bar{t}$. Therefore we might continue running with decreasing values of $t$, causing the running time to exceed the desired bound of $O^{*}\left(n / t^{1 / 3}+m^{3 / 2} / t\right)$. In order to avoid this scenario, we run both an outer loop over $\widetilde{t}$ and an inner loop over $\bar{t}$. Specifically, starting with $\widetilde{t}=n^{3}$, whenever we halve $\tilde{t}$, we run over all values of $\bar{t}=n^{3}, n^{3} / 2, \ldots$, until we reach $\widetilde{t}$. This implies that for every value of $\widetilde{t}>2 t$ the probability of returning an incorrect estimate, that is, outside the range of $(1-\epsilon) t \leq X \leq(1+\epsilon) t$, is at most $1-1 / \log ^{2} n$. On the other hand, for values of $\widetilde{t}$ such that $\widetilde{t} \leq t / 2$ the probability of returning a correct estimate (within $(1-\epsilon) t \leq X \leq(1+\epsilon) t$ ) is at least $1-c / \log n$. A union bound over all failure probabilities gives a success probability of at least $5 / 6$.

We now turn to analyze the query complexity and running time of the algorithm. By [Fei06], the expected running time of the average degree approximation algorithm is $O^{*}(n / \sqrt{m})$. By Theorem 11, conditioned on $\bar{m}$ satisfying Item 2 in Assumption 1, the expected running time of Estimate-with-advice $(\epsilon, \bar{m}, \bar{t})$ is $O^{*}\left(n / t^{1 / 3}+\bar{m}^{3 / 2} / t\right)$. It follows from Proposition 12 that $n / \sqrt{m}=O\left(n / t^{1 / 3}\right)$, implying that the running time is determined by the value of $\bar{m}$ and by the smallest value of $\bar{t}$ that Estimate-with-advice $(\epsilon, \bar{m}, \bar{t})$ is invoked with.

Recall that whenever we halve the value of $\tilde{t}$, we run with all values $\bar{t}=n^{3}, n^{3} / 2, \ldots$. This, together with the fact that when running with $t / 4 \leq \bar{t} \leq t / 2$ we halt with probability at least $1-c / \log n$, implies that the probability of reaching a value $\tilde{t}=t / 2^{k}$ is at most $(c / \log n)^{k}$. Therefore, the expected running time, conditioned on $\bar{m}$ satisfying Item 2 in Assumption 1 , is bounded by

$$
\log ^{2} n \cdot O^{*}\left(\frac{n}{t^{1 / 3}}+\frac{\bar{m}^{3 / 2}}{t}\right)+\sum_{k=1}^{\log n}(c / \log n)^{k} \cdot 2^{k} \cdot O^{*}\left(\frac{n}{t^{1 / 3}}+\frac{\bar{m}^{3 / 2}}{t}\right)=O^{*}\left(\frac{n}{t^{1 / 3}}+\frac{\bar{m}^{3 / 2}}{t}\right)
$$

Now consider the value of $\bar{m}$ computed in Step 3 of Estimate $(\epsilon)$. As stated previously, with probability at least $1-1 / \operatorname{poly}(n)$ (e.g., $\left.1-1 / n^{4}\right)$, the estimate $\bar{m}$ is within a constant factor from $m$. Therefore the expected running time of the algorithm (without the conditioning on the value of $\bar{m}$ ) is bounded by

$$
\left(1-\frac{1}{n^{4}}\right) \cdot O^{*}\left(\frac{n}{t^{1 / 3}}+\frac{m^{3 / 2}}{t}\right)+\frac{1}{n^{4}} \cdot O\left(n^{3}\right)=O^{*}\left(\frac{n}{t^{1 / 3}}+\frac{m^{3 / 2}}{t}\right) .
$$


Observe that we can always assume that the algorithm does not perform queries it can answer by itself. That is, we can allow the algorithm to save all the information it obtained from past queries, and assume it does not query for information it can deduce from its past queries. Further observe that any pair query is preceded by a neighbor query. Therefore, if at any point the algorithm performs more than $2 \bar{m}$ queries, it can abort. It follows that the expected query complexity is $O^{*}\left(n / t^{1 / 3}+\min \left\{m, m^{3 / 2} / t\right\}\right)$.

\section{A Lower Bound}

In this section we present a lower bound on the number of queries necessary for estimating the number of triangles in a graph. Since we sometimes refer to the number of triangles in different graphs, we use the notation $t(G)$ for the number of triangles in a graph $G$. Our lower bound matches our upper bound in terms of the dependence on $n, m$ and $t(G)$, up to polylogarithmic factors in $n$ and the dependence in $1 / \epsilon$. In what follows, when we refer to approximation algorithms for the number of triangles in a graph, we mean multiplicative-approximation algorithms that output with high constant probability an estimation $\widehat{t}$ such that $t(G) / C \leq \widehat{t} \leq C \cdot t(G)$ for some predetermined approximation factor $C$.

We consider multiplicative-approximation algorithms that are allowed the following three types of queries: Degree queries, pair queries and random new-neighbor queries. Degree queries and pair queries are as defined in Section 2. A random new-neighbor query $q_{i}$ is a single vertex $u$ and the corresponding answer is a vertex $v$ such that $(u, v) \in E$ and the edge $(u, v)$ is selected uniformly at random among the edges incident to $u$ that have not yet been observed by the algorithm. In Corollary 34 we show that this implies a lower bound when the algorithm may perform (standard) neighbor queries instead of random new-neighbor queries.

We first give a simple lower bound that depends on $n$ and $t(G)$.

Theorem 14. Any multiplicative-approximation algorithm for the number of triangles in a graph must perform $\Omega\left(\frac{n}{t(G)^{1 / 3}}\right)$ queries, where the allowed queries are degree queries, pair queries and random new-neighbor queries.

Proof. For every $n$ and every $1 \leq t \leq\left(\begin{array}{l}n \\ 3\end{array}\right)$ we next define a graph $G_{1}$ and a family of graphs $\mathcal{G}_{2}$ for which the following holds. The graph $G_{1}$ is the empty graph over $n$ vertices. In $\mathcal{G}_{2}$, each graph consists of a clique of size $\left\lfloor t^{1 / 3}\right\rfloor$ and an independent set of size $n-\left\lfloor t^{1 / 3}\right\rfloor$. See Figure 1 for an illustration. Within $\mathcal{G}_{2}$ the graphs differ only in the labeling of the vertices. By construction, $G_{1}$ contains no triangles and each graph in $\mathcal{G}_{2}$ contains $\Theta(t)$ triangles. Clearly, unless the algorithm "hits" a vertex in the clique it cannot distinguish between the two cases. The probability of hitting such a vertex in a graph selected uniformly at random from $\mathcal{G}_{2}$ is $\left\lfloor t^{1 / 3}\right\rfloor / n$. Thus, in order for this event to occur with high constant probability, $\Omega\left(\frac{n}{t^{1 / 3}}\right)$ queries are necessary.

We next state our main theorem.

Theorem 15. Any multiplicative-approximation algorithm for the number of triangles in a graph must perform at least $\Omega\left(\min \left\{\frac{m^{3 / 2}}{t(G)}, m\right\}\right)$ queries, where the allowed queries are degree queries, pair queries and random new-neighbor queries.

For every $n$, every $1 \leq m \leq\left(\begin{array}{l}n \\ 2\end{array}\right)$ and every $1 \leq t \leq \min \left\{\left(\begin{array}{l}n \\ 3\end{array}\right), m^{3 / 2}\right\}$ we define a graph $G_{1}$ and a family of graphs $\mathcal{G}_{2}$ for which the following holds. The graph $G_{1}$ and all the graphs in $\mathcal{G}_{2}$ have $n$ vertices and $m$ edges. For the graph $G_{1}, t\left(G_{1}\right)=0$, and for every graph $G \in \mathcal{G}_{2}, t(G)=\Theta(t)$. 

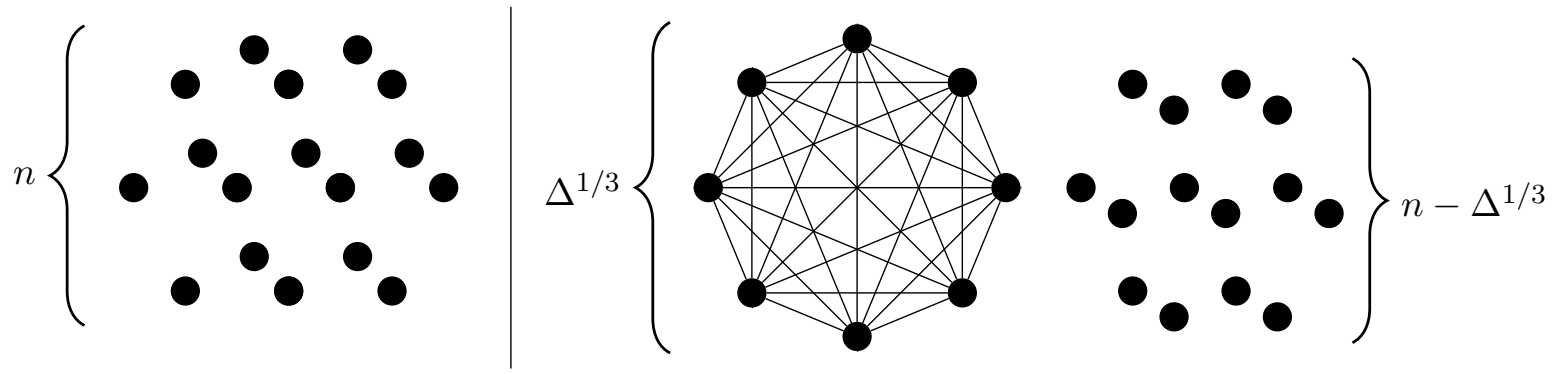

Figure 1: An illustration of the two families.

We prove it is necessary to perform $\Omega\left(\min \left\{\frac{m^{3 / 2}}{t}, m\right\}\right)$ queries in order to distinguish with high constant probability between $G_{1}$ and a random graph in $\mathcal{G}_{2}$. For the sake of simplicity, in everything that follows we assume that $\sqrt{m}$ is even.

We prove that for values of $t$ such that $t<\frac{1}{4} \sqrt{m}$, at least $\Omega(m)$ queries are required, and for values of $t$ such that $t \geq \sqrt{m}$ at least $\Omega\left(\frac{m^{3 / 2}}{t}\right)$ queries are required.

We delay the discussion on the former case to Subsection 4.4, and start with the case that $t \geq \sqrt{m}$. Our construction of $\mathcal{G}_{2}$ depends on the value of $t$ as a function of $m$ where we deal separately with the following two ranges of $t$ :

1. $t \in\left[\Omega(m), O\left(m^{3 / 2}\right)\right]$.

2. $t \in[\Omega(\sqrt{m}, O(m)]$.

We prove that for every $t$ as above, $\Omega\left(m^{3 / 2} / t\right)$ queries are needed in order to distinguish between the graph $G_{1}$ and a random graph in $\mathcal{G}_{2}$. Observe that by Proposition 12, for every graph $G$, it holds that $t(G)=O\left(\mathrm{~m}^{3 / 2}\right)$. Hence, the above ranges indeed cover all the possible values of $t$ as a function of $m$.

A high level discussion of the lower bound. The constructions for the different ranges of $t \geq \sqrt{m}$ are all based on the same basic idea, and have the following in common. In all construction for $t$ as above, $G_{1}$ consists of a complete bipartite graph $(L \cup R, E)$ with $|L|=|R|=\sqrt{m}$ and an independent set of $n-2 \sqrt{m}$ vertices. The basic structure of the graphs in the family $\mathcal{G}_{2}$ is the same as that of $G_{1}$ with the following modifications:

- For every value of $t$, we add $t / \sqrt{m}$ edges between vertices in $L$ (and similarly in $R$ ). Since each edge contributes (roughly) $\sqrt{m}$ triangles, this gives the desired total number of triangles in the graph. In the case that $t=m$ this is done by adding a perfect matching within $L$ and a perfect matching within $R$. In the case that $t>m$ we add several such perfect matchings, and in the case that $\sqrt{m} \leq t \leq m / 4$ we add a (non-perfect) matching of size $t / \sqrt{m}$.

- In order to maintain the degrees of all the vertices in the bipartite component, we remove edges between vertices in $L$ and $R$.

For an illustration of the case $t=m$, see Figure 2. In what follows we assume that the algorithm knows in advance which vertices are in $L$ and which are in $R$, and consider only the bipartite component of the graphs. In order to give the intuition for the $m^{3 / 2} / t$ lower bound we consider each type of query separately, starting with degree queries. 
Since both in the graph $G_{1}$ and in all the graphs in $\mathcal{G}_{2}$, all the vertices in $L \cup R$ have the same degree (of $\sqrt{m}$ ), degree queries do not reveal any information that is useful for distinguishing between the two.

As for pair queries, unless the algorithm queries a pair in $L \times L$ (or $R \times R$ ) and receives a positive answer, or queries a pair in $L \times R$ and receives a negative answer, the algorithm cannot distinguish between the bipartite component of the graph $G_{1}$ and those of the graphs in $\mathcal{G}_{2}$. We refer to these pairs as witness pairs. Roughly speaking, since there are $\Theta(t / \sqrt{m})$ such pairs, and $m$ pairs in total, it takes $\Omega\left(m^{3 / 2} / t\right)$ queries in order to "catch a witness pair".

We are left to deal with neighbor queries. Here too, distinguishing between the graph $G_{1}$ and the graphs in $\mathcal{G}_{2}$ can be done by "catching a witness". That is, if the algorithm queries for a neighbor of a vertex in $L$ and the answer is another vertex in $L$ (analogously for a vertex in $R$ ). As before, the probability for hitting such a witness pair is small. However, there is another source of difference resulting from neighbor queries. When the algorithm queries a vertex $v \in L$ there is a difference in the conditional distribution on answers $v \in R$ when the answer is according to the graph $G_{1}$ or according to a graph in the family $\mathcal{G}_{2}$. The reason for the difference, is that in the graph $G_{1}$ every vertex has exactly $\sqrt{m}$ neighbors in the opposite side, while for graphs in $\mathcal{G}_{2}$, each vertex has $\Theta(\sqrt{m}-t / m)$ neighbors in the opposite side (for the range $\Omega(\sqrt{m}) \leq t \leq O(m)$ this is true on average). We prove that this difference in sufficiently small so as to ensure the $\Omega\left(m^{3 / 2} / t\right)$ lower bound.

Our formal analysis is based on defining two processes that interact with an algorithm for approximating the number of triangles, denoted ALG. The first process answer queries according to $G_{1}$, and the second process answers queries while constructing a uniformly selected graph in $\mathcal{G}_{2}$. An interaction between ALG and each of these processes induces a distribution over sequences of queries and answers. We prove that if the number of queries performed by ALG is smaller than $m^{3 / 2} /(c t)$ for a sufficiently large constant $c$, then the statistical distance between the two distributions is a small constant.

We start by addressing the case that $t=m$ in Subsection 4.1 , and deal with the case that $m<t \leq \frac{m^{3 / 2}}{8}$ in Subsection 4.2, and with the case that $\sqrt{m} \leq t \leq \frac{m}{4}$ in Subsection 4.3.

Before embarking on the proof for $t=m$, we introduce the notion of a knowledge graph (as defined previously in e.g., [GR02]), which will be used in all lower bound proofs. Let ALG be an algorithm for approximating he number of triangles, which performs $Q$ queries. Let $q_{t}$ denote its $t^{\text {th }}$ query and let $a_{t}$ denote the corresponding answer. Then ALG is a (possibly probabilistic) mapping from query-answer histories $\pi \triangleq\left\langle\left(q_{1}, a_{1}\right), \ldots,\left(q_{t}, a_{t}\right)\right\rangle$ to $q_{t+1}$, for every $t<Q$, and to $\mathbb{N}$ for $t=Q$.

We assume that the mapping determined by the algorithm is determined only on histories that are consistent with the graph $G_{1}$ or one of the graphs in $\mathcal{G}_{2}$. Any query-answer history $\pi$ of length $t$ can be used to define a knowledge graph $G_{\pi}^{k n}$ at time $t$. Namely, the vertex set of $G_{\pi}^{k n}$ consists of $n$ vertices. For every new-neighbor query $u_{i}$ answered by $v_{i}$ for $i \leq t$, the knowledge graph contains the edge $\left(u_{i}, v_{i}\right)$, and similarly for every pair query $\left(u_{j}, v_{j}\right)$ that was answered by 1 . In addition, for every pair query $\left(u_{i}, v_{i}\right)$ that is answered by 0 , the knowledge graph maintains the information that $\left(u_{i}, v_{i}\right)$ is a non-edge. The above definition of the knowledge graph is a slight abuse of the notation of a graph since $G_{\pi}^{k n}$ is a subgraph of the graph tested by the algorithm, but it also contains additional information regarding queried pairs that are not edges. For a vertex $u$, we denote its set of neighbors in the knowledge graph by $\Gamma_{\pi}^{k n}(u)$, and let $d_{\pi}^{k n}(u)=\left|\Gamma_{\pi}^{k n}(u)\right|$. We denote by $N_{\pi}^{k n}(u)$ the set of vertices $v$ such that $(u, v)$ is either an edge or a non-edge in $G_{\pi}^{k n}$. 


\subsection{A lower bound for $t=m$}

\subsubsection{The lower-bound construction}

The graph $G_{1}$ has two components. The first component is a complete bipartite graph with $\sqrt{m}$ vertices on each side, i.e, $K_{\sqrt{m}, \sqrt{m}}$, and the second component is an independent set of size $n-2 \sqrt{m}$. We denote by $L$ the set of vertices $\ell_{1}, \ldots, \ell_{\sqrt{m}}$ on the left-hand side of the bipartite component and by $R$ the set of vertices $r_{1}, \ldots, r_{\sqrt{m}}$ on its right-hand side. The graphs in the family $\mathcal{G}_{2}$ have the same basic structure with a few modifications. We first choose for each graph a perfect matching $M^{C}$ between the two sides $R$ and $L$ and remove the edges in $M^{C}$ from the graph. We refer to the removed matching as the "red matching" and its pairs as "crossing non-edges" or "red pairs". Now, we add two perfect matching from $L$ to $L$ and from $R$ to $R$, denoted $M^{L}$ and $M^{R}$ respectively. We refer to these matchings as the blue matchings and their edges as "non-crossing edges" or "blue pairs". Thus for each choice of three perfect matchings $M^{C}, M^{L}$ and $M^{R}$ as defined above, we have a corresponding graph in $\mathcal{G}_{2}$.

Consider a graph $G \in \mathcal{G}_{2}$. Clearly, every blue edge participate in $\sqrt{m}-2$ triangles. Since, every triangle in the graph contains exactly one blue edge, there are $2 \sqrt{m} \cdot(\sqrt{m}-2)=\Theta(m)$ triangles in $G$.
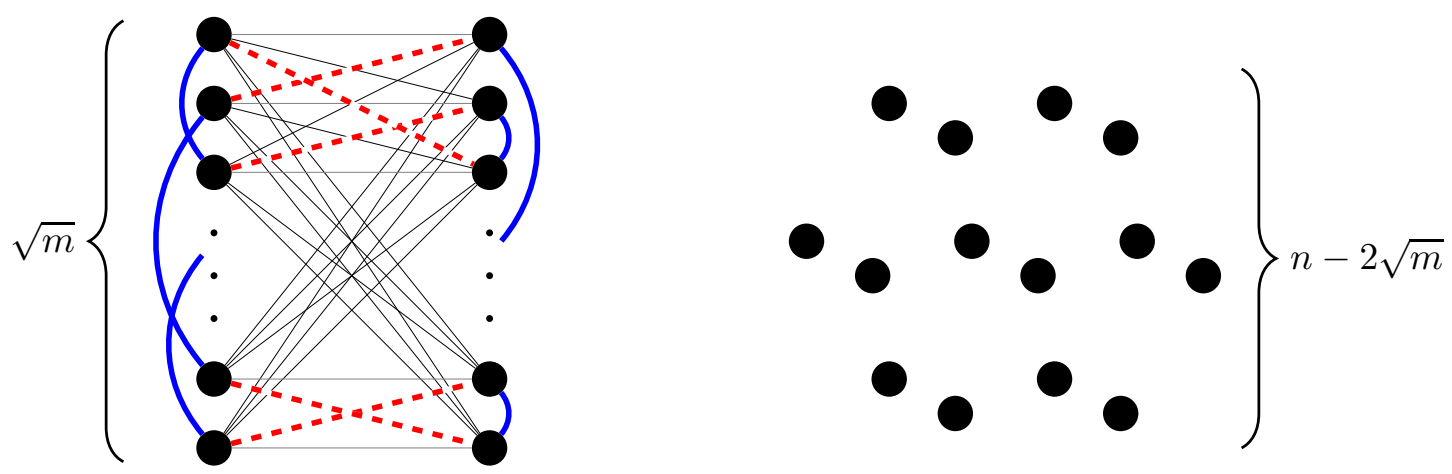

Figure 2: An illustration of the family $\mathcal{G}_{2}$ for $t=m$.

\subsubsection{Definition of the processes $P_{1}$ and $P_{2}$}

In what follows we describe two random processes, $P_{1}$ and $P_{2}$, which interact with an arbitrary algorithm ALG. The process $P_{1}$ answers ALG's queries consistently with $G_{1}$. The process $P_{2}$ answers ALG's queries while constructing a uniformly selected random graph from $\mathcal{G}_{2}$. We assume without loss of generality that ALG does not ask queries whose answers can be derived from its knowledge graph, since such queries give it no new information. For example, ALG does not ask a pair query about a pair of vertices that are already known to be connected by an edge due to a neighbor query. Also, we assume ALG knows in advance which vertices belong to $L$ and which to to $R$, so that ALG need not query vertices in the independent set. Since the graphs in $\mathcal{G}_{2}$ differ from $G_{1}$ only in the edges of the subgraph induced by $L \cup R$, we think of $G_{1}$ and graphs in $\mathcal{G}_{2}$ as consisting only of this subgraph. Finally, since in our constructions all the vertices in $L \cup R$ have the same degree of $\sqrt{m}$, we assume that no degree queries are performed.

For every, $Q$, every $t \leq Q$ and every query-answer history $\pi$ of length $t-1$ the process $P_{1}$ answers the $t^{\text {th }}$ query of the algorithm consistently with $G_{1}$. Namely:

- For a pair query $q_{t}=(u, v)$ if the pair $(u, v)$ is a crossing pair in $G_{1}$, then the process replies 1 , and otherwise it replies 0 . 
- For a random new-neighbor query $q_{t}=u$ the process answers with a random neighbor of $u$ that has yet been observed by the algorithm. That is, for every vertex $v$ such that $v \in \Gamma(u) \backslash \Gamma_{\pi}^{k n}(u)$ the process replies $a_{t}=v$ with probability $1 /\left(\sqrt{m}-d_{\pi}^{k n}(u)\right)$.

The process $P_{2}$ is defined as follows:

- For a query-answer history $\pi$ we denote by $\mathcal{G}_{2}(\pi) \subset \mathcal{G}_{2}$ the subset of graphs in $\mathcal{G}_{2}$ that are consistent with $\pi$.

- For every $t \leq Q$ and every query-answer history $\pi$ of length $t-1$, the process $P_{2}$ selects a graph in $\mathcal{G}_{2}$ uniformly at random and answers the $t^{\text {th }}$ query as follows.

1. If the $t^{\text {th }}$ query is a pair query $q_{t}=(u, v)$, then $P_{2}$ answers the query $q_{t}$ according to the selected graph.

2. If the $t^{\text {th }}$ query is a random new-neighbor query $q_{t}=u_{t}$, then $P_{2}$ 's answer is a uniform new neighbor of $u_{t}$ in the selected graph.

- After all queries are answered (i.e., after $Q$ queries), uniformly choose a random graph $G$ from $\mathcal{G}_{2}(\pi)$.

For a query-answer history $\pi$ of length $Q$ we denote by $\pi^{\leq t}$ the length $t$ prefix of $\pi$ and by $\pi^{\geq t}$ the $Q-t+1$ suffix of $\pi$.

We note that the selected graph is only used to answer the $t^{\text {th }}$ query and is then "discarded back to" the remaining graphs that are consistent with that answer (and all previous answers in $\pi)$.

Claim 16. Let $\pi$ be a query-answer history of length $t-1$. We use $\circ$ to denote concatenation.

- If the $t^{\text {th }}$ query is a pair query, then $a_{t}=1$ with probability

$$
\frac{\left|\mathcal{G}_{2}\left(\pi \circ\left(q_{t}, 1\right)\right)\right|}{\left|\mathcal{G}_{2}(\pi)\right|}
$$

and $a_{t}=0$ with probability

$$
\frac{\left|\mathcal{G}_{2}\left(\pi \circ\left(q_{t}, 0\right)\right)\right|}{\left|\mathcal{G}_{2}(\pi)\right|} .
$$

- If the $t^{\text {th }}$ query is a random new-neighbor query $q_{t}=u_{t}$, then for every $v \in V \backslash \Gamma_{\pi}^{k n}(u)$ the probability that the process $P_{2}$ answers $a_{t}=v$ is

$$
\frac{\left|\mathcal{G}_{2}\left(\pi \circ\left(q_{t}, v\right)\right)\right|}{\left|\mathcal{G}_{2}(\pi)\right|} \cdot \frac{1}{\sqrt{m}-d_{\pi}^{k n}\left(u_{t}\right)} .
$$

If $v \in \Gamma_{\pi}^{k n}(u)$ then the probability that $P_{2}$ answers $a_{t}=v$ is 0 .

Proof. First consider a pair query $q_{t}=\left(u_{t}, v_{t}\right)$. The probability that $\left(u_{t}, v_{t}\right)$ is an edge in the graph chosen by the process $P_{2}$ is the fraction of graphs in $\mathcal{G}_{2}(\pi)$ in which $\left(u_{t}, v_{t}\right)$ is an edge. This is exactly $\frac{\left|\mathcal{G}_{2}\left(\pi \circ\left(q_{t}, 1\right)\right)\right|}{\left|\mathcal{G}_{2}(\pi)\right|}$. Similarly, the probability of choosing a graph in which $\left(u_{t}, v_{t}\right)$ is not an edge is $\frac{\left|\mathcal{G}_{2}\left(\pi \circ\left(q_{t}, 0\right)\right)\right|}{\left|\mathcal{G}_{2}(\pi)\right|}$.

Now consider a random new-neighbor query $q_{t}=u_{t}$. We start with the case that $v \in V \backslash \Gamma_{\pi}^{k n}$. The probability that $v$ is chosen by $P_{2}$ is the probability that a graph $G$ in which $v$ is a neighbor of $u_{t}$ is chosen in the first step, and that $v$ is the chosen new neighbor among all of $u$ 's neighbors 
in the second step. Since there are $\left|\mathcal{G}_{2}(\pi \circ(u, v))\right|$ graphs in which $v$ is a neighbor of $u_{t}$, and $u_{t}$ has $\sqrt{m}-d_{\pi}^{k n}\left(u_{t}\right)$ neighbors, this happens with probability

$$
\frac{\left|\mathcal{G}_{2}(\pi \circ(u, v))\right|}{\left|\mathcal{G}_{2}\right|} \cdot \frac{1}{\sqrt{m}-d_{\pi}^{k n}\left(u_{t}\right)} .
$$

For a vertex $v$ such that $v \notin V \backslash \Gamma_{\pi}^{k n}$, in every graph $G \in \mathcal{G}_{2}, v$ is not a neighbor of $u_{t}$, implying that the probability that the process replies $a_{t}=v$ is 0 .

Lemma 17. For every algorithm $A L G$, the process $P_{2}$, when interacting with $A L G$, answers $A L G$ 's queries according to a uniformly generated graph $G$ in $\mathcal{G}_{2}$.

Proof. Consider a specific graph $G \in \mathcal{G}_{2}$. Let $\pi$ be the query-answer history generated by the interaction between ALG and $P_{2}$. Let $Q$ be the number of queries performed during the interaction. The probability that $G$ is the resulting graph from that interaction is

$$
\begin{gathered}
\operatorname{Pr}\left[G \in \mathcal{G}_{2}\left(\pi^{\leq 1}\right)\right] \cdot \operatorname{Pr}\left[G \in \mathcal{G}_{2}\left(\pi^{\leq 2}\right) \mid G \in \mathcal{G}_{2}\left(\pi^{\leq 1}\right)\right] \cdot \ldots \cdot \operatorname{Pr}\left[G \in \mathcal{G}_{2}\left(\pi^{\leq Q}\right) \mid G \in \mathcal{G}_{2}\left(\pi^{\leq Q-1}\right)\right] \cdot \frac{1}{\left|\mathcal{G}\left(\pi^{\leq Q}\right)\right|} \\
=\frac{\left|\mathcal{G}_{2}\left(\pi^{\leq 1}\right)\right|}{\left|\mathcal{G}_{2}\right|} \cdot \frac{\left|\mathcal{G}_{2}\left(\pi^{\leq 2}\right)\right|}{\left|\mathcal{G}_{2}\left(\pi^{\leq 1}\right)\right|} \cdot \ldots \cdot \frac{\left|\mathcal{G}_{2}\left(\pi^{\leq Q}\right)\right|}{\left|\mathcal{G}_{2}\left(\pi^{\leq Q-1}\right)\right|} \cdot \frac{1}{\left|\mathcal{G}_{2}\left(\pi^{Q}\right)\right|}=\frac{1}{\left|\mathcal{G}_{2}\right|},
\end{gathered}
$$

and the lemma follows.

For a fixed algorithm ALG that performs $Q$ queries, and for $b \in\{1,2\}$, let $\mathcal{D}_{\text {ALG }}^{b}$ denote the distribution on query-answers histories of length $Q$ induced by the interaction between ALG and $P_{b}$. We shall show that for every algorithm ALG that performs at most $Q=\frac{m^{3 / 2}}{100 t}$ queries, the statistical distance between $\mathcal{D}_{1}^{\mathrm{ALG}}$ and $\mathcal{D}_{2}^{\mathrm{ALG}}$, denoted $d\left(\mathcal{D}_{1}^{\mathrm{ALG}}, \mathcal{D}_{2}^{\mathrm{ALG}}\right)$, is at most $\frac{1}{3}$. This will imply that the lower bound stated in Theorem 15 holds for the case that $t(G)=m$. In order to obtain this bound we introduce the notion of a query-answer witness pair, defined next.

Definition 6. We say that $A L G$ has detected a query-answer witness pair in three cases:

1. If $q_{t}$ is a pair query for a crossing pair $\left(u_{t}, v_{t}\right) \in L \times R$ and $a_{t}=0$.

2. If $q_{t}$ is a pair query for a non-crossing pair $\left(u_{t}, v_{t}\right) \in(L \times L) \cup(R \times R)$ and $a_{t}=1$.

3. If $q_{t}=u_{t}$ is a random new-neighbor query and $a_{t}=v$ for some $v$ such that $\left(u_{t}, v\right)$ is a non-crossing pair.

We note that the source of the difference between $\mathcal{D}_{1}^{\mathrm{ALG}}$ and $\mathcal{D}_{2}^{\mathrm{ALG}}$ is not only due to the probability that the query-answer history contains a witness pair (which is 0 under $\mathcal{D}_{1}^{\text {ALG }}$ and non-0 under $\mathcal{D}_{2}^{\mathrm{ALG}}$ ). There is also a difference in the distribution over answers to random new neighbor queries when the answers do not result in witness pairs (in particular when we condition on the query-answer history prior to the $t^{\text {th }}$ query). However, the analysis of witness pairs serves us also in bounding the contribution to the distance due to random new neighbor queries that do not result in a witness pairs.

Let $w$ be a "witness function", such that for a pair query $q_{t}$ on a crossing pair, $w\left(q_{t}\right)=0$, and for a non-crossing pair, $w\left(q_{t}\right)=1$. The probability that ALG detects a witness pair when $q_{t}$ is a pair query $\left(u_{t}, v_{t}\right)$ and $\pi$ is a query-answer history of length $t-1$, is

$$
\operatorname{Pr}_{P_{2}}\left[w\left(q_{t}\right) \mid \pi\right]=\frac{\left|\mathcal{G}_{2}\left(\pi \circ\left(q_{t}, w\left(q_{t}\right)\right)\right)\right|}{\left|\mathcal{G}_{2}(\pi)\right|} \leq \frac{\left|\mathcal{G}_{2}\left(\pi \circ\left(q_{t}, w\left(q_{t}\right)\right)\right)\right|}{\left|\mathcal{G}_{2}\left(\pi \circ\left(q_{t}, \overline{w\left(q_{t}\right)}\right)\right)\right|} .
$$

Therefore, to bound the probability that the algorithm observes a witness pair it is sufficient to bound the ratio between the number of graphs in $\mathcal{G}_{2}\left(\pi \circ\left(q, w\left(q_{t}\right)\right)\right)$ and the number of graphs in $\mathcal{G}_{2}\left(\pi \circ\left(q, \overline{w\left(q_{t}\right)}\right)\right)$. We do this by introducing an auxiliary graph, which is defined next. 


\subsubsection{The auxiliary graph for $t=m$}

For every $t \leq Q$, every query-answer history $\pi$ of length $t-1$ for which $\pi$ is consistent with $G_{1}$ (that is, no witness pair has yet been detected), and every pair $(u, v)$, we consider a bipartite auxiliary graph $\mathcal{A}_{\pi,(u, v)}$. On one side of $\mathcal{A}_{\pi,(u, v)}$ we have a node for every graph in $\mathcal{G}_{2}(\pi)$ for which the pair $(u, v)$ is a witness pair. We refer to these nodes as witness graphs. On the other side of the auxiliary graph, we place a node for every graph in $\mathcal{G}_{2}(\pi)$ for which the pair is not a witness. We refer to these nodes as non-witness graphs. We put an edge in the auxiliary graph between a witness graph $W$ and a non-witness graph $\bar{W}$ if the pair $(u, v)$ is a crossing (non-crossing) pair and the two graphs are identical except that their red (blue) matchings differ on exactly two pairs $-(u, v)$ and one additional pair. In other words, $\bar{W}$ can be obtained from $W$ by performing a switch operation, as defined next.

Definition 7. We define a switch between pairs in a matching in the following manner. Let $(u, v)$ and $\left(u^{\prime}, v^{\prime}\right)$ be two matched pairs in a matching $M . A$ switch between $(u, v)$ and $\left(u^{\prime}, v^{\prime}\right)$ means removing the edges $(u, v)$ and $\left(u^{\prime}, v^{\prime}\right)$ from $M$ and adding to it the edges $\left(u, v^{\prime}\right)$ and $\left(u^{\prime}, v\right)$.

Note that the switch process maintains the cardinality of the matching. We denote by $d_{w}\left(\mathcal{A}_{\pi,(u, v)}\right)$ the minimal degree of any witness graph in $\mathcal{A}_{\pi,(u, v)}$, and by $d_{n w}\left(\mathcal{A}_{\pi,(u, v)}\right)$ the maximal degree of the non-witness graphs. See Figure 3 for an illustration.

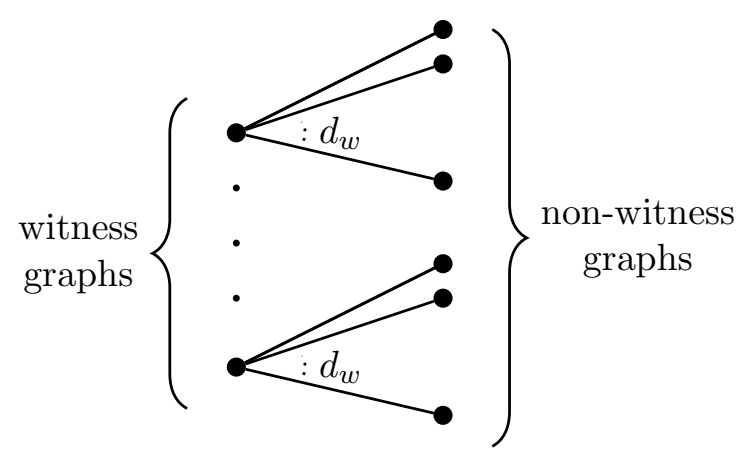

(a) The auxiliary graph with witness nodes on the left and non-witness nodes on the right.

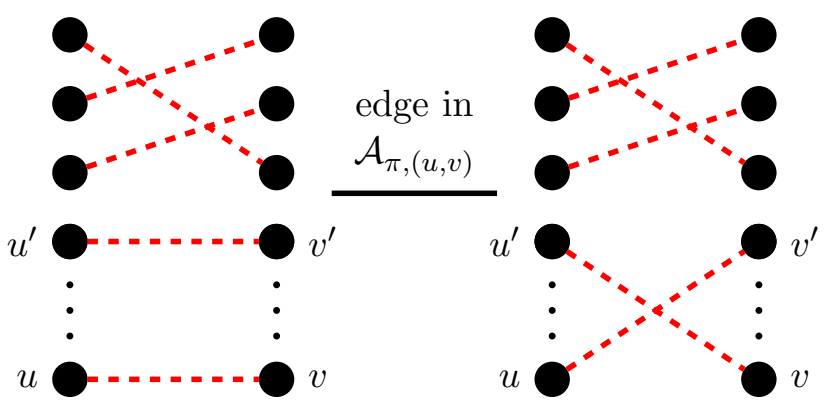

A witness graph $W \quad \bar{W}-$ A neighbor of $W$

(b) An illustration of two neighbors in the auxiliary graph for $t=m$.

Figure 3

Lemma 18. Let $t=m$ and $Q=\frac{m^{3 / 2}}{100 t}$. For every $t \leq Q$, every query-answer history $\pi$ of length $t-1$ such that $\pi$ is consistent with $G_{1}$ and every pair $(u, v)$,

$$
\frac{d_{n w}\left(\mathcal{A}_{\pi,(u, v)}\right)}{d_{w}\left(\mathcal{A}_{\pi,(u, v)}\right)} \leq \frac{2}{\sqrt{m}}=\frac{2 t}{m^{3 / 2}} .
$$

Proof. Recall that the graphs in $\mathcal{G}_{2}$ are as defined in Subsection 4.1.1 and illustrated in Figure 2. In the following we consider crossing pairs, as the proof for non-crossing pairs is almost identical. Recall that a crossing pair is a pair $(u, v)$ such that $u \in L$ and $v \in R$ or vise versa. A witness graph $W$ with respect to the pair $(u, v)$ is a graph in which $(u, v)$ is a red pair, i.e., $(u, v) \in M^{C}$. There is an edge from $W$ to every non-witness graph $\bar{W} \in \mathcal{G}_{2}(\pi)$ such that $M^{C}(W)$ and $M^{C}(\bar{W})$ differ exactly on $(u, v)$ and one additional edge. 
Every red pair $\left(u^{\prime}, v^{\prime}\right) \in M^{C}(W)$ creates a potential non-witness graph $\bar{W}_{\left(u^{\prime}, v^{\prime}\right)}$ when switched with $(u, v)$ (as defined in Definition 7). However, not all of the these non-witness graphs are in $\mathcal{G}_{2}(\pi)$. If $u^{\prime}$ is a neighbor of $v$ in the knowledge graph $G_{\pi}^{k n}$, i.e., $u^{\prime} \in \Gamma_{\pi}^{k n}(v)$, then $\bar{W}_{\left(u^{\prime}, v^{\prime}\right)}$ is not consistent with the knowledge graph, and therefore $\bar{W}_{\left(u^{\prime}, v^{\prime}\right)} \notin \mathcal{G}_{2}(\pi)$. This is also the case for a pair $\left(u^{\prime}, v^{\prime}\right)$ such that $v^{\prime} \in \Gamma_{\pi}^{k n}(u)$. Therefore, only pairs $\left(u^{\prime}, v^{\prime}\right) \in M^{C}$ such that $u^{\prime} \notin \Gamma_{\pi}^{k n}(v)$ and $v^{\prime} \notin \Gamma_{\pi}^{k n}(u)$ produce a non-witness graph $\bar{W}_{\left(u^{\prime}, v^{\prime}\right)} \in \mathcal{G}_{2}(\pi)$ when switched with $(u, v)$. We refer to these pairs as consistent pairs. Since $t \leq \frac{\sqrt{m}}{100}$, both $u$ and $v$ each have at most $\frac{m}{100}$ neighbors in the knowledge graph, implying that out of the $\sqrt{m}-1$ potential pairs, the number of consistent pairs is at least

$$
\sqrt{m}-1-d_{\pi}^{k n}(u)-d_{\pi}^{k n}(v) \geq \sqrt{m}-1-2 \cdot \frac{\sqrt{m}}{100} \geq \frac{1}{2} \sqrt{m}
$$

Therefore, the degree of every witness graph $W \in \mathcal{A}_{\pi,(u, v)}$ is at least $\frac{1}{2} \sqrt{m}$, implying that $d_{w}\left(\mathcal{A}_{\pi,(u, v)}\right) \geq \frac{1}{2} \sqrt{m}$.

In order to prove that $d_{n w}\left(\mathcal{A}_{\pi,(u, v)}\right)=1$, consider a non-witness graph $\bar{W}$. Since $\bar{W}$ is a nonwitness graph, the pair $(u, v)$ is not a red pair. This implies that $u$ is matched to some vertex $v^{\prime} \in R$, and $v$ is matched to some vertex $u^{\prime} \in L$. That is, $\left(u, v^{\prime}\right),\left(v, u^{\prime}\right) \in M^{C}$. By the construction of the edges in the auxiliary graph, every neighbor $W$ of $\bar{W}$ can be obtained by a single switch between two red pairs in the red matching. The only possibility to switch two pairs in $M^{C}(\bar{W})$ and obtain a matching in which $(u, v)$ is a red pair is to switch the pairs $\left(u, v^{\prime}\right)$ and $\left(v, u^{\prime}\right)$. Hence, every non-witness graph $\bar{W}$ has at most one neighbor.

We showed that $d_{w}\left(\mathcal{A}_{\pi,(u, v)}\right) \geq \frac{1}{2} \sqrt{m}$ and that $d_{n w}\left(\mathcal{A}_{\pi,(u, v)}\right) \leq 1$, implying

$$
\frac{d_{n w}\left(\mathcal{A}_{\pi,(u, v)}\right)}{d_{w}\left(\mathcal{A}_{\pi,(u, v)}\right)} \leq \frac{2}{\sqrt{m}}=\frac{2 t}{m^{3 / 2}}
$$

and the proof is complete.

\subsubsection{Statistical distance}

For a query-answer history $\pi$ of length $t-1$ and a query $q_{t}$, let $\operatorname{Ans}\left(\pi, q_{t}\right)$ denote the set of possible answers to the query $q_{t}$ that are consistent with $\pi$. Namely, if $q_{t}$ is a pair query (for a pair that does not belong to the knowledge graph $\left.G_{\pi}^{k n}\right)$, then $\operatorname{Ans}\left(\pi, q_{t}\right)=\{0,1\}$, and if $q_{t}$ is a random new-neighbor query, then $A n s\left(\pi, q_{t}\right)$ consists of all vertices except those in $N_{\pi}^{k n}$.

Lemma 19. Let $t=m$ and $Q=\frac{m^{3 / 2}}{100 t}$. For every $t \leq Q$, every query-answer history $\pi$ of length $t-1$ such that $\pi$ is consistent with $G_{1}$ and for every query $q_{t}$ :

$$
\sum_{a \in A n s\left(\pi, q_{t}\right)}\left|\operatorname{Pr}_{P_{1}}\left[a \mid \pi, q_{t}\right]-\operatorname{Pr}_{P_{2}}\left[a \mid \pi, q_{t}\right]\right| \leq \frac{12}{\sqrt{m}}=\frac{12 t}{m^{3 / 2}} .
$$

Proof. We prove the lemma separately for each type of query.

- We start with a crossing pair query $\left(u_{t}, v_{t}\right)$. In this case the witnesses are red pairs. Namely, our witness graphs for this case are all the graphs in $\mathcal{G}_{2}\left(\pi \circ\left(q_{t}, 0\right)\right)$, and the non-witness graphs are all the graphs in $\mathcal{G}_{2}\left(\pi \circ\left(q_{t}, 1\right)\right)$. By the construction of the auxiliary graph

$$
\left|\mathcal{G}_{2}\left(\pi \circ\left(q_{t}, 0\right)\right)\right| \cdot d_{w}\left(\mathcal{A}_{\pi,(u, v)}\right) \leq\left|\mathcal{G}_{2}\left(\pi \circ\left(q_{t}, 1\right)\right)\right| \cdot d_{n w}\left(\mathcal{A}_{\pi,(u, v)}\right) .
$$

This, together with Lemma 18, implies

$$
\frac{\left|\mathcal{G}_{2}\left(\pi \circ\left(q_{t}, 0\right)\right)\right|}{\left|\mathcal{G}_{2}(\pi)\right|} \leq \frac{\left|\mathcal{G}_{2}\left(\pi \circ\left(q_{t}, 0\right)\right)\right|}{\left|\mathcal{G}_{2}\left(\pi \circ\left(q_{t}, 1\right)\right)\right|} \leq \frac{d_{n w}\left(\mathcal{A}_{\pi,(u, v)}\right)}{d_{w}\left(\mathcal{A}_{\pi,(u, v)}\right)}=\frac{2}{\sqrt{m}}=\frac{2 t}{m^{3 / 2}}
$$


For a pair query $q_{t}$, the set of possible answers $\operatorname{Ans}\left(\pi, q_{t}\right)$ is $\{0,1\}$. Therefore,

$$
\begin{aligned}
\sum_{a \in\{0,1\}} & \left|\operatorname{Pr}_{P_{1}}\left[a \mid \pi, q_{t}\right]-\operatorname{Pr}_{P_{2}}\left[a \mid \pi, q_{t}\right]\right| \\
& =\left|\operatorname{Pr}_{P_{1}}\left[0 \mid \pi, q_{t}\right]-\operatorname{Pr}_{P_{2}}\left[0 \mid \pi, q_{t}\right]\right|+\left|\operatorname{Pr}_{P_{1}}\left[1 \mid \pi, q_{t}\right]-\operatorname{Pr}_{P_{2}}\left[1 \mid \pi, q_{t}\right]\right| \\
& =\frac{2 t}{m^{3 / 2}}+1-\left(1-\frac{2 t}{m^{3 / 2}}\right)=\frac{4 t}{m^{3 / 2}}=\frac{4}{\sqrt{m}} .
\end{aligned}
$$

- For a non-crossing pair query $q_{t}=(u, v)$ our witness graphs are graphs that contain $q_{t}$ as a blue pair, i.e., graphs from $\mathcal{G}_{2}\left(\pi,\left(q_{t}, 1\right)\right)$, and our non-witness graphs are graphs in which no blue pair had been queried, i.e., graphs from $\mathcal{G}_{2}\left(\pi,\left(q_{t}, 0\right)\right)$. From Lemma 18 we get that for a non-crossing pair query $q_{t}$ :

$$
\frac{\left|\mathcal{G}_{2}\left(\pi \circ\left(q_{t}, 1\right)\right)\right|}{\left|\mathcal{G}_{2}(\pi)\right|} \leq \frac{\left|\mathcal{G}_{2}\left(\pi \circ\left(q_{t}, 1\right)\right)\right|}{\left|\mathcal{G}_{2}\left(\pi \circ\left(q_{t}, 0\right)\right)\right|} \leq \frac{d_{n w}\left(\mathcal{A}_{\pi,(u, v)}\right)}{d_{w}\left(\mathcal{A}_{\pi,(u, v)}\right)}=\frac{2 t}{m^{3 / 2}}=\frac{2}{\sqrt{m}} .
$$

Therefore,

$$
\begin{aligned}
& \sum_{a \in\{0,1\}}\left|\operatorname{Pr}_{P_{1}}\left[a \mid \pi, q_{t}\right]-\operatorname{Pr}_{P_{2}}\left[a \mid \pi, q_{t}\right]\right| \\
& \quad=\left|\operatorname{Pr}_{P_{1}}\left[0 \mid \pi, q_{t}\right]-\operatorname{Pr}_{P_{2}}\left[0 \mid \pi, q_{t}\right]\right|+\left|\operatorname{Pr}_{P_{1}}\left[1 \mid \pi, q_{t}\right]-\operatorname{Pr}_{P_{2}}\left[1 \mid \pi, q_{t}\right]\right| \\
& \quad=1-\left(1-\frac{2 t}{m^{3 / 2}}\right)+\frac{2 t}{m^{3 / 2}}=\frac{4 t}{m^{3 / 2}}=\frac{4}{\sqrt{m}} .
\end{aligned}
$$

- For a new-neighbor query $q_{t}=u_{t}$, the set of possible answers $\operatorname{Ans}\left(\pi, q_{t}\right)$ is the set of all the vertices in the graph. Therefore,

$$
\begin{aligned}
& \sum_{a \in \operatorname{Ans}\left(\pi, q_{t}\right)}\left|\operatorname{Pr}_{P_{1}}\left[a \mid \pi, q_{t}\right]-\operatorname{Pr}_{P_{2}}\left[a \mid \pi, q_{t}\right]\right| \\
= & \sum_{v \in R}\left|\operatorname{Pr}_{P_{1}}\left[v \mid \pi, q_{t}\right]-\operatorname{Pr}_{P_{2}}\left[v \mid \pi, q_{t}\right]\right|+\sum_{v \in L}\left|\operatorname{Pr}_{P_{1}}\left[v \mid \pi, q_{t}\right]-\operatorname{Pr}_{P_{2}}\left[v \mid \pi, q_{t}\right]\right| .
\end{aligned}
$$

Recall that for a vertex $v \in \Gamma_{\pi}^{k n}(u), \operatorname{Pr}_{P_{1}}\left[v \mid \pi, q_{t}\right]=\operatorname{Pr}_{P_{2}}\left[v \mid \pi, q_{t}\right]=0$. Therefore, it suffices to consider only vertices $v$ such that $v \notin \Gamma_{\pi}^{k n}(u)$. Assume without loss of generality that $u \in L$, and consider a vertex $v \in R, v \notin \Gamma_{\pi}^{k n}(u)$. Since for every $v \in R$ we have that $\left(u_{t}, v\right) \in E\left(G_{1}\right)$, by the definition of $P_{1}$,

$$
\operatorname{Pr}_{P_{1}}\left[v \mid \pi, q_{t}\right]=\frac{1}{\sqrt{m}-d_{\pi}^{k n}\left(u_{t}\right)} .
$$

Now consider the process $P_{2}$. By its definition,

$$
\begin{aligned}
\operatorname{Pr}_{P_{2}}\left[v \mid \pi, q_{t}\right] & =\frac{\mathcal{G}_{2}\left(\pi \circ\left(q_{t}, v\right)\right)}{\mathcal{G}_{2}(\pi)} \cdot \frac{1}{\sqrt{m}-d_{\pi}^{k n}(u)} \\
& =\frac{\mathcal{G}_{2}(\pi \circ((u, v), 1))}{\mathcal{G}_{2}(\pi)} \cdot \frac{1}{\sqrt{m}-d_{\pi}^{k n}(u)} \\
& =\left(1-\frac{\mathcal{G}_{2}(\pi \circ((u, v), 0))}{\mathcal{G}_{2}(\pi)}\right) \cdot \frac{1}{\sqrt{m}-d_{\pi}^{k n}(u)} .
\end{aligned}
$$


By the first item in the proof, for any crossing pair $q_{t}=(u, v)$,

$$
\frac{\mathcal{G}_{2}\left(\pi \circ\left(q_{t}, 0\right)\right)}{\mathcal{G}_{2}(\pi)}=\frac{4 t}{m^{3 / 2}}=\frac{4}{\sqrt{m}},
$$

and it follows that

$$
\operatorname{Pr}_{P_{2}}\left[v \mid \pi, q_{t}\right]=\left(1-\frac{4 t}{m^{3 / 2}}\right) \cdot \frac{1}{\sqrt{m}-d_{\pi}^{k n}(u)} .
$$

By Equations (15) and (16), we get that for every $v \in R$ such that $v \notin \Gamma_{\pi}^{k n}(u)$,

$$
\left|\operatorname{Pr}_{P_{1}}\left[v \mid \pi, q_{t}\right]-\operatorname{Pr}_{P_{2}}\left[v \mid \pi, q_{t}\right]\right|=\frac{4 t / m^{3 / 2}}{\sqrt{m}-d_{\pi}^{k n}(u)} .
$$

Therefore,

$$
\begin{gathered}
\sum_{v \in R}\left|\operatorname{Pr}_{P_{1}}\left[v \mid \pi, q_{t}\right]-\operatorname{Pr}_{P_{2}}\left[v \mid \pi, q_{t}\right]\right|=\sum_{v \in R, v \notin \Gamma_{\pi}^{k n}(u)}\left|\operatorname{Pr}_{P_{1}}\left[v \mid \pi, q_{t}\right]-\operatorname{Pr}_{P_{2}}\left[v \mid \pi, q_{t}\right]\right| \\
=\left(\sqrt{m}-d_{\pi}^{k n}(u)\right) \cdot \frac{4 t / m^{3 / 2}}{\sqrt{m}-d_{\pi}^{k n}(u)}=\frac{4 t}{m^{3 / 2}}=\frac{4}{\sqrt{m}} .
\end{gathered}
$$

Now consider a vertex $v \in L$. Observe that for every $v \in L$, it holds that $v \notin \Gamma_{\pi}^{k n}(u)$ since otherwise $\pi$ is not consistent with $G_{1}$. For the same reason,

$$
\operatorname{Pr}_{P_{1}}\left[v \mid \pi, q_{t}\right]=0 \text {. }
$$

As for $P_{2}$, as before,

$$
\operatorname{Pr}_{P_{2}}\left[v \mid \pi, q_{t}\right]=\frac{\mathcal{G}_{2}\left(\pi,\left(u_{t}, v\right)\right)}{\mathcal{G}_{2}(\pi)} \cdot \frac{1}{\sqrt{m}-d_{\pi}^{k n}\left(u_{t}\right)} .
$$

By the second item of the claim, since for every $v \in L,\left(u_{t}, v\right)$ is a non-crossing pair, we have that

$$
\frac{\left|\mathcal{G}_{2}\left(\pi,\left(u_{t}, v\right)\right)\right|}{\left|\mathcal{G}_{2}(\pi)\right|}=\frac{4 t}{m^{3 / 2}}=\frac{4}{\sqrt{m}} .
$$

Combining Equations (19) and (20) we get that for every $v \in L$

$$
\left|\operatorname{Pr}_{P_{1}}\left[v \mid \pi, q_{t}\right]-\operatorname{Pr}_{P_{2}}\left[v \mid \pi, q_{t}\right]\right|=\frac{4 t / m^{3 / 2}}{\sqrt{m}-d_{\pi}^{k n}(u)} .
$$

Since $Q=\frac{m^{3 / 2}}{100 t}=\frac{\sqrt{m}}{100}$, for every $t \leq Q, d_{\pi}^{k n}(u)<\frac{1}{2} \sqrt{m}$, and it follows that $\frac{\sqrt{m}-1}{\sqrt{m}-d^{k n}(u)}$ is bounded by 2 . Hence,

$$
\begin{aligned}
\sum_{v \in L}\left|\operatorname{Pr}_{P_{1}}\left[v \mid \pi, q_{t}\right]-\operatorname{Pr}_{P_{2}}\left[v \mid \pi, q_{t}\right]\right| & =(\sqrt{m}-1) \cdot \frac{4 t / m^{3 / 2}}{\sqrt{m}-d_{\pi}^{k n}(u)} \\
& =\frac{8 t}{m^{3 / 2}}=\frac{8}{\sqrt{m}} .
\end{aligned}
$$

By Equations (18) and (21) we get

$$
\begin{aligned}
& \sum_{v \in R}\left|\operatorname{Pr}_{P_{1}}\left[v \mid \pi, q_{t}\right]-\operatorname{Pr}_{P_{2}}\left[v \mid \pi, q_{t}\right]\right|+\sum_{v \in L}\left|\operatorname{Pr}_{P_{1}}\left[v \mid \pi, q_{t}\right]-\operatorname{Pr}_{P_{2}}\left[v \mid \pi, q_{t}\right]\right| \\
& \quad=\frac{12 t}{m^{3 / 2}}=\frac{12}{\sqrt{m}} .
\end{aligned}
$$


This completes the proof.

Recall that $\mathcal{D}_{b}^{\mathrm{ALG}}, b \in\{1,2\}$, denotes the distribution on query-answer histories of length $Q$, induced by the interaction of ALG and $P_{b}$. We show that the two distributions are indistinguishable for $Q$ that is sufficiently small.

Lemma 20. Let $t=m$. For every algorithm $A L G$ that asks at most $Q=\frac{m^{3 / 2}}{100 t}$ queries, the statistical distance between $\mathcal{D}_{1}^{A L G}$ and $\mathcal{D}_{2}^{A L G}$ is at most $\frac{1}{3}$.

Proof. Consider the following hybrid distribution. Let $\mathcal{D}_{1, t}^{\mathrm{ALG}}$ be the distribution over query-answer histories of length $Q$, where in the length $t$ prefix ALG is answered by the process $P_{1}$ and in the length $Q-t$ suffix ALG is answered by the process $P_{2}$. Observe that $\mathcal{D}_{1, Q}^{\mathrm{ALG}}=\mathcal{D}_{1}^{\mathrm{ALG}}$ and that $\mathcal{D}_{1,0}^{\mathrm{ALG}}=\mathcal{D}_{2}^{\mathrm{ALG}}$. Let $\pi=\left(\pi_{1}, \pi_{2}, \ldots, \pi_{\ell}\right)$ denote a query-answer history of length $\ell$. By the triangle inequality $d\left(\mathcal{D}_{1}^{\mathrm{ALG}}, \mathcal{D}_{2}^{\mathrm{ALG}}\right) \leq \sum_{t=0}^{Q-1} d\left(\mathcal{D}_{1, t+1}^{\mathrm{ALG}}, \mathcal{D}_{1, t}^{\mathrm{ALG}}\right)$

$$
d\left(\mathcal{D}_{1}^{\mathrm{ALG}}, \mathcal{D}_{2}^{\mathrm{ALG}}\right) \leq \sum_{t=0}^{Q-1} d\left(\mathcal{D}_{1, t+1}^{\mathrm{ALG}}, \mathcal{D}_{1, t}^{\mathrm{ALG}}\right) .
$$

It thus remains to bound $d\left(\mathcal{D}_{1, t+1}^{\mathrm{ALG}}, \mathcal{D}_{1, t}^{\mathrm{ALG}}\right)=\frac{1}{2} \sum_{\pi}\left|\operatorname{Pr}_{\mathcal{D}_{1, t+1}^{\mathrm{ALG}}}[\pi]-\operatorname{Pr}_{\mathcal{D}_{1, t}^{\mathrm{ALG}}}[\pi]\right|$ for every $t$ such that $0 \leq t \leq Q-1$. Let $\mathcal{Q}$ denote the set of all possible queries.

$$
\begin{aligned}
\sum_{\pi} \mid \operatorname{Pr}_{\mathcal{D}_{1, t+1}^{\mathrm{ALG}}}[\pi]-\operatorname{Pr}_{\mathcal{D}_{1, t}^{\mathrm{ALG}}[\pi] \mid}= & \sum_{\pi_{1}, \ldots, \pi_{t-1}} \operatorname{Pr}_{P_{1}, \mathrm{ALG}}\left[\pi_{1}, \ldots, \pi_{t-1}\right] \cdot \sum_{q \in \mathcal{Q}} \operatorname{Pr}_{\mathrm{ALG}}\left[q \mid \pi_{1}, \ldots, \pi_{t-1}\right] \\
\cdot & \sum_{a \in A n s\left(\left(\pi_{1}, \ldots, \pi_{t-1}\right), q\right)}\left|\operatorname{Pr}_{P_{1}}\left[a \mid \pi_{1}, \ldots, \pi_{t-1}, q\right]-\operatorname{Pr}_{P_{2}}\left[a \mid \pi_{1}, \ldots, \pi_{t-1}, q\right]\right| \\
& \sum_{\pi_{t+1}, \ldots, \pi_{Q}} \operatorname{Pr}_{P_{2}, \mathrm{ALG}}\left[\pi_{t+1}, \ldots, \pi_{Q} \mid \pi_{1}, \ldots, \pi_{t-1},(q, a)\right] .
\end{aligned}
$$

By Lemma 19 , for every $1 \leq t \leq Q-1$, and every $\pi_{1}, \ldots, \pi_{t-1}$ and $q$,

$$
\sum_{a \in \operatorname{Ans}\left(\left(\pi_{1}, \ldots, \pi_{t-1}\right), q\right)}\left|\operatorname{Pr}_{P_{1}}\left[a \mid \pi_{1}, \ldots, \pi_{t-1}, q\right]-\operatorname{Pr}_{P_{2}}\left[a \mid \pi_{1}, \ldots, \pi_{t-1}, q\right]\right| \leq \frac{12 t}{m^{3 / 2}} .
$$

We also have that for every pair $(q, a)$,

$$
\sum_{\pi_{t+1}, \ldots, \pi_{Q}} \operatorname{Pr}_{P_{2}, \mathrm{ALG}}\left[\pi_{t+1}, \ldots, \pi_{Q} \mid \pi_{1}, \ldots, \pi_{t-1},(q, a)\right]=1 .
$$

Therefore,

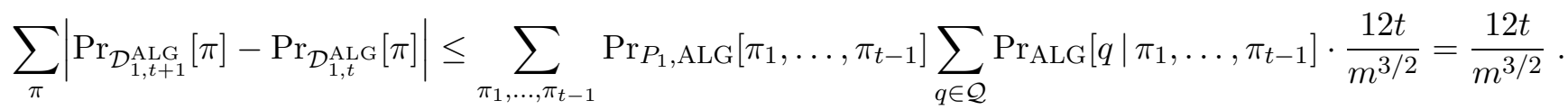

Hence, for $Q=\frac{\sqrt{m}}{100}$,

$$
d\left(\mathcal{D}_{1}^{\mathrm{ALG}}, \mathcal{D}_{2}^{\mathrm{ALG}}\right)=\frac{1}{2} \sum_{\pi} \sum_{t=1}^{Q-1}\left|\operatorname{Pr}_{\mathcal{D}_{1, t+1}^{\mathrm{ALG}}}[\pi]-\operatorname{Pr}_{\mathcal{D}_{1, t}^{\mathrm{ALG}}}[\pi]\right| \leq \frac{1}{2} \cdot Q \cdot \frac{12 t}{m^{3 / 2}} \leq \frac{1}{3},
$$

and the proof is complete. 
In the next subsection we turn to prove the theorem for the cases where $m<t \leq \frac{m^{3 / 2}}{8}$, and for the case where $\sqrt{m} \leq t \leq \frac{m}{4}$. We start with the former case. The proof will follow the building blocks of the proof for $t=m$, where the only difference is in the description of the auxiliary graph $\mathcal{A}_{\pi,(u, v)}$ and in the proof that $\frac{d_{n w}\left(\mathcal{A}_{\pi,(u, v)}\right)}{d_{w}\left(\mathcal{A}_{\pi,(u, v)}\right)} \leq \frac{2 t}{m^{3 / 2}}=\frac{2 r}{\sqrt{m}}$.

\subsection{A lower bound for $m<t<m^{3 / 2}$}

Let $t=r \cdot m$ for an integer $r$ such that $1<r \leq \frac{1}{8} \sqrt{m}$. It is sufficient for our needs to consider only values of $t$ for which $r$ is an integer. The proof of the lower bound for this case is a fairly simple extension of the proof for the case of $t=m$, that is, $r=1$. We next describe the modifications we make in the construction of $\mathcal{G}_{2}$.

\subsubsection{The lower-bound construction}

Let $G_{1}$ be as defined in Subsection 4.1.1. The construction of $\mathcal{G}_{2}$ for $t=r \cdot m$ can be thought of as repeating the construction of $\mathcal{G}_{2}$ for $t=m$ (as described in Subsection 4.1.1) $r$ times. We again start with a complete bipartite graph $K_{\sqrt{m}, \sqrt{m}}$ and an independent set of size $n-2 \sqrt{m}$. For each graph $G \in \mathcal{G}_{2}$ we select $r$ perfect matchings between the two sides $R$ and $L$ and remove these edges from the graph. We denote the $r$ perfect matchings by $M_{1}^{C}, \ldots, M_{r}^{C}$ and refer to them as the red matchings. We require that each two perfect matchings $M_{i}^{C}$ and $M_{j}^{C}$ do not have any shared edges. That is, for every $i$ and for every $j$, for every $(u, v) \in M_{i}^{C}$ it holds that $(u, v) \notin M_{j}^{C}$. In order to maintain the degrees of the vertices, we next select $r$ perfect matchings for each side of the bipartite graph ( $L$ to $L$ and $R$ to $R$ ). We denote these matchings by $M_{1}^{R}, \ldots, M_{r}^{R}$ and $M_{1}^{L}, \ldots, M_{r}^{L}$ respectively. Again we require that no two matchings share an edge. We refer to these matchings as the blue matchings and their edges as blue pairs. Each such choice of $3 r$ matchings defines a graph in $\mathcal{G}_{2}$.

Let $G$ be a graph in $\mathcal{G}_{2}$. We say that a triangle is blue if all its edges are blue. Otherwise we say the triangle is mixed. Observe that every blue edge in $G$ participates in at least $\sqrt{m}-2 r$ mixed triangles, and at most $r$ blue triangles. Also note that every two mixed triangles are disjoint. Therefore, there are at least $\frac{1}{2} r \sqrt{m} \cdot(2 \sqrt{m}-2 r)=\Omega(r \cdot m)$ and at most $\frac{1}{2} r \sqrt{m} \cdot(2 \sqrt{m}-2 r)+r^{2} \sqrt{m}$ triangles in $G$. Since $r<\frac{1}{8} \sqrt{m}$, we get that every graph in $G$ has $\Theta(r \cdot m)$ triangles.

\subsubsection{The processes $P_{1}$ and $P_{2}$}

The definition of the processes $P_{1}$ and $P_{2}$ is the same as in Subsection 4.1.2 (using the modified definition of $\mathcal{G}_{2}$ ), and Lemma 17 holds here as well.

\subsubsection{The auxiliary graph}

As before, for every $t \leq Q$, every query-answer history $\pi$ of length $t-1$ such that $\pi$ is consistent with $G_{1}$ and every pair $(u, v)$, we define a bipartite auxiliary graph $\mathcal{A}_{\pi,(u, v)}$, such that on one side there is a node for every witness graph $W \in \mathcal{G}_{2}(\pi)$, and on the other side a node for every non-witness graph $\bar{W} \in \mathcal{G}_{2}(\pi)$. The witness graphs for this case are graphs in which $(u, v)$ is a red (blue) edge in one of the red (blue) matchings. If $(u, v)$ is a crossing pair, then for every witness graph $W$, $(u, v) \in M_{i}^{C}(W)$ for some $1 \leq i \leq r$. If $(u, v)$ is a non-crossing pair, then for every witness graph $W,(u, v) \in M_{i}^{L}(W)$ or $(u, v) \in M_{i}^{L}(W)$. There is an edge from $W$ to every graph $\bar{W}$ such that the matching that contains $(u, v)$ in $W$ and the corresponding matching in $\bar{W}$ differ on exactly two pairs $-(u, v)$ and one additional pair. For example, if $(u, v) \in M_{i}^{C}(W)$, there is an edge from $W$ to every graph $\bar{W}$ such that $M_{i}^{C}(W)$ and $M_{i}^{C}(\bar{W})$ differ on exactly $(u, v)$ and one additional pair. 
Lemma 21. Let $t=r \cdot m$ for an integer $r$ such that $1<r \leq \frac{\sqrt{m}}{8}$ and let $Q=\frac{m^{3 / 2}}{100 t}$. For every $t \leq Q$, every query-answer history $\pi$ of length $t-1$ such that $\pi$ is consistent with $G_{1}$ and every pair $(u, v)$,

$$
\frac{d_{n w}\left(\mathcal{A}_{\pi,(u, v)}\right)}{d_{w}\left(\mathcal{A}_{\pi,(u, v)}\right)} \leq \frac{2 t}{m^{3 / 2}}=\frac{2 r}{\sqrt{m}} .
$$

Proof. We again analyze the case in which the pair is a crossing pair $(u, v)$, as the proof for a non-crossing pair is almost identical. We first consider the minimal degree of the witness graphs in $\mathcal{A}_{\pi,(u, v)}$. Let $M_{i}^{C}$ be the matching to which $(u, v)$ belongs. As before, only pairs $\left(u^{\prime}, v^{\prime}\right) \in M_{i}^{C}$ such that $u^{\prime} \notin \Gamma_{\pi}^{k n}(u), v^{\prime} \notin \Gamma_{\pi}^{k n}(v)$ result in a non-witness graph $\bar{W} \in \mathcal{G}_{2}(\pi)$ when switched with $(u, v)$. However, we have an additional constraint. Since by our construction no two red matchings share an edge, it must be that $u^{\prime}$ is not matched to $v$ in any of the other $r$ red matching, and similarly that $u$ is not matched to $v^{\prime}$ in any of the other matchings. It follows that of the $\left(\sqrt{m}-1-2 \cdot \frac{m^{3 / 2}}{100 \cdot r \cdot m}\right)$ potential pairs (as in the proof of Lemma 18), we discard $2 r$ additional pairs. Since $1 \leq r \leq \frac{\sqrt{m}}{8}$ we remain with $\left(\sqrt{m}-1-\frac{\sqrt{m}}{50}-\frac{1}{4} \sqrt{m}\right) \geq \frac{1}{2} \sqrt{m}$ potential pairs. Thus, $d_{w}\left(\mathcal{A}_{\pi,(u, v)}\right) \geq \frac{1}{2} \sqrt{m}$.

We now turn to consider the degree of the non-witness graphs and prove that $d_{n w}\left(\mathcal{A}_{\pi,(u, v)}\right) \leq r$. Consider a non-witness graph $\bar{W}$. To prove that $\bar{W}$ has at most $r$ neighbors it is easier to consider all the possible options to "turn" $\bar{W}$ from a non-witness graph into a witness graph. It holds that for every $j \in[r],(u, v) \notin M_{j}^{C}(\bar{W})$. Therefore for every matching $M_{j}^{C}, u$ is matched to some vertex, denoted $v_{j}^{\prime}$ and $v$ is matched to some vertex, denoted $u_{j}^{\prime}$. If we switch between the pairs $\left(u, v_{j}^{\prime}\right)$ and $\left(v, u_{j}^{\prime}\right)$, this results in a matching in which $(u, v)$ is a witness pair. We again refer the reader to Figure $3 \mathrm{~b}$, where the illustrated matching can be thought of as the $j^{\text {th }}$ matching. Denote the resulting graph by $W_{\left(u_{j}^{\prime}, v_{j}^{\prime}\right)}$. If the pair $\left(u_{j}^{\prime}, v_{j}^{\prime}\right)$ has not been observed yet by the algorithm then $W_{\left(u_{j}^{\prime}, v_{j}^{\prime}\right)}$ is a witness graph in $\mathcal{A}_{\pi,(u, v)}$. Therefore there are at most $r$ options to turn $\bar{W}$ into a witness graph, and $d_{n w}\left(\mathcal{A}_{\pi,(u, v)}\right) \leq r$. We showed that $d_{w}\left(\mathcal{A}_{\pi,(u, v)}\right) \geq \frac{1}{2} \sqrt{m}$ and $d_{n w}\left(\mathcal{A}_{\pi,(u, v)}\right) \leq r$, implying

$$
\frac{d_{n w}\left(\mathcal{A}_{\pi,(u, v)}\right)}{d_{w}\left(\mathcal{A}_{\pi,(u, v)}\right)} \leq \frac{2 r}{\sqrt{m}}=\frac{2 t}{m^{3 / 2}},
$$

as required.

\subsubsection{Statistical distance}

The proof of the next lemma is exactly the same as the proof of Lemma 19, except that occurrences of the term $\left(t / m^{3 / 2}\right)$ are replaced by $(r / \sqrt{m})$ instead of $(1 / \sqrt{m})$, and we apply Lemma 21 instead of Lemma 18.

Lemma 22. Let $t=r \cdot m$ for an integer $r$ such that $1<r \leq \frac{\sqrt{m}}{8}$ and let $Q=\frac{m^{3 / 2}}{100 t}$. For every $t \leq Q$, every query-answer history $\pi$ of length $t-1$ such that $\pi$ is consistent with $G_{1}$ and for every query $q_{t}$,

$$
\sum_{a \in A n s\left(\pi, q_{t}\right)}\left|\operatorname{Pr}_{P_{1}}\left[a \mid \pi, q_{t}\right]-\operatorname{Pr}_{P_{2}}\left[a \mid \pi, q_{t}\right]\right|=\frac{12 t}{m^{3 / 2}}=\frac{12 r}{\sqrt{m}} .
$$

The proof of the next lemma is same as the proof of Lemma 20 except that we replace the application of Lemma 19, by an application of Lemma 22.

Lemma 23. Let $t=r \cdot m$ for an integer $r$ such that $1<r \leq \frac{\sqrt{m}}{8}$. For every algorithm ALG that performs at most $Q=\frac{m^{3 / 2}}{100 t}$ queries, the statistical distance between $\mathcal{D}_{1}^{A L G}$ and $\mathcal{D}_{2}^{A L G}$ is at most $\frac{1}{3}$. 


\subsection{A lower bound for $\sqrt{m} \leq t \leq \frac{1}{4} m$}

Similarly to the previous section, we let $t=k \sqrt{m}$ and assume that $k$ is an integer such that $1 \leq k \leq \frac{\sqrt{m}}{4}$.

\subsubsection{The lower-bound construction}

The construction of the graph $G_{1}$ is as defined in Subsection 4.1.1, and we modify the construction of the graphs in $\mathcal{G}_{2}$. As before, the basic structure of every graph is a complete bipartite graph $K_{\sqrt{m}, \sqrt{m}}$ and an independent set of size $n-2 \sqrt{m}$ vertices. In this case, for each graph in $\mathcal{G}_{2}$, we do not remove a perfect matching from the bipartite graph, but rather a matching $M^{C}$ of size $k$. In order to keep the degrees of all vertices to be $\sqrt{m}$, we modify the way we construct the blue matchings. Let $M^{C}=$ $\left\{\left(\ell_{i_{1}}, r_{i_{1}}\right),\left(\ell_{i_{2}}, r_{i_{2}}\right), \ldots,\left(\ell_{i_{k}}, r_{i_{k}}\right)\right\}$ be the crossing matching. The blue matchings will be $M^{L}=$ $\left\{\left(\ell_{i_{1}}, \ell_{i_{2}}\right),\left(\ell_{i_{3}}, \ell_{i_{4}}\right), \ldots,\left(\ell_{i_{k}-1}, \ell_{i_{k}}\right)\right\}$ and $M^{R}=\left\{\left(r_{i_{1}}, r_{i_{2}}\right),\left(r_{i_{3}}, r_{i_{4}}\right), \ldots,\left(r_{i_{k}-1}, r_{i_{k}}\right)\right\}$. Note that every matched pair belongs to a four-tuple $\left\langle\ell_{i_{j}}, \ell_{i_{j+1}}, r_{i_{j+1}}, r_{i_{j}}\right\rangle$ such that $\left(\ell_{i_{j}}, r_{i_{j}}\right)$ and $\left(\ell_{i_{j+1}}, r_{i_{j+1}}\right)$ are red pairs and $\left(\ell_{i_{j}}, \ell_{i_{j+1}}\right)$ and $\left(r_{i_{j}}, r_{i_{j+1}}\right)$ are blue pairs. We refer to these structures as matched squares and to four-tuples $\left(\ell_{x}, \ell_{y}, r_{z}, r_{w}\right)$ such that no pair in the tuple is matched as unmatched squares. See Figure 4 for an illustration. Every graph in $\mathcal{G}_{2}$ is defined by its set of $k$ four-tuples.

Similarly to previous constructions, in every graph $G \in \mathcal{G}_{2}$, every blue edge participates in $\sqrt{m}-2$ triangles. Since every triangle in the $G$ contains exactly one blue edge, we have that $G$ has $k \cdot(\sqrt{m}-2)=\Theta(k \sqrt{m})$ triangles.

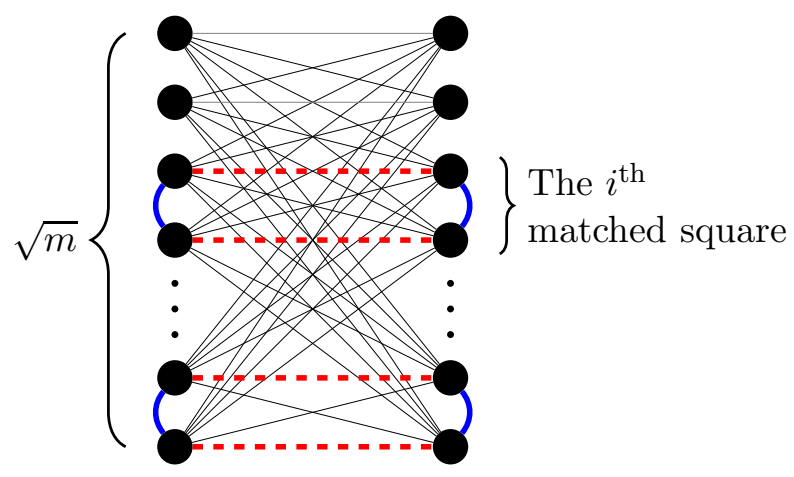

Figure 4: An illustration of the bipartite component in the family $\mathcal{G}_{2}$ for $\sqrt{m} \leq t \leq \frac{1}{4} m$.

\subsubsection{The processes $P_{1}$ and $P_{2}$}

We introduce a small modification to the definition of the processes $P_{1}$ and $P_{2}$. Namely, we leave the answering process for pair queries as described in Subsection 4.1.2 and modify the answering process for random new-neighbor queries as follows. Let $t \leq Q$, and $\pi$ be a query-answer history of length $t-1$ such that $\pi$ is consistent with $G_{1}$. If the $t^{\text {th }}$ query is a new-neighbor query $q_{t}=u$ and $d_{\pi}^{k n}(u)<\frac{1}{2} \sqrt{m}$, then the processes $P_{1}$ and $P_{2}$ answer as described in Subsection 4.1.2. However, if the $t^{\text {th }}$ query is a new-neighbor query $q_{t}=u$ such that $d_{\pi}^{k n}(u) \geq \frac{1}{2} \sqrt{m}$, then the processes answers as follows.

- The process $P_{1}$ answers with the set of all neighbors of $u$ in $G_{1}$. That is, if $u$ is in $L$, then the process replies with $a=R=\left\{r_{1}, \ldots, r_{\sqrt{m}}\right\}$, and if $u$ is in $R$, then the process replies with $a=L=\left\{\ell_{1}, \ldots, \ell_{\sqrt{m}}\right\}$. 
The process $P_{2}$ answers with $a=\left\{v_{1}, \ldots, v_{\sqrt{m}}\right\}$, where $\left\{v_{1}, \ldots, v_{\sqrt{m}}\right\}$ is the set of neighbors of $u$ in a subset of the graphs in $\mathcal{G}_{2}$. By the definition of $\mathcal{G}_{2}$, if $u$ is in $L$, then this set is either $R$, or it is $R \backslash\left\{r_{i}\right\} \cup\left\{\ell_{j}\right\}$ for some $r_{i} \in R$ and $\ell_{j} \in L$, and if $u$ is in $R$, then this set is either $L$, or it is $L \backslash\left\{\ell_{i}\right\} \cup\left\{r_{j}\right\}$ for some $\ell_{i} \in L$ and $r_{j} \in R$. For every such set $a \in \operatorname{Ans}\left(\pi, q_{t}\right)$, the process returns $a$ as an answer with probability

$$
\frac{\left|\mathcal{G}_{2}\left(\pi \circ\left(q_{t}, a\right)\right)\right|}{\left|\mathcal{G}_{2}(\pi)\right|} .
$$

We call this query an all-neighbors query.

First note that the above modification makes the algorithm "more powerful". That is, every algorithm that is not allowed all-neighbors query can be emulated by an algorithm that is allowed this type of query. Therefore this only strengthen our lower bound results.

Also note that this modification does not affect the correctness of Lemma 17. We can redefine the function $\alpha_{t}(\pi)$ to be

$$
\alpha_{t}(\pi)= \begin{cases}1 & \text { if } q_{t}(\pi) \text { is a pair query } \\ 1 /\left(\sqrt{m}-d_{\pi \leq t-1}^{k n}(u)\right) & \text { if } q_{t}(\pi)=u \text { is a random new-neighbor query } \\ 1 & \text { if } q_{t}(\pi) \text { is an all-neighbors query }\end{cases}
$$

and the rest of the proof follows as before.

\subsubsection{The auxiliary graph}

For every $t \leq Q$, every query-answer history $\pi$ of length $t-1$ such that $\pi$ is consistent with $G_{1}$ and every pair $(u, v)$, the witness graphs in $\mathcal{A}_{\pi,(u, v)}$ are graphs in which $(u, v)$ is either a red pair or a blue pair. There is an edge between a witness graph $W$ and a non-witness graph $\bar{W}$ if the two graphs have the same set of four-tuples except for two matched squares - one that contains the pair $(u, v),\left\langle u, v, u^{\prime}, v^{\prime}\right\rangle$ and another one.

Definition 8. We define a switch between a matched square and an unmatched square in the following manner. Let $\left\langle u, v, u^{\prime}, v^{\prime}\right\rangle$ be a matched square and $\left\langle x, y, x^{\prime}, y^{\prime}\right\rangle$ be an un matched squares. Informally, a switch between the squares is "unmatching" the matched square and instead "matching" the unmatched square.

Formally, a switch consists of two steps. The first step is removing the edges $(u, v)$ and $\left(u^{\prime}, v^{\prime}\right)$ from the red matching $M^{C}$ and the edges $\left(u, u^{\prime}\right)$ and $\left(v, v^{\prime}\right)$ from the blue matchings $M^{L}$ and $M^{R}$ respectively. The second step is adding the edges $(x, y)$ and $\left(x^{\prime}, y^{\prime}\right)$ from the red matching $M^{C}$ and the edges $\left(x, x^{\prime}\right)$ and $\left(y, y^{\prime}\right)$ from the blue matchings $M^{L}$ and $M^{R}$ respectively. See Figure 5 for an illustration.

Lemma 24. Let $t=k \cdot \sqrt{m}$ for an integer $k$ such that $1<k \leq \frac{\sqrt{m}}{4}$ and let $Q=\frac{m^{3 / 2}}{600 t}$. For every $t \leq Q$, every query-answer history $\pi$ of length $t-1$ such that $\pi$ is consistent with $G_{1}$ and every pair $(u, v)$,

$$
\frac{d_{n w}\left(\mathcal{A}_{\pi,(u, v)}\right)}{d_{w}\left(\mathcal{A}_{\pi,(u, v)}\right)}=\frac{16 k}{m}=\frac{16 t}{m^{3 / 2}} .
$$

Proof. We start with proving that $d_{w}\left(\mathcal{A}_{\pi,(u, v)}\right) \geq \frac{1}{2} m$. A witness graph in $\mathcal{A}_{\pi,(u, v)}$ with respect to a pair $(u, v)$ is a graph in which $(u, v)$ is part of a matched square $\left\langle u, v, u^{\prime}, v^{\prime}\right\rangle$. Potentially, 


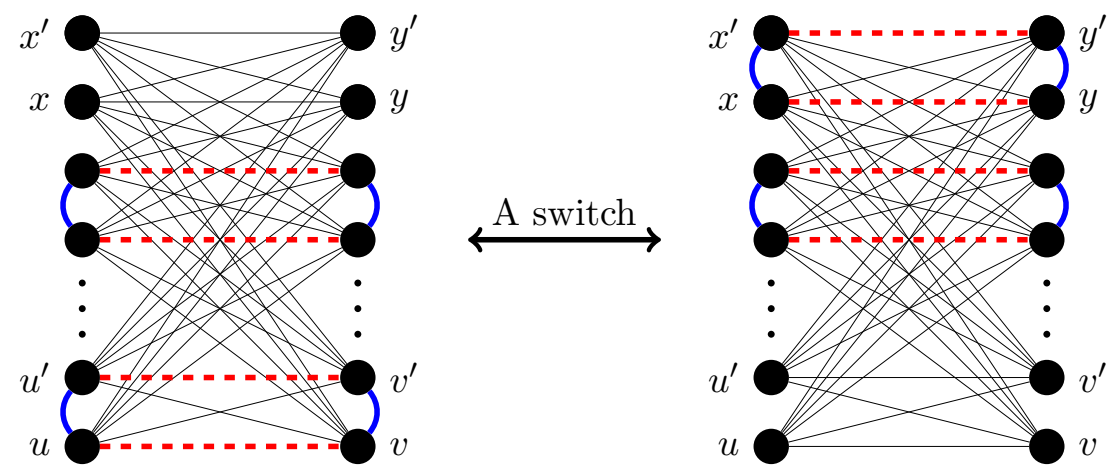

Figure 5: An illustration of a switch between the squares $\left\langle u, v, u^{\prime}, v^{\prime}\right\rangle$ and $\left\langle x, y, x^{\prime}, y^{\prime}\right\rangle$.

$\left\langle u, v, u^{\prime}, v^{\prime}\right\rangle$ could be switched with every unmatched square to get a non-witness pair. There are $\sqrt{m}-k$ unmatched vertices on each side, so that there are $\left(\begin{array}{c}\sqrt{m}-k \\ 2\end{array}\right) \cdot\left(\begin{array}{c}\sqrt{m}-k \\ 2\end{array}\right) \geq \frac{1}{8} m^{2}$ potential squares. To get a graph that is in $\mathcal{G}_{2}(\pi)$, the unmatched square $\left\langle x, y, x^{\prime}, y^{\prime}\right\rangle$ must be such that none of the induced pairs between the vertices $x, x^{\prime}, y, y^{\prime}$ have been observed yet by the algorithm. When all-neighbor queries are allowed, if at most $Q$ queries has been performed, then at most $4 Q$ pairs have been observed by the algorithm. Therefore, for at most $4 \frac{m}{100 k} \leq \frac{1}{4} m$ of the potential squares, an induced pair was queried. Hence, every witness square can be switched with at least $\frac{1}{8} m^{2}-\frac{1}{4} m \geq \frac{1}{16} m^{2}$ consistent unmatched squares, implying that $d_{w}\left(\mathcal{A}_{\pi,(u, v)}\right) \geq \frac{1}{16} m^{2}$.

To complete the proof it remains to show that $d_{n w}\left(\mathcal{A}_{\pi,(u, v)}\right) \leq m k$. To this end we would like to analyze the number of witness graphs that every non-witness $\bar{W}$ can be "turned" into. In every non-witness graph $\bar{W}$ the pair $(u, v)$ is unmatched, and in order to turn $\bar{W}$ into a witness graph, one of the $k$ matched squares should be removed and the pair $(u, v)$ with an additional pair $\left(u^{\prime}, v^{\prime}\right)$ should be "matched". There are $k$ options to remove an existing square, and at most $m$ options to choose a pair $u^{\prime}, v^{\prime}$ to match $(u, v)$ with. Therefore, the number of potential neighbors of $\bar{W}$ is at most $m k$. It follows that

$$
\frac{d_{n w}\left(\mathcal{A}_{\pi,(u, v)}\right)}{d_{w}\left(\mathcal{A}_{\pi,(u, v)}\right)}=\frac{16 m k}{m^{2}}=\frac{16 k}{m}=\frac{16 t}{m^{3 / 2}}
$$

and the proof is complete.

\subsubsection{Statistical distance}

For an all-neighbors query $q=u$ we say that the corresponding answer is a witness answer if $u \in L$ and $a \neq R$, or symmetrically if $u \in R$ and $a \neq L$. Let $E^{Q}$ be the set of all query-answer histories $\pi$ of length $Q$ such that there exists a query-answer pair $(q, a)$ in $\pi$ in which $q$ is an all-neighbors pair and $a$ is a witness answer with respect to that query, and let $\bar{E}^{Q}=\Pi^{Q} \backslash E^{Q}$. That is, $\bar{E}^{Q}$ is the set of all query-answer histories of length $Q$ such that no all-neighbors query is answered with a witness answer. Let $\widetilde{P}_{1}$ and $\widetilde{P}_{2}$ by the induced distributions of the processes $P_{1}$ and $P_{2}$ conditioned on the event that the process do not reply with a witness answer. Observe that for every query-answer history $\pi$ of length $t-1$, for every query $q_{t}$ that is either a pair query or a random new-neighbor query and for every $a \in A n s\left(\pi, q_{t}\right)$,

$$
\operatorname{Pr}_{\widetilde{P}_{b}}\left[a \mid \pi, q_{t}\right]=\operatorname{Pr}_{P_{b}}\left[a \mid \pi, q_{t}\right] .
$$

for $b \in\{1,2\}$. Therefore, the proof of the next lemma is exactly the same as the proof of Lemma 19, except that occurrences of the term $\left(t / m^{3 / 2}\right)$ are replaced by $(k / m)$ instead of $(1 / \sqrt{m})$ and we apply Lemma 24 instead of Lemma 18. 
Lemma 25. Let $t=k \cdot \sqrt{m}$ for an integer $k$ such that $1<k \leq \frac{\sqrt{m}}{4}$ and let $Q=\frac{m^{3 / 2}}{600 t}$. For every $t \leq Q$, every query-answer history $\pi$ of length $t-1$ such that $\pi$ is consistent with $G_{1}$ and for every pair or random new-neighbors query $q_{t}$,

$$
\sum_{a \in A n s\left(\pi, q_{t}\right)}\left|\operatorname{Pr}_{\widetilde{P}_{1}}\left[a \mid \pi, q_{t}\right]-\operatorname{Pr}_{\widetilde{P}_{2}}\left[a \mid \pi, q_{t}\right]\right|=\frac{96 k}{m}=\frac{96 t}{m^{3 / 2}} .
$$

Note that Lemma 25 does not cover all-neighbors queries, and hence we establish the next lemma.

Lemma 26. Let $t=k \cdot \sqrt{m}$ for an integer $k$ such that $1<k \leq \frac{\sqrt{m}}{4}$ and let $Q=\frac{m^{3 / 2}}{600 t}$. For every $t \leq Q$, every query-answer history $\pi$ of length $t-1$ such that $\pi$ is consistent with $G_{1}$ and for every all-neighbors query $q_{t}$,

$$
\operatorname{Pr}_{P_{2}}\left[a_{t} \text { is a witness answer } \mid \pi, q_{t}\right] \leq \frac{16 k}{\sqrt{m}} \text {. }
$$

Proof. Assume without loss of generality that $u \in L$. By the definition of the process $P_{2}$, it answers the query consistently with a uniformly selected random graph $G_{2} \in \mathcal{G}_{2}(\pi)$ by returning the complete set of $u$ 's neighbors in $G_{2}$. In $\mathcal{G}_{2}$, there are two types of graphs. First, there are graphs in which $u$ is not matched, that is $\left(u, u^{\prime}\right) \notin M^{L}$ for every vertex $u^{\prime} \in L$. In these graphs the set of $u$ 's neighbors is $R=\left\{r_{1}, \ldots, r_{\sqrt{m}}\right\}$. We refer to these graphs as non-witness graphs. The second type of graphs are those in which $\left(u, u^{\prime}\right) \in M^{L}$ for some $u^{\prime} \in L$ and $(u, v) \in M^{C}$ for some $v \in R$. In these graphs the set of $u$ 's neighbors is $(R \backslash\{v\}) \cup\left\{u^{\prime}\right\}$. We refer to these graphs as witness graphs. As before, let $A n s\left(\pi, q_{t}\right)$ be the set of all possible answers for an all-neighbors query $q_{t}$. It holds that

$$
\begin{aligned}
\operatorname{Pr}_{P_{2}}\left[a_{t} \text { is a witness answer } \mid \pi, q_{t}\right] & =\sum_{\substack{a \in \operatorname{Ans}\left(\pi, q_{t}\right) \\
a \neq R}} \operatorname{Pr}_{P_{2}}\left[a \mid \pi, q_{t}\right] \\
& =\sum_{u^{\prime} \in L, v \in R} \frac{\left|\mathcal{G}_{2}\left(\pi \circ\left(\left(u, u^{\prime}\right), 1\right) \circ((u, v), 0)\right)\right|}{\left|\mathcal{G}_{2}(\pi)\right|} \\
& =\sum_{u^{\prime} \in L} \frac{\left|\mathcal{G}_{2}\left(\pi \circ\left(\left(u, u^{\prime}\right), 1\right)\right)\right|}{\left|\mathcal{G}_{2}(\pi)\right|} \cdot \sum_{v \in R} \frac{\left|\mathcal{G}_{2}\left(\pi \circ\left(\left(u, u^{\prime}\right), 1\right) \circ((u, v), 0)\right)\right|}{\left|\mathcal{G}_{2}(\pi)\right|} \\
& =\sum_{u^{\prime} \in L} \frac{\left|\mathcal{G}_{2}\left(\pi \circ\left(\left(u, u^{\prime}\right), 1\right)\right)\right|}{\left|\mathcal{G}_{2}(\pi)\right|} .
\end{aligned}
$$

Similarly to the proof of Lemma 19, for every $u$ and $u^{\prime}$ in $L, \frac{\left|\mathcal{G}_{2}\left(\pi \circ\left(\left(u, u^{\prime}\right), 1\right)\right)\right|}{\left|\mathcal{G}_{2}(\pi)\right|} \leq \frac{16 k}{m}$. Therefore,

$$
\operatorname{Pr}_{P_{2}}\left[a_{t} \text { is a witness answer } \mid \pi, q_{t}\right]=\sum_{u^{\prime} \in L} \frac{\left|\mathcal{G}_{2}\left(\pi \circ\left(\left(u, u^{\prime}\right), 1\right)\right)\right|}{\left|\mathcal{G}_{2}(\pi)\right|} \leq \sqrt{m} \cdot \frac{16 k}{m}=\frac{16 k}{\sqrt{m}},
$$

and the lemma follows.

It remains to prove that a similar lemma to Lemma 20 holds for $\sqrt{m} \leq t \leq \frac{1}{4} m$ (and the distributions $\mathcal{D}_{1}^{\mathrm{ALG}}$ and $\mathcal{D}_{2}^{\mathrm{ALG}}$ as defined in this subsection).

Lemma 27. Let $t=k \cdot \sqrt{m}$ for an integer $k$ such that $1<k \leq \frac{\sqrt{m}}{4}$. For every algorithm ALG that performs at most $Q=\frac{m^{3 / 2}}{600 t}$ queries, the statistical distance between $\mathcal{D}_{1}^{A L G}$ and $\mathcal{D}_{2}^{A L G}$ is at most $\frac{1}{3}$. 
Proof. Let the sets $E^{Q}$ and $\bar{E}^{Q}$ be as defined in the beginning of this subsection. By the definition of the statistical distance, and since $\operatorname{Pr}_{P_{1}, \mathrm{ALG}}\left[E^{Q}\right]=0$,

$$
\begin{aligned}
d\left(\mathcal{D}_{1}^{\mathrm{ALG}}, \mathcal{D}_{2}^{\mathrm{ALG}}\right) & =\frac{1}{2}\left(\sum_{\pi \in E^{Q}}\left|\operatorname{Pr}_{P_{1}, \mathrm{ALG}}[\pi]-\operatorname{Pr}_{P_{2}, \mathrm{ALG}}[\pi]\right|+\sum_{\pi \in \bar{E}^{Q}}\left|\operatorname{Pr}_{P_{1}, \mathrm{ALG}}[\pi]-\operatorname{Pr}_{P_{2}, \mathrm{ALG}}[\pi]\right|\right) \\
& =\frac{1}{2}\left(\operatorname{Pr}_{P_{2}, \mathrm{ALG}}\left[E^{Q}\right]+\sum_{\pi \in \bar{E}^{Q}}\left|\operatorname{Pr}_{P_{1}, \mathrm{ALG}}[\pi]-\operatorname{Pr}_{P_{2}, \mathrm{ALG}}[\pi]\right|\right)
\end{aligned}
$$

By Lemma 26, the probability of detecting a witness as a result of an all-neighbors query is at most $\frac{16 k}{\sqrt{m}}$. Since in $Q$ queries, there can be at most $4 Q / \sqrt{m}$ all-neighbors queries, we have that

$$
\operatorname{Pr}_{\mathcal{D}_{2}^{\mathrm{ALG}}}\left[E^{Q}\right] \leq \frac{1}{6}
$$

We now turn to upper bound the second term. Let $\alpha=\operatorname{Pr}_{P_{2}, \mathrm{ALG}}\left[E^{Q}\right]$.

$$
\begin{aligned}
\sum_{\pi \in \bar{E}^{Q}}\left|\operatorname{Pr}_{P_{1}, \mathrm{ALG}}[\pi]-\operatorname{Pr}_{P_{2}, \mathrm{ALG}}[\pi]\right| & =\sum_{\pi \in \bar{E}^{Q}}\left|\operatorname{Pr}_{\widetilde{P}_{1}, \mathrm{ALG}}[\pi] \cdot \operatorname{Pr}_{P_{1}, \mathrm{ALG}}\left[\bar{E}^{Q}\right]-\operatorname{Pr}_{\widetilde{P}_{2}, \mathrm{ALG}}[\pi] \cdot \operatorname{Pr}_{P_{2}, \mathrm{ALG}}\left[\bar{E}^{Q}\right]\right| \\
& =\sum_{\pi \in \bar{E}^{Q}}\left|\operatorname{Pr}_{\widetilde{P}_{1}, \mathrm{ALG}}[\pi]-(1-\alpha) \cdot \operatorname{Pr}_{\widetilde{P}_{2}, \mathrm{ALG}}[\pi]\right| \\
& \leq \sum_{\pi \in \bar{E}^{Q}}\left|\operatorname{Pr}_{\widetilde{P}_{1}, \mathrm{ALG}}[\pi]-\operatorname{Pr}_{\widetilde{P}_{2}, \mathrm{ALG}}[\pi]\right|+\alpha \cdot \operatorname{Pr}_{\widetilde{P}_{2}, \mathrm{ALG}}\left[\bar{E}^{Q}\right] \\
& \leq \sum_{\pi \in \bar{E}^{Q}}\left|\operatorname{Pr}_{\widetilde{P}_{1}, \mathrm{ALG}}[\pi]-\operatorname{Pr}_{\widetilde{P}_{2}, \mathrm{ALG}}[\pi]\right|+\frac{1}{6}
\end{aligned}
$$

where in Equation (25) we used the fact that $\operatorname{Pr}_{P_{1}, \mathrm{ALG}}\left[\bar{E}^{Q}\right]=1$, and in Equation (26) we used the fact that $\operatorname{Pr}_{\widetilde{P}_{2}, \mathrm{ALG}}\left[\bar{E}^{Q}\right]=1$ and that $\alpha \leq 1 / 6$.

Therefore, it remains to bound

$$
\sum_{\pi \in \bar{E}^{Q}}\left|\operatorname{Pr}_{\widetilde{P}_{1}, \mathrm{ALG}}[\pi]-\operatorname{Pr}_{\widetilde{P}_{2}, \mathrm{ALG}}[\pi]\right| .
$$

Let the hybrid distributions $D_{1, t}^{\mathrm{ALG}}$ for $t \in[Q-1]$ be as defined in Lemma 20 (based on the distributions $\mathcal{D}_{1}^{\mathrm{ALG}}$ and $\mathcal{D}_{2}^{\mathrm{ALG}}$ that are induced by the processes $P_{1}$ and $P_{2}$ that were defined in this subsection). Also, let $\widetilde{\mathcal{D}}_{1, t}^{\mathrm{ALG}}$ be the hybrid distribution $\mathcal{D}_{1, t}^{\mathrm{ALG}}$ conditioned on the event that no all-neighbors query is answered with a witness. That is, $\widetilde{\mathcal{D}}_{1, t}^{\mathrm{ALG}}$ is the distribution over query-answer histories $\pi$ of length $Q$, where in the length $t$ prefix ALG is answered by the process $P_{1}$, in the length $Q-t$ suffix ALG is answered by the process $P_{2}$, and each all-neighbors query is answered consistently with $G_{1}$ (so that no witness is observed). By the above definitions and the triangle inequality,

$$
\sum_{\pi \in \bar{E}^{Q}}\left|\operatorname{Pr}_{\widetilde{P}_{1}, \mathrm{ALG}}[\pi]-\operatorname{Pr}_{\widetilde{P}_{2}, \mathrm{ALG}}[\pi]\right| \leq \sum_{t}^{Q-1} \sum_{\pi \in \bar{E}^{Q}}\left|\operatorname{Pr}_{\widetilde{\mathcal{D}}_{1, t+1}^{\mathrm{ALG}}}[\pi]-\operatorname{Pr}_{\widetilde{\mathcal{D}}_{1, t}^{\mathrm{ALG}}}[\pi]\right|
$$


As in the proof of Lemma 20 we have that for every $t \in[Q-1]$,

$$
\begin{aligned}
& \sum_{\pi \in \bar{E}^{Q}}\left|\operatorname{Pr}_{\widetilde{\mathcal{D}}_{1, t+1}^{\mathrm{ALG}}}[\pi]-\operatorname{Pr}_{\widetilde{\mathcal{D}}_{1, t}^{\mathrm{ALG}}}[\pi]\right| \\
& =\sum_{\substack{\pi^{\prime}=\pi_{1}, \ldots, \pi_{t-1}, q_{t}: \\
\pi^{\prime} \in \bar{E}^{t-1}}} \operatorname{Pr}_{\widetilde{P}_{1}, \mathrm{ALG}}\left[\pi^{\prime}, q_{t}\right] \cdot \sum_{\substack{a \in A n s\left(\pi^{\prime}, q_{t}\right): \\
\pi^{\prime} \circ\left(q_{t}, a\right) \in \bar{E}^{t}}}\left|\operatorname{Pr}_{\widetilde{P}_{1}}\left[a \mid \pi^{\prime}, q_{t}\right]-\operatorname{Pr}_{\widetilde{P}_{2}}\left[a \mid \pi^{\prime}, q_{t}\right]\right| .
\end{aligned}
$$

By Lemma 25 (and since for an all-neighbor query $q_{t}$ we have that the (unique) answer according to $\widetilde{P}_{2}$ is the same as according to $\widetilde{P}_{1}$ ),

$$
\sum_{\substack{a \in A n s\left(\pi^{\prime}, q_{t}\right): \\ \pi^{\prime} \circ\left(q_{t}, a\right) \in \bar{E}^{t}}}\left|\operatorname{Pr}_{\widetilde{P}_{1}}\left[a \mid \pi^{\prime}, q_{t}\right]-\operatorname{Pr}_{\widetilde{P}_{2}}\left[a \mid \pi^{\prime}, q_{t}\right]\right| \leq \frac{96 k}{m}=\frac{96 t}{m^{3 / 2}},
$$

and it follows that

$$
\sum_{\pi \in \bar{E}^{Q}}\left|\operatorname{Pr}_{\widetilde{\mathcal{D}}_{1, t+1}^{\mathrm{ALG}}}[\pi]-\operatorname{Pr}_{\widetilde{\mathcal{D}}_{1, t}^{\mathrm{ALG}}}[\pi]\right| \leq \frac{96 k}{m}=\frac{96 t}{m^{3 / 2}}
$$

Hence, for $Q=\frac{m^{3 / 2}}{600 t}$,

$$
\sum_{t}^{Q-1} \sum_{\pi \in \bar{E}^{Q}}\left|\operatorname{Pr}_{\widetilde{\mathcal{D}}_{1, t+1}^{\mathrm{ALG}}}[\pi]-\operatorname{Pr}_{\widetilde{\mathcal{D}}_{1, t}^{\mathrm{ALG}}}[\pi]\right| \leq Q \cdot \frac{48 t}{m^{3 / 2}} \leq \frac{1}{6}
$$

Combining Equations (23), (24), (26), (27) and (29), we get

$$
d\left(\mathcal{D}_{1}^{\mathrm{ALG}}, \mathcal{D}_{2}^{\mathrm{ALG}}\right) \leq \frac{1}{2}\left(\frac{1}{6}+\frac{1}{6}+\frac{1}{6}\right) \leq \frac{1}{3}
$$

and the proof is complete.

\subsection{Lower Bound for $t<\frac{1}{4} \sqrt{m}$.}

\subsubsection{The construction}

In this case the basic structure of $G_{1}$ and $\mathcal{G}_{2}$ is a bit different. Also, for the sake of simplicity, we present graphs with $2 m$ edges, and either 0 or $4 t$ triangles. The graph $G_{1}$ has three components two complete bipartite graphs, each over $2 \sqrt{m}$ vertices, and an independent set of size $n-4 \sqrt{m}$. Let $A$ and $B$ be the left-hand side and the right-hand side sets, respectively, of the first bipartite component, and $C$ and $D$ of the second one. We refer to the edges between $A$ and $B$ and the edges between $C$ and $D$ as black edges. We divide each of these sets into $\frac{\sqrt{m}}{t}$ subsets of size $t$, denoted $\left\{\Lambda_{1}, \ldots, \Lambda_{\frac{\sqrt{m}}{t}}\right\}$ for $\Lambda \in\{A, B, C, D\}$. For every $1 \leq i \leq \frac{\sqrt{m}}{t}$, we first remove a complete bipartite graph between $A_{i}$ and $B_{i}$ and between $C_{i}$ and $D_{i}$, and refer to the removed edges as red edges. We then add a complete bipartite graph between $B_{i}$ and $C_{i}$ and between $D_{i}$ and $A_{i}$, and refer to added edges as blue edges. Note that this maintains the degrees of all the vertices to be $\sqrt{m}$.

In $\mathcal{G}_{2}$ the basic structure of all the graphs is the same as of $G_{1}$ with the following modifications. Each graph is defined by the choice of four "special" vertices $a^{*}, b^{*}, c^{*}, d^{*}$ such that $a^{*} \in A_{i_{a^{*}}}, b^{*} \in$ $B_{i_{b^{*}}}, c^{*} \in C_{i_{c^{*}}}$ and $d^{*} \in D_{i_{d^{*}}}$ for some indices $i_{a^{*}}, i_{b^{*}}, i_{c^{*}}$ and $i_{d^{*}}$ such that no two indices are equal. We then add edges $\left(a^{*}, c^{*}\right)$ and $\left(b^{*}, d^{*}\right)$, referred to as green edges, and remove edges $\left(a^{*}, b^{*}\right)$ and 


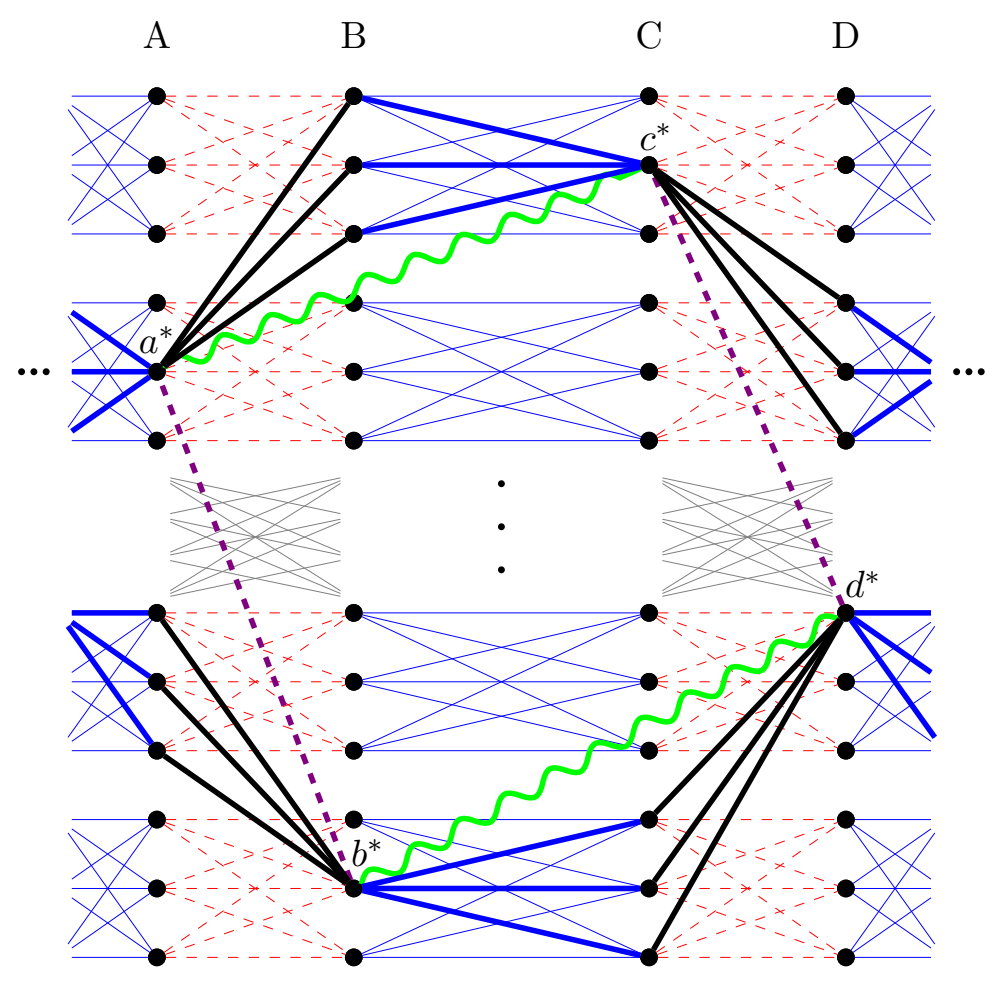

Figure 6: An illustration of a graph in $\mathcal{G}_{2}$. The broken thin (red) edges describe edges that were removed and the thin (blue) edges describe edges that were added. The broken thick (purple) edges describe the special non-edges $\left(a^{*}, b^{*}\right)$ and $\left(c^{*}, d^{*}\right)$. The curly (green) edges describe the special edges $\left(a^{*}, c^{*}\right)$ and $\left(b^{*}, d^{*}\right)$.

$\left(c^{*}, d^{*}\right)$, referred to as purple edges. We also refer to the green and purple edges as special edges. Note that we add one edge and remove one edge from each special vertex, thus maintaining their initial degrees. See Figure 6.

We first prove that $t\left(G_{1}\right)=0$ and then that for every graph $G$ in $\mathcal{G}_{2}, t(G)=4 t$.

Claim 28. The graph $G_{1}$ has no triangles.

Proof. Consider an edge $(u, v)$ in $G_{1}$. First assume $u$ and $v$ are connected by a black edge, that is, they are on different sides of the same bipartite component. Hence we can assume without loss of generality that $u \in A$ and that $v \in B$. Since $u$ is in $A$ it is only connected to vertices in $B$ or vertices in $D$. Since $v$ is in $B$ it is only connected to vertices in $A$ or vertices in $C$. Thus $u$ and $v$ cannot have a common neighbor. A similar analysis can be done for a pair $(u, v)$ that is connected by a blue edge. Therefore $t(G)$ is indeed zero as claimed.

Claim 29. For every graph $G \in \mathcal{G}_{2}, t(G)=4 t$.

Proof. Since the only differences between $G_{1}$ and graphs in $\mathcal{G}_{2}$ are the two added green edges and the two removed red edges, any triangle in $\mathcal{G}_{2}$ must include a green edge. Therefore we can count all the triangles that the green edges form. Consider the green edge $\left(a^{*}, c^{*}\right)$ and recall that $a^{*}$ is in $A_{i_{a^{*}}}$ and $c^{*}$ is in $C_{i_{c^{*}}}$. The only common neighbors of $\left(a^{*}, c^{*}\right)$ are all the vertices in $B_{i_{c^{*}}}$ and all the vertices in $D_{i_{a^{*}}}$. A vertex $v$ such that $v \notin B_{i_{c^{*}}}$ and $v \notin D_{i_{a^{*}}}$ is either (1) in $A$ or in $D \backslash D_{i_{a^{*}}}$, in which case it is not a neighbor of $a^{*}$, or it is (2) in $C$ or in $B \backslash B_{i_{c^{*}}}$, in which case it is not a neighbor 
of $c^{*}$. Since both $B_{i_{c^{*}}}$ and $D_{i_{a^{*}}}$ are of size $t$, the edge $\left(a^{*}, c^{*}\right)$ participates in $2 t$ triangles. Similarly the edge $\left(b^{*}, d^{*}\right)$ participate in $2 t$ triangles, and together we get that $t(G)=4 t$, as claimed.

\subsubsection{The processes $P_{1}$ and $P_{2}$}

The definition of the processes $P_{1}$ and $P_{2}$ is the same as in Subsection 4.3.2 (using the modified definitions of $G_{1}$ and $\mathcal{G}_{2}$ ).

\subsubsection{The auxiliary graph}

We define a switch for this case as well. Informally, a switch between a matched pair $\left(u^{*}, v^{*}\right)$ and an unmatched pair $(u, v)$ is "unmatching" $\left(u^{*}, v^{*}\right)$ and "matching" $(u, v)$ instead. Formally stating we define a switch as follows.

Definition 9. A switch between a green pair $\left(a^{*}, c^{*}\right)$ and a pair $(a, c)$ such that $a \in A_{i}, c \in C_{j}$ and none of the indices $i, j, i_{b^{*}}, i_{d^{*}}$ are equal, is the following two steps process. In the first step we "unmatch" $\left(a^{*}, c^{*}\right)$ by removing the green edge $\left(a^{*}, c^{*}\right)$ and adding the edges $\left(a^{*}, b^{*}\right)$ and $\left(c^{*}, d^{*}\right)$. In the second step we "match" $(a, c)$ by adding the green edge $(a, c)$ and removing the edges $\left(a, b^{*}\right)$ and $\left(c, d^{*}\right)$. A switch with the pair $\left(b^{*}, d^{*}\right)$ can be defined in a similar manner.

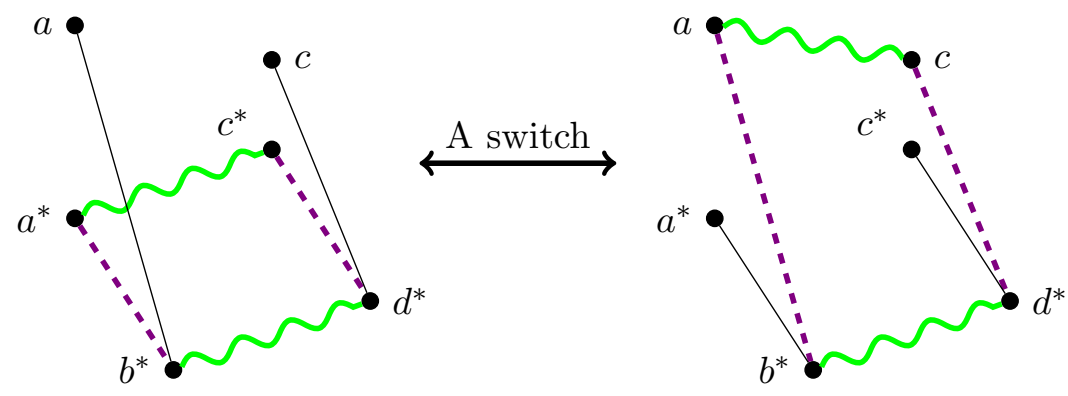

Figure 7: An illustration of a switch between the pairs $\left(a^{*}, c^{*}\right)$ and $(a, c)$.

Let $t<\sqrt{m}$ and let $Q=\frac{m}{600}$. For every $t \leq Q$, every query-answer history $\pi$ of length $t-1$ and every pair $(u, v)$ we define the following auxiliary graph. The witness nodes are graphs in which $(u, v)$ is one of the four special pairs. If the pair is a green matched pair then there is an edge in the auxiliary graph between a witness graph $W$ and a non-witness graph $\bar{W}$, if $\bar{W}$ can be obtained from $W$ by a single switch between $(u, v)$ and another unmatched pair.

Lemma 30. For $t<\frac{1}{4} \sqrt{m}$ let $Q=\frac{m}{600}$. For every $t \leq Q$, every query-answer history $\pi$ of length $t-1$ such that $\pi$ is consistent with $G_{1}$ and every pair $(u, v)$,

$$
\frac{d_{n w}\left(\mathcal{A}_{\pi,(u, v)}\right)}{d_{w}\left(\mathcal{A}_{\pi,(u, v)}\right)}=\frac{8}{m}
$$

Proof. We analyze the case where the pair $(u, v)$ is such that $u \in A$ and $v \in C$, as the proof for the other cases is almost identical. We first prove that $d_{w}\left(\mathcal{A}_{\pi,(u, v)}\right) \geq \frac{1}{8} m$. A witness graph $W$ is a graph in which $(a, c)$ is a special pair. That is $(u, v)=\left(a^{*}, c^{*}\right)$. Potentially, for every pair $\left(a^{\prime}, c^{\prime}\right)$ such that $a^{\prime} \in A_{i}, c^{\prime} \in C_{j}$ and none of the indices $i, j, i_{b^{*}}, i_{d^{*}}$ are equal, the graph resulting from a switch between $\left(a^{*}, c^{*}\right)$ and $\left(a^{\prime}, c^{\prime}\right)$ is a non-witness graph. There are $\sqrt{m}-2 t$ vertices $a^{\prime}$ in $A \backslash\left(A_{i_{b^{*}}} \cup A_{i_{d^{*}}}\right)$ and for each such $a^{\prime}$ there are $\sqrt{m}-3 t$ vertices $c^{\prime}$ in $\left.C \backslash\left(C_{i_{b^{*}}} \cup C_{i_{d^{*}}} \cup C_{i_{a^{\prime}}}\right)\right)$. Since 
$t<\frac{1}{4} \sqrt{m}$, there are at least $(\sqrt{m}-2 t) \cdot(\sqrt{m}-3 t)=m-6 t^{2} \geq \frac{1}{4} m$ potential pairs $\left(a^{\prime}, c^{\prime}\right)$ that $\left(a^{*}, c^{*}\right)$ could be switched with. For the resulting graph to be consistent, that is, to be in $\mathcal{G}_{2}(\pi)$, the pair $\left(a^{\prime}, c^{\prime}\right)$ must be such that the pairs $\left(a^{\prime}, c^{\prime}\right),\left(a^{*}, b^{*}\right)$ and $\left(c^{*}, d^{*}\right)$ have not been observed yet by the algorithm. Since the number of queries is at most $\frac{1}{600} m$, at least $\frac{1}{4} m-\frac{1}{125} m \geq \frac{1}{8} m$ of the potential pairs $\left(a^{\prime}, c^{\prime}\right)$ can be switched with $\left(a^{*}, c^{*}\right)$ such that the resulting graph is consistent with $\mathcal{G}_{2}(\pi)$. Therefore, $d_{w}\left(\mathcal{A}_{\pi,(u, v)}\right) \geq \frac{1}{8} m$.

Now consider a non-witness graph $\bar{W}$. There is only one possibility to turn $\bar{W}$ into a witness graph, which is to switch the pair $(u, v)$ with the green pair $\left(a^{*}, c^{*}\right)$. Therefore, the maximal degree of every non-witness graph, $d_{n w}\left(\mathcal{A}_{\pi,(u, v)}\right)$, is 1 .

Together we get that

$$
\frac{d_{n w}\left(\mathcal{A}_{\pi,(u, v)}\right)}{d_{w}\left(\mathcal{A}_{\pi,(u, v)}\right)} \leq \frac{8}{m}
$$

and the proof is complete.

\subsubsection{Statistical distance}

A similar proof to the ones of Lemma 25 and Lemma 26 using Lemma 30 gives the following lemmas for the case that $1 \leq t<\frac{1}{4} \sqrt{m}$.

Lemma 31. Let $1 \leq t<\frac{1}{4} \sqrt{m}$ and $Q=\frac{m}{600}$. For every $t \leq Q$, every query-answer history $\pi$ of length $t-1$ such that $\pi$ is consistent with $G_{1}$ and for every all-neighbors query $q_{t}$,

$$
\operatorname{Pr}_{P_{2}}\left[a_{t} \text { is a witness answer } \mid \pi, q_{t}\right] \leq \frac{16}{m} .
$$

Lemma 32. Let $1 \leq t<\frac{1}{4} \sqrt{m}$ and $Q=\frac{m}{600}$. For every $t \leq Q$, every query-answer history $\pi$ of length $t-1$ such that $\pi$ is consistent with $G_{1}$ and for every pair or random new-neighbors query $q_{t}$,

$$
\sum_{a \in \operatorname{Ans}\left(\pi, q_{t}\right)}\left|\operatorname{Pr}_{\widetilde{P}_{1}}\left[a \mid \pi, q_{t}\right]-\operatorname{Pr}_{\widetilde{P}_{2}}\left[a \mid \pi, q_{t}\right]\right|=\frac{96}{m} .
$$

The next lemma is proven in a similar way to 1.3.4 based on the above two lemma.

Lemma 33. Let $1 \leq t<\frac{1}{4} \sqrt{m}$. For every algorithm $A L G$ that asks at most $Q=\frac{m}{600}$, the statistical distance between $\mathcal{D}_{1}^{A L G}$ and $\mathcal{D}_{2}^{A L G}$ is at most $\frac{1}{3}$.

\subsection{Wrapping things up}

Theorem 15 follows from Lemmas 20,23, 27 and 33, and the next corollary is proved using Theorems 15 and 14.

Corollary 34. Any multiplicative-approximation algorithm for the number of triangles in a graph must perform $\Omega\left(\frac{n}{t(G)^{1 / 3}}+\min \left\{m, \frac{m^{3 / 2}}{t(G)}\right\}\right)$ queries, where the allowed queries are degree queries, pair queries and neighbor queries.

Proof. Assume towards a contradiction that there exists an algorithm ALG' for which the following holds:

1. ALG' is allowed to ask neighbor queries as well as degree queries and pair queries.

2. ALG' asks $Q^{\prime}$ queries. 
3. ALG' outputs a $(1 \pm \epsilon)$-approximation to the number of triangles of any graph $G$ with probability greater than $2 / 3$.

Using ALG' we can define an algorithm ALG that is allowed random new-neighbor queries, performs at most $Q=3 Q^{\prime}$ queries and answers correctly with the same probability as ALG' does. ALG runs ALG' and whenever ALG' performs a query $q_{t}^{\prime}$, ALG does as follows:

- If $q_{t}^{\prime}$ is a degree query, ALG performs the same query and sets $a_{t}^{\prime}=a_{t}$.

- If $q_{t}^{\prime}$ is a pair query $(u, v)$, then ALG performs the same query $q=q^{\prime}$. Let $a_{t}$ be the corresponding answer.

- If $a_{t}=0$, then ALG sets $a_{t}^{\prime}=a_{t}$.

- If $a_{t}=1$, then ALG sets $a_{t}^{\prime}=\left(a_{t}, i, j\right)$, such that $i$ and $j$ are randomly chosen labels that have not been previously used for neighbors of $u$ and $v$, and are within the ranges $[1 . . d(u)]$ and $\left[1 . . d_{v}\right]$ respectively.

- If $q_{t}^{\prime}$ is a neighbor query $(u, i)$, ALG performs a random new-neighbor query $q_{t}=u$, and returns the same answer $a_{t}^{\prime}=a_{t}$.

We note that the above requires the algorithm ALG to store for every vertex $v$, all the labels used for its neighbors in the previous steps. Once ALG' outputs an answer, ALG outputs the same answer. It follows that ALG performs at most $3 Q$ queries to the graph $G$. By the third assumption above, ALG outputs a $(1 \pm \epsilon)$-approximation to the number of triangles of any graph $G$ with probability greater than $2 / 3$. If $Q^{\prime} \notin \Omega\left(\frac{n}{t(G)^{1 / 3}}+\min \left\{m, \frac{m^{3 / 2}}{t(G)}\right\}\right)$ then $Q \notin \Omega\left(\frac{n}{t(G)^{1 / 3}}+\min \left\{m, \frac{m^{3 / 2}}{t(G)}\right\}\right)$ which is a contradiction to Theorem 14 and Theorem 15.

\section{References}

[ADNK14] N. K. Ahmed, N. Duffield, J. Neville, and R. Kompella. Graph sample and hold: A framework for big graph analytics. In Conference on Knowledge Discovery and Data Mining (KDD), 2014. 2, 6

[AGM12] K. J. Ahn, S. Guha, and A. McGregor. Graph sketches: sparsification, spanners, and subgraphs. In Principles of Database Systems, pages 5-14, 2012. 2, 6

[AKM13] Shaikh Arifuzzaman, Maleq Khan, and Madhav Marathe. Patric: A parallel algorithm for counting triangles in massive networks. In Proceedings of the 22nd ACM international conference on Conference on information $\&$ knowledge management, pages 529-538. ACM, 2013. 2, 6

[Avr10] Haim Avron. Counting triangles in large graphs using randomized matrix trace estimation. In Workshop on Large-scale Data Mining: Theory and Applications, volume 1, 2010. 2, 6

[AYZ97] Noga Alon, Raphael Yuster, and Uri Zwick. Finding and counting given length cycles. Algorithmica, 17(3):209-223, 1997. 2, 5

[BBCG08] Luca Becchetti, Paolo Boldi, Carlos Castillo, and Aristides Gionis. Efficient semistreaming algorithms for local triangle counting in massive graphs. In Proceedings of the 14th ACM SIGKDD international conference on Knowledge discovery and data mining, pages 16-24. ACM, 2008. 2, 6 
$\left[\mathrm{BFL}^{+}\right.$06] Luciana S Buriol, Gereon Frahling, Stefano Leonardi, Alberto Marchetti-Spaccamela, and Christian Sohler. Counting triangles in data streams. In Proceedings of the twenty-fifth ACM SIGMOD-SIGACT-SIGART symposium on Principles of database systems, pages 253-262. ACM, 2006. 2, 6

[BHLP11] J. W. Berry, B. Hendrickson, R. A. LaViolette, and C. A. Phillips. Tolerating the Community Detection Resolution Limit with Edge Weighting. Physical Review E, 83(5), May 2011. 2

[BPWZ14] A. Björklund, R. Pagh, V. Vassilevska Williams, and U. Zwick. Listing triangles. In Proceedings of International Colloquium on Automata, Languages, and Programming (ICALP), pages 223-234, 2014. 2, 5

[Bur04] R. S. Burt. Structural holes and good ideas. American Journal of Sociology, 110(2):349-399, 2004. 2

[BYKS02] Ziv Bar-Yossef, Ravi Kumar, and D Sivakumar. Reductions in streaming algorithms, with an application to counting triangles in graphs. In Proceedings of the Thirteenth Annual ACM-SIAM Symposium on Discrete Algorithms (SODA), pages 623-632. Society for Industrial and Applied Mathematics, 2002. 2, 6

[CC11] Shumo Chu and James Cheng. Triangle listing in massive networks and its applications. In Proceedings of the 17th ACM SIGKDD international conference on Knowledge discovery and data mining, pages 672-680. ACM, 2011. 2, 6

$\left[\mathrm{CEF}^{+} 05\right]$ Artur Czumaj, Funda Ergün, Lance Fortnow, Avner Magen, Ilan Newman, Ronitt Rubinfeld, and Christian Sohler. Approximating the weight of the euclidean minimum spanning tree in sublinear time. SIAM Journal on Computing, 35(1):91-109, 2005. 5

[CN85] Norishige Chiba and Takao Nishizeki. Arboricity and subgraph listing algorithms. SIAM Journal on Computing, 14(1):210-223, 1985. 2, 5, 11

[Coh09] J. Cohen. Graph twiddling in a MapReduce world. Computing in Science \& Engineering, 11:29-41, 2009. 6

[Col88] J. S. Coleman. Social capital in the creation of human capital. American Journal of Sociology, 94:S95-S120, 1988. 2

[CRT05] Bernard Chazelle, Ronitt Rubinfeld, and Luca Trevisan. Approximating the minimum spanning tree weight in sublinear time. SIAM Journal on Computing, 34(6):13701379, 2005. 5

[CS09] Artur Czumaj and Christian Sohler. Estimating the weight of metric minimum spanning trees in sublinear time. SIAM Journal on Computing, 39(3):904-922, 2009. 5

[EM02] J.-P. Eckmann and E. Moses. Curvature of co-links uncovers hidden thematic layers in the World Wide Web. Proceedings of the National Academy of Sciences (PNAS), 99(9):5825-5829, 2002. 2

[Fei06] Uriel Feige. On sums of independent random variables with unbounded variance and estimating the average degree in a graph. SIAM Journal on Computing, 35(4):964$984,2006.3,5,7,11,15,16$ 
[FWVDC10] Brooke Foucault Welles, Anne Van Devender, and Noshir Contractor. Is a friend a friend?: Investigating the structure of friendship networks in virtual worlds. In CHI'10 Extended Abstracts on Human Factors in Computing Systems, pages 40274032. ACM, 2010. 2

[GR02] Oded Goldreich and Dana Ron. Property testing in bounded degree graphs. Algorithmica, pages 302-343, 2002. 19

[GR08] Oded Goldreich and Dana Ron. Approximating average parameters of graphs. Random Structures and Algorithms, 32(4):473-493, 2008. 3, 5

[GRS11] Mira Gonen, Dana Ron, and Yuval Shavitt. Counting stars and other small subgraphs in sublinear-time. SIAM Journal on Discrete Math, 25(3):1365-1411, 2011. 2, 5

[HKNO09] Avinatan Hassidim, Jonathan A Kelner, Huy N Nguyen, and Krzysztof Onak. Local graph partitions for approximation and testing. In Proceedings of the Fiftieth Annual Symposium on Foundations of Computer Science (FOCS), pages 22-31. IEEE, 2009. 5

[HL70] P. W. Holland and S. Leinhardt. A method for detecting structure in sociometric data. American Journal of Sociology, 76:492-513, 1970. 2

[IR78] Alon Itai and Michael Rodeh. Finding a minimum circuit in a graph. SIAM Journal on Computing, 7(4):413-423, 1978. 2, 5

[JG05] Hossein Jowhari and Mohammad Ghodsi. New streaming algorithms for counting triangles in graphs. In Computing and Combinatorics, pages 710-716. Springer, 2005. 2,6

[JSP13] M. Jha, C. Seshadhri, and A. Pinar. A space efficient streaming algorithm for triangle counting using the birthday paradox. In Proceedings of the 19th ACM SIGKDD international conference on Knowledge discovery and data mining, KDD '13, pages 589-597, New York, NY, USA, 2013. ACM. 2, 6

[KMPT12] Mihail N Kolountzakis, Gary L Miller, Richard Peng, and Charalampos E Tsourakakis. Efficient triangle counting in large graphs via degree-based vertex partitioning. Internet Mathematics, 8(1-2):161-185, 2012. 2, 6

[KMSS12] D. M. Kane, K. Mehlhorn, T. Sauerwald, and H. Sun. Counting arbitrary subgraphs in data streams. In International Colloquium on Automata, Languages, and Programming (ICALP), pages 598-609, 2012. 2, 6

$\left[\mathrm{KPP}^{+} 13\right] \quad$ Tamara G. Kolda, Ali Pinar, Todd Plantenga, C. Seshadhri, and Christine Task. Counting triangles in massive graphs with MapReduce. arXiv:1301.5887, January 2013. 6

[MR09] Sharon Marko and Dana Ron. Approximating the distance to properties in boundeddegree and general sparse graphs. ACM Transactions on Algorithms, 5(2), 2009. 5

[MSOI $\left.{ }^{+} 02\right]$ Ron Milo, Shai Shen-Orr, Shalev Itzkovitz, Nadav Kashtan, Dmitri Chklovskii, and Uri Alon. Network motifs: simple building blocks of complex networks. Science, 298(5594):824-827, 2002. 2 
[NO08] Huy N Nguyen and Krzysztof Onak. Constant-time approximation algorithms via local improvements. In Proceedings of the Forty-Ninth Annual Symposium on Foundations of Computer Science (FOCS), pages 327-336. IEEE, 2008. 5

[ORRR12] Krzysztof Onak, Dana Ron, Michal Rosen, and Ronitt Rubinfeld. A near-optimal sublinear-time algorithm for approximating the minimum vertex cover size. In Proceedings of the Twenty-Third Annual ACM-SIAM Symposium on Discrete Algorithms (SODA), pages 1123-1131. SIAM, 2012. 5

[Por00] Alejandro Portes. Social capital: Its origins and applications in modern sociology. LESSER, Eric L. Knowledge and Social Capital. Boston: Butterworth-Heinemann, pages $43-67,2000.2$

[PR07] Michal Parnas and Dana Ron. Approximating the minimum vertex cover in sublinear time and a connection to distributed algorithms. Theoretical Computer Science, 381(1-3):183-196, 2007. 5

[PT12] R. Pagh and C. Tsourakakis. Colorful triangle counting and a mapreduce implementation. Information Processing Letters, 112:277-281, 2012. 6

[PTTW13] A. Pavan, K. Tangwongsan, S. Tirthapura, and K.-L. Wu. Counting and sampling triangles from a graph stream. In International Conference on Very Large Databases (VLDB), 2013. 2, 6

[SKP12] C. Seshadhri, T. G. Kolda, and A. Pinar. Community structure and scale-free collections of Erdös-Rényi graphs. Physical Review E, 85(5):056109, May 2012. 2

[SPK13] C. Seshadhri, A. Pinar, and T. G. Kolda. Fast triangle counting through wedge sampling. In Proceedings of the SIAM Conference on Data Mining, 2013. 2, 6

[SV11] S. Suri and S. Vassilvitskii. Counting triangles and the curse of the last reducer. In World Wide Web (WWW), pages 607-614, 2011. 2, 6

[SW05a] T. Schank and D. Wagner. Approximating clustering coefficient and transitivity. Journal of Graph Algorithms and Applications, 9:265-275, 2005. 2

[SW05b] T. Schank and D. Wagner. Finding, counting and listing all triangles in large graphs, an experimental study. In Experimental and Efficient Algorithms, pages 606-609. Springer Berlin / Heidelberg, 2005. 2, 6

$\left[\mathrm{TDM}^{+} 09\right]$ Charalampos E Tsourakakis, Petros Drineas, Eirinaios Michelakis, Ioannis Koutis, and Christos Faloutsos. Spectral counting of triangles in power-law networks via element-wise sparsification. In Social Network Analysis and Mining, 2009. ASONAM'09. International Conference on Advances in, pages 66-71. IEEE, 2009. 6

[TKM11] Charalampos E Tsourakakis, Mihail N Kolountzakis, and Gary L Miller. Triangle sparsifiers. J. Graph Algorithms Appl., 15(6):703-726, 2011. 2, 6

[TKMF09] Charalampos E Tsourakakis, U Kang, Gary L Miller, and Christos Faloutsos. Doulion: counting triangles in massive graphs with a coin. In Proceedings of the 15th ACM SIGKDD international conference on Knowledge discovery and data mining, pages 837-846. ACM, 2009. 2 
[TPT13] K. Tangwongsan, A. Pavan, and S. Tirthapura. Parallel triangle counting in massive streaming graphs. In ACM Conference on Information $\&$ Knowledge Management (CIKM), 2013. 2, 6

[Tso08] Charalampos E Tsourakakis. Fast counting of triangles in large real networks without counting: Algorithms and laws. In Data Mining, 2008. ICDM'08. Eighth IEEE International Conference on, pages 608-617. IEEE, 2008. 2, 6

[YYI09] Yuichi Yoshida, Masaki Yamamoto, and Hiro Ito. An improved constant-time approximation algorithm for maximum. In Proceedings of the Fourty-First Annual ACM Symposium on the Theory of Computing, pages 225-234. ACM, 2009. 5 